\title{
High-dimensional asset pricing and portfolio optimization
}

Citation for published version (APA):

Lönn, R. (2019). High-dimensional asset pricing and portfolio optimization. [Doctoral Thesis, Maastricht University]. Universitaire Pers Maastricht. https://doi.org/10.26481/dis.20191031sl

Document status and date:

Published: 01/01/2019

DOI:

10.26481/dis.20191031sl

Document Version:

Publisher's PDF, also known as Version of record

\section{Please check the document version of this publication:}

- A submitted manuscript is the version of the article upon submission and before peer-review. There can be important differences between the submitted version and the official published version of record.

People interested in the research are advised to contact the author for the final version of the publication, or visit the DOI to the publisher's website.

- The final author version and the galley proof are versions of the publication after peer review.

- The final published version features the final layout of the paper including the volume, issue and page numbers.

Link to publication

\footnotetext{
General rights rights.

- You may freely distribute the URL identifying the publication in the public portal. please follow below link for the End User Agreement:

www.umlib.nl/taverne-license

Take down policy

If you believe that this document breaches copyright please contact us at:

repository@maastrichtuniversity.nl

providing details and we will investigate your claim.
}

Copyright and moral rights for the publications made accessible in the public portal are retained by the authors and/or other copyright owners and it is a condition of accessing publications that users recognise and abide by the legal requirements associated with these

- Users may download and print one copy of any publication from the public portal for the purpose of private study or research.

- You may not further distribute the material or use it for any profit-making activity or commercial gain

If the publication is distributed under the terms of Article $25 \mathrm{fa}$ of the Dutch Copyright Act, indicated by the "Taverne" license above, 


\section{High-dimensional asset pricing and portfolio optimization}

Sven Olof Rasmus Lönn 
(C) Sven Olof Rasmus Lönn, Maastricht 2019

All rights reserved. No part of this publication may be reproduced, stored in a retrieval system, or transmitted in any form, or by any means, electronic, mechanical, photocopying, recording or otherwise, without the prior permission in writing from the author.

The cover is based on artwork provided by Marianne Lönn.

This book was typeset by the author using $\mathrm{LT}_{\mathrm{E}} \mathrm{X}$.

Published by Universitaire Pers Maastricht

ISBN: 978-94-6380-531-5

Printed in The Netherlands by ProefschriftMaken Maastricht 


\title{
High-dimensional asset pricing and portfolio optimization
}

\author{
Dissertation \\ to obtain the degree of Doctor at Maastricht University, \\ on the authority of the Rector Magnificus, \\ Prof.dr. Rianne M. Letschert, \\ in accordance with the decision of the Board of Deans, \\ to be defended in public \\ on Thursday, 31 October 2019, at 12.00 o'clock
}

by

Sven Olof Rasmus Lönn 


\section{Supervisor}

Prof.dr. P.C. Schotman

\section{Co-supervisors}

Dr. N. Bastürk

Dr. L. Lieb

\section{Assessment Committee}

Prof.dr. J.W.B. Bos (Chair)

Dr. S.J.M. Smeekes

Prof.dr. F.R. Kleibergen (University of Amsterdam)

Prof.dr. M. Lambert (University of Liége) 
In memory of my grandfather, Sven Olov Karlsson 



\section{Acknowledgements}

"Nothing in this world can take the place of persistence. Talent will not: nothing is more common than unsuccessful men with talent. Genius will not; unrewarded genius is almost a proverb. Education will not: the world is full of educated derelicts. Persistence and determination alone are omnipotent."

- Calvin Coolidge (1872-1933) 
During my first course of macroeconomics in Gothenburg I was thinking of how fantastic it must be to pursue a Ph.D. in Economics. Indeed, it took many years before I encountered a topic that I did not enjoy in this field. But without a doubt, my favorite material was always the empirically driven economics and econometrics. In retrospect, it also turned out to be a great time for empirical economics and finance, times of crises and turbulence, and a great number of excellent anomalies still remain. If this is the best of all possible worlds then economic research seems like an enduring pursuit. As this first part of my pursuit is coming to an end there are many friends, family and colleagues that I want to thank and whose contributions I want to acknowledge.

My time as a Ph.D. student has been profoundly formative for me. This is mostly thanks to my supervisors Peter Schotman, Nalan Bastürk and Lenard Lieb. Peter, I am deeply grateful and happy for the guidance you have given me over these years. Working and discussing with you have shaped and re-shaped how I think of economics and econometrics. I also want to express my great appreciation for my co-supervisors Lenard and Nalan. Both of you helped me develop and progress even when my research projects diverged from your interests. Furthermore, I want to recognise the crucial importance Jean-Pierre Urbain had for my early academic development. I am profoundly saddened by the fact that Jean-Pierre is not among us anymore. I met Jean-Pierre in 2012 at Gothenburg University, he advised me to come to Maastricht to study the research master. He would always take the time to hear about how work and how life was going. I was then, and still am, proud to have been one of his Ph.D. students. We are many that will always remember him.

I want to thank the committee for reading and evaluating my dissertation. Thank you, Jaap Bos, Marie Lambert, Stephan Smeekes and Frank Kleibergen. Your comments and assessment will help my research further advance as I go forward. A special thanks to Frank for attending my first ever external presentation of my research at the annual Society of Financial Econometrics conference in New York back in 2017. I am also profoundly thankful to Jaap Bos and Paulo Rodriguez for all their advice and support through these years.

I want to give great thanks to my friends and colleagues both at the Department of Quantitative Economics and Department of Finance. To work with you all have been a great experience that I am deeply thankful for. Firstly, I want to thank the secretaries of both departments, Francien Schijlen, Carina Wijnands, 
Karin van den Boorn and Yolanda Paulissen. Thank you for being patient with me and my complete inability to understand university administration. I also want to extend my thanks to Andrey Kateshov, Niloufar Yousefinamesh, Alessandro Pollastri, Luca Margaritella, Hanno Reuvers, Juan Palacios, Denis de Crombrugge, Tomas Post, Stefan Straetmans, Mike Langen, Verena Jung, Vincent Kreuzen, Aida Abiad, Roland Vincze, Robert Adamek, Anne Balter, Benoit Duvocelle, Shashwat Khare, Yicong Lin, Niels Mourmans, Dewi Peerlings, Caterina Schiavoni, Li Sun, Thijs Kamma, Marina Gertzberg, Martijn Stroom, Matteo Bonetti, Marten Laudi, Colin Tissen, Nora Pankratz, Joyce Mertens, Janek Kretschmer, Inka Eberhardt, Mattijs Korevaar, Marcello Negrini, Martin Strobel among others. Thank you Niloufar, I am happy that we finally settled that I am the Jengamaster. Moritz, thank you for the time, albeit short time, we shared an office. Thank you Alessandro, we have absolutely nothing but terrific memories. Furthermore, I want to thank my friends from my time as a student, Peter Thesling, Marius Lohschelder, Philip Nadler and Jakob Hemming. Thank you Philip for the great times we had in London. Also, I want to thank Elias Tsakas and Kaj Thomsson for all their invaluable effort with regards to our job market training and preparation.

As I am looking back on my time as a Ph.D. student I feel very special nostalgia for my first year of the Ph.D. This was a remarkable time made special by my friends and colleagues Alexander, Marina, Sean, Simon, Andrey, Etiënne and Yuliya. Alex, you were a great office mate and an even better friend, I am deeply grateful for the times we have shared. Thank you Marina, for all great talks we had over coffee. Yuliya and Sean, I want to thank you both for all of our talks and discussions. Simon, I could not have been luckier than to spend my first year sharing an office with you. You showed me the way to get settled and get comfortable in the role of a Ph.D. student. Most importantly, our sign at the door reading 'Embassy of Sweden', caused much bewilderment among students. During my Ph.D. I have been fortunate enough to have people around me that can weather my sometimes less-than-sunny disposition. I cannot imagine the hardship this implies, these people are titans of humanity. My great friends Emil Brorsson and Daniel Uppström have both been of massive support to me. We have shared so much over so many years and seeing you always makes me feel home. As I moved to Maastricht, it only took days before I got to know Henrik Zaunbreacher. Thank you Henrik, you are an invaluable friend and you have been an essential support for me both during our studies and the Ph.D. I also want to 
express my deepest gratitude to Adam Jassem and Elisa Voisin, both of you have been incredibly supportive of me and always got my mind off work when I needed to.

There are several institutions apart from Maastricht University that have shaped my education and development. From my undergraduate studies in Gothenburg I want to recognize the crucial importance of Hans Bjurek and Åsa Löfgren. I once asked Hans what other topics I should add to my economic studies, he told me to complement the economics courses with either statistics or mathematics. I went with statistics and it was the best decision I ever made during my education. Åsa gave me the opportunity to be a teaching assistant for the introductory statistics courses. The great feeling I got from being a part of the educational institution made me even more certain that a Ph.D. would be a great experience. Nevertheless, I was occasionally doubting whether the Ph.D. was right for me, and whether I was right for the Ph.D. One such period of doubt was in my second year. Luckily, during that time I got the opportunity to go and work at the Bank of England. This was a fantastic experience, working with Luisa Pires and her statistics group gave me renewed energy to continue my research.

Lastly, I want to thank my family for their support and encouragement. Parents Lars Karlsson and Marianne Lönn and their respective partners Britt-Marie Johansson and Håkan Peterson, my sisters Elin Lönn and Nina Lönn, in laws Andreas Johansson and Mattias Andersson. Living so far away from all of you is often difficult for me. Every time I come to see you in Sweden I am reminded of how deeply relaxing it is to be home. Furthermore, I want to extend a very special acknowledgement to my nephews Arvid and Theo and niece Klara. Finally, even though he is long gone, I want to recognise the momentous importance of my granddad Olle. Before important presentations I center myself by remembering his summerhouse. The memory of that house with the ocean wind brushing through trees relaxes me and gives me focus.

Rasmus Lönn, September 2019 


\section{Contents}

$\begin{array}{ll}\text { Acknowledgements } & \text { i }\end{array}$

1 Introduction 1

2 Empirical asset pricing with many assets and short time series 9

2.1 Introduction . . . . . . . . . . . . . . . . . . . . . . . . . 10

2.2 Stochastic discount factor projections . . . . . . . . . . . . . . 12

2.3 Boosting Tracking Portfolios . . . . . . . . . . . . . . . . . . . . 16

2.4 Literature . . . . . . . . . . . . . . . . . . . . 20

2.5 Data . . . . . . . . . . . . . . . . . . 23

2.6 Results . . . . . . . . . . . . . . . . . . . . 27

2.6 .1 Tracking portfolios . . . . . . . . . . . . 27

2.6.2 Pricing kernel estimates . . . . . . . . . . . . . . 32

2.6.3 Mean-Variance efficient portfolio . . . . . . . . . . 34

2.6 .4 Asset pricing . . . . . . . . . . . . . . 35

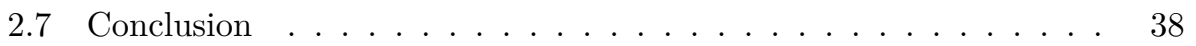

3 Optimizing large portfolios using factors and sparse hedging 41

3.1 Introduction . . . . . . . . . . . . . . . . . . . . . 42

3.2 Financial framework for portfolio optimization . . . . . . . . . 45

3.2.1 A financial framework for the inverse covariance matrix . . 46

3.2.2 Illustrating the Factor-Graphical approach by simulation . 48 
3.3 Estimating the idiosyncratic inverse . . . . . . . . . . 50

3.3 .1 The graphical lasso . . . . . . . . . . . . . . . . 51

3.3.2 Inverse estimation through Neighborhood Selection . . . . . 55

3.4 Minimum variance allocation of the $\mathrm{S} \& \mathrm{P} 500 \ldots \ldots \ldots \ldots$

3.4.1 Data and performance measures . . . . . . . . . 58

3.4.2 Factor-Graphical portfolio performance . . . . . . . . . 59

3.4.3 Decomposing the Factor-Graphical portfolio . . . . . . . . . 64

3.4.4 Investor utility and performance under re-sampling . . . . . 69

3.5 Conclusions . . . . . . . . . . . . . . . . . . . . . . . 71

3.A Simulation design . . . . . . . . . . . . . . . . 75

3.B S\&P500 financial data . . . . . . . . . . . . . . . . . 79

3.C Competing estimators: POET and Shrinkage . . . . . . . . 80

3.D Portfolio composition . . . . . . . . . . . . . . . . . 82

3.E Bootstrap distributions $\ldots \ldots \ldots \ldots$. . . . . . . . . . 83

3.F Conditional sparsity in the inverse . . . . . . . . . . . 86

\section{Pricing impacts of trade in a large cross-section of returns $\quad 91$}

4.1 Introduction . . . . . . . . . . . . . . . . . . . 92

4.2 A network of trade-price relations . . . . . . . . . . . . . . 95

4.2.1 Estimating asset neighborhoods . . . . . . . . . . . . . 96

4.2 .2 Calibrating the regularization . . . . . . . . . . . . . 99

4.2 .3 Asset and market connectedness . . . . . . . . . . . . 100

4.3 Network features and characteristics over time . . . . . . . . . 101

4.4 Is asset and market connectedness priced . . . . . . . . . . . 107

4.4.1 Asset connectedness . . . . . . . . . . . . . . . . . . 108

4.4.2 Dissecting connectedness using Size and Degree . . . . . . . 110

4.4.3 Market connectedness . . . . . . . . . . . . . . . . . . . . . 114

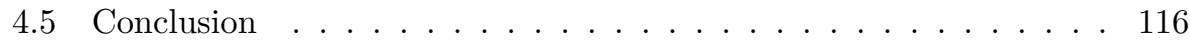

4.A Network price reversions . . . . . . . . . . . . . . . . . . 119

4.B Connectedness before and after $1995 \ldots \ldots \ldots$. . . . . 120

4.C Erdös and Renyi diameter . . . . . . . . . . . . . . . . . . . 122 
Valorization

Bibliography

Nederlandse Samenvatting

Curriculum Vitae 



\section{Chapter 1}

\section{Introduction}

"The special sphere of finance within economics is the study of allocation and deployment of economic resources, both spatially and across time, in an uncertain environment. To capture the influence and interaction of time and uncertainty effectively requires sophisticated mathematical and computational tools."

- Robert C. Merton (1944-) 
My thesis explores topics from empirical asset pricing, portfolio optimization and financial frictions. These topics are well summarized as empirical essays about the mean-variance frontier and the cross-section of returns. The common denominator, recurrent in all chapters, is how to handle the high-dimensionality and excessive model complexity that arise when the cross-section of assets is large relative to the length of the time series.

My chapters all relate to the study of the joint characteristics of many interacting assets. This may be in the context of a financial market or a portfolio. The joint behaviour of many assets implies that special consideration is given to the manner in which assets co-vary. Statistically, we are confronted with a very large number of unknown parameters to estimate, which can be difficult. This difficulty is made worse by the empirical fact that financial returns are very noisy; there is much excess volatility in equity returns (Shiller, 1981). The immediate consequence of this is that it can be very difficult to precisely discern the characteristics of asset returns, and by extension the characteristics of financial markets. Thus, from an econometric perspective the prominent issues common to the topics I cover are: (1) there is a very large cross-section of returns, (2) returns are very noisy. To tackle these issues I rely on tools from the high-dimensional statistics and machine learning literature. The tools I explore are able to accommodate the large cross-section, while also actively reducing the impact of the noisy markets. The contribution of my thesis is to engage these methods in a manner that is well-informed from the point of view of financial theory.

To contextualize the chapters of my thesis, and highlight the empirical challenges, it helps to introduce the mean-variance framework of $N$ assets financial returns. ${ }^{1}$ To analyze returns, denoted $R$, it is natural to start from the fundamental pricing conditions consistent with the utility maximization of investors. For a financial return $R_{i}$ and a stochastic discount factor (SDF), denoted $m$, the pricing conditions are,

$$
\mathrm{E}\left(m R_{i}\right)=1 .
$$

Here $m$ summarizes risk adjustments that occurs on financial markets. For a more complete treatment of these conditions see, for example, Cochrane (2005). Expanding these conditions, it is possible to form a frontier for the trade-off between risk (measured as the variance of returns) and expected return, which restricts the

\footnotetext{
${ }^{1} \mathrm{I}$ am suppressing the time dimension $T$ for cleaner notation.
} 
characteristics of the financial assets. Introducing a risk-free rate, $R_{f}$ it is possible for us to express the mean-variance frontier as,

$$
\left|\mathrm{E}\left(R_{i}\right)-R_{f}\right| \leq \frac{\sqrt{\operatorname{Var}(m)}}{\mathrm{E}(m)} \sqrt{\operatorname{Var}\left(R_{i}\right)}
$$

Thus, representing all returns by their expected returns and their variance we find that excess returns over the risk-free rate are all constrained by a wedge with the slope $\sqrt{\operatorname{Var}(m)} / \mathrm{E}(m)$. To frame the chapters of my thesis we further add economic content by introducing a model of $k$ factors $x$, to approximate the SDF. An example of what a model of some risk factor, $x$, might look like is,

$$
m=a+\delta x
$$

The identification of risk factors is a contentious topic see, e.g., Cochrane (2011). However, finding factors to proxy for risk factors still provides important insights for risk and portfolio management. ${ }^{2}$ The contribution of my thesis relates to the large- $N$ study of the mean-variance frontier and the SDF through the following questions: (1) how can we assess and compare models for $m$; (2) how can we most effectively form minimum-variance portfolios; (3) how to quantify certain market frictions to form a factor that proxy risk adjustments. For all of these purposes there follows complications from $N$ often being large relative to $T$.

Conventional tools of financial econometrics are often inappropriate to approach these topics when the number of assets is large. To tackle this issue the methodology I relied on method of statistical regularization and data-driven learning. These estimators and algorithms, while generally developed for very different applications, are especially well suited to handle large and noisy data environments. However, to specify the form of regularization, or choice of learning algorithm, I draw upon economic and financial theory to inform the methodology. In general the methods we propose are not replacements of theoretical economic and financial constraints in favor of statistical constraints. The ambition has been to propose approaches that combine economic theory and statistical learning in a mutually beneficial manner.

\footnotetext{
${ }^{2}$ The literature exploring proxies has by now introduced an additional dimensionality challenge since the number of proposed factors has grown large. Harvey, Liu, and Zhu (2016) explore those published, and in review, for top journals finding a number of factors exceeding 300 . Several papers explore what factors provide independent information about returns, among them Kozak, Nagel, and Santosh (2017); Feng, Giglio, and Xiu (2017); Gu, Kelly, and Xiu (2018).
} 


\section{Assessing macroeconomic factor models}

In chapter two of my thesis we propose an approach to assess and compare macroeconomic asset pricing models. Representing a model for the SDF as a return, we assess the fit of the model by its distance to the mean-variance frontier. Assessing this distance is however troublesome when $N$ is large. The difficulty follows from each asset providing a distinct pricing condition, see (1.1). Thus, estimating the risk prices $\delta$ from (1.3) by GMM is difficult because the optimal weighting matrix involves the inversion of an $N \times N$ matrix. Previously proposed methods that are viable in our large- $N$ framework, such as the Fama-Macbeth approach (Fama and MacBeth, 1973), are generally subject to severe errors-in-variables problems. The problem of small- $T$ is especially present for macroeconomic risk models, since macroeconomic data is currently unable to match the high-frequencies of financial markets. Factors such as consumption, industrial production, inflation are often observed on monthly basis, or even less frequent.

We propose a method that is valid and well suited to evaluate asset pricing models even when $N$ is much greater than $T$. Our method relies on the Hansen-Jagannathan distance (Hansen and Jagannathan, 1997) to decompose the parameter estimation and model evaluation into a two-stage procedure. The first stage of the assessment is a high-dimensional linear projection of $k$ factors onto $N$ predictors. From a financial perspective these projections are tracking portfolios (Lamont, 2001). These tracking portfolios are linear combination of assets that mimic the development of the news in the factors. We propose to form these portfolios using a linear L2-boosting algorithm, which is well suited to handle the large number of predictors. The second stage of our approach is a low-dimensional regression of an estimated mean-variance portfolio, onto the projections from the first stage. Belloni, Chen, Chernozhukov, and Hansen (2012) study in detail the asymptotic properties of this form of two-stage procedure, guided by their results we obtain consistent standard errors for the risk prices.

Applying our methodology we find that the classic consumption based CAPM along with other fundamental macroeconomic models only explain a small part of the variation of expected returns. While factors like industrial production growth and inflation enhance the fit of the consumption based models, these gains are modest. The most impressive gains in terms of predictive power come from allowing nonlinear transformations of consumption growth that emphasize the importance 
of negative growth. However, fundamental factors such as consumption growth and inflation are priced.

\section{Large-scale minimum variance allocation}

The next point I address in my thesis is the minimum variance allocation of assets. This is a natural continuation from Chapter 2, where I assess the degree to which a factor model spans the mean-variance frontier. In Chapter 3 focus is moved onto the minimum variance point of the frontier. In the broad context, Chapter 3 is concerned with the frontier from (1.2) whereas Chapter 2 is is mainly focused on the pricing models (1.3). Minimum variance allocation is especially important for portfolio optimization since the optimal mean-variance allocation is generally found to be empirically poor out-of-sample. Minimum-variance allocation often provides a more compelling alternative. There are by now many explanations for this including low-risk anomalies, factor exposures, compositional characteristics and behavioural explanations (Ang, Hodrick, Xing, and Zhang, 2006; Scherer, 2011; Clarke, de Silva, and Thorley, 2011; Baker, Bradley, and Wurgler, 2011).

Despite its appeal and advantages, minimum variance allocation still pose significant empirical challenges since it still relies on the inverse covariance matrix. Thus, in portfolios of many assets these allocations become burdensome to estimate since the number of unknown parameters grows quadratically in $N$. In addition, the matrix inversion may compound the measurement errors, which can be substantial due to the noisy returns.

I propose a methodology that combines financial factor models with graphical model selection to overcome the challenges of estimating the inverse. In the proposed method, the factor models provide a structure that aids the parameter estimation, the graphical model selection makes it possible to circumvent the large-scale matrix inversion. The financial intuition behind the method is that the factors accounts for common exposures, while the graphical estimation forms small hedging portfolios to compensate for deviations from the common factors. ${ }^{3}$ The method deviates from the most prominent methods of the literature, among them are the Shrinkage estimators of Ledoit and Wolf $(2003,2004,2012)$ and the factor and POET estimators of Fan, Fan, and Lv (2008) and Fan, Liao, and Mincheva (2013). The main departure from these estimators is that I target the inverse covariance matrix 'directly'. Thus, I can circumvent the need to invert the large- $N$

\footnotetext{
${ }^{3}$ The hedging portfolio interpretation is demonstrated by Stevens (1998).
} 
covariance matrix. Empirically, we find that avoiding the matrix inversion is crucial when there are many assets to consider. This feature of the method relies on a sparsity assumption on the inverse residual covariance matrix. This assumption of statistical sparsity in the inverse, instead of the covariance matrix, is a conceptual departure from prominent previous methods, among them the POET estimator.

I evaluate the method empirically using daily returns on the S\&P500 companies. The findings indicate that the method reduces volatility more than competing methods. This include prominent covariance estimators, such as the Shrinkage and POET, as well as nested approaches, including strict factor models and the sparse hedging method of Goto and $\mathrm{Xu}$ (2015). Bootstrapping the distribution of the volatility reduction we find that the improvement over the second best method, the Shrinkage estimator of Ledoit and Wolf (2003, 2004), is statistically significant. The detriment of the method I propose is an increase in the trade required to maintain the allocation.

\section{Asymmetric information and order flows}

The fourth chapter treats the topic of liquidity and asymmetric information on financial markets. Re-connecting to the mean-variance framework, we attempt to form a factor that captures these frictions such that we can improve upon current financial factor models. Thus, our interest is here to improve our understanding of, and more accurately approximate, (1.3). Our approach is to make use of regularized estimation to quantify pricing impacts from the vast cross-section of order flows. Financial theory describes how liquidity - ease of trade - decrease, and expected returns increase, in markets with large informational asymmetry (Vayanos and Wang, 2011). The basis of the connection between pricing sensitivity to trade and financial liquidity was outlined by Kyle (1985) and Caballe and Krishnan (1994). Our estimates can be understood as networks of trade-pricing relations, which as a representation is related to the approach proposed by Diebold and Yllmaz (2014). Strong connectivity in our network implies high levels of illiquidity. As a results, we treat the connectedness of each asset to this network as a factor to augment the standard factor models. We find that these impacts increase the classic Fama-French models fit to the mean-variance frontier.

From a financial perspective the network is the result of extending the empirical framework of Pastor and Stambaugh (2003) into a multivariate setting. Conceptually, this achieves two goals. First, it introduces pricing impacts as an 
addition to Pastor-Stambaughs' price reversion parameters. Secondly, it provides a representation that allows us to treat commonalities in more flexible manner compared to previous works such as Hasbrouck and Seppi (2001), Korajczyk and Sadka (2008) and Hallin, Mathias, Pirotte, and Veredas (2011).

A network connection between assets is present if changes in order flows of one asset induce price adjustments in the other. In this context, markets characterized by high levels of informational asymmetry display large price adjustments in response to trade. Thus, the development of our networks track the degree of informational asymmetry and illiquidity, both on the market and asset levels. We propose to estimate this vast network using regularization in the form of an elastic net (Zou and Hastie, 2005). The benefit of this regularization is that nests the classic lasso and ridge estimators, which provides a lot of flexibility in terms of the network characteristics we can accommodate.

As the networks develop over time, the connectedness of assets adjust to reflect the liquidity circumstances. As we track the development we find that both asset level connectedness to our network, as well as the average connectedness at a given time, are a priced factors. We also find that the characteristics of the networks augments the Fama-French factors in a statistically significant way. Furthermore, we find that issues regarding financial liquidity are particular small-stock phenomena, which is in line with what previous works on this topic has found. Additionally, we find that the predictive power of the relation between the estimated network characteristics and the cross-section of returns has increased as the magnitude of order flows has escalated.

\section{Outline}

Chapters 2 through 4 of the thesis present the detailed accounts of my the previously introduced body of work. Each chapter is introduced individually with separate conclusions given at the end. Along with each chapter follows relevant

appendices. A general conclusion of the work along with the broad implications of my findings is presented in chapter 5 , followed by a valorisation. Last follow references, a Dutch summary and a curriculum vitae. 



\section{Chapter 2}

\section{Empirical asset pricing with many assets and short time series}

We construct tracking portfolios consisting of a large number of assets for macroeconomic factors using the $L_{2}$-boosting algorithm. We use these tracking portfolios as instruments to estimate factor risk prices. The same learning algorithm also provides the weights of a mean-variance efficient portfolio. With this additional input we compute the Hansen-Jagannathan distance to compare how alternative models fit the cross-section. We apply the method to 900 portfolio return series in the Kenneth French data library. While macro factors fail to explain most crosssectional variation, we find that both consumption and inflation risk are priced. ${ }^{1}$

\footnotetext{
${ }^{1}$ This chapter is based on the paper Lönn and Schotman (2018).
} 
Our econometric techniques are all designed for large time series and small cross sections. Our data has a large cross section and short time series. A large unsolved problem in finance is the development of appropriate large- $N$ small- $T$ tools for evaluating asset pricing models.

(Cochrane, 2005, p 226)

\section{$2.1 \quad$ Introduction}

A recurring problem in empirical asset pricing is the need to invert a largedimensional covariance (or second moment) matrix. For example, in a standard time series based asset pricing test a small number of factors is proposed to explain the differences in expected returns across a large number of assets. The null hypothesis is that Jensen's alpha should be zero for all $N$ test assets. But doing the test when $N$ is large relative to the length of the series $T$, or as in our application even bigger than $T$, is problematic. We will formulate and interpret standard asset pricing tests in an instrumental variables regression framework that allows application of statistical learning techniques to deal with the big- $N$ problem. In this framework explicit inversion of an $N$-dimensional moment matrix to compute a standard least squares regression will be infeasible. Our approach builds on an insight from Hansen and Jagannathan (1997) defining the distance between a valid stochastic discount factor (SDF) $m^{*}$ - that correctly prices all assets - and an asset pricing model $\mathrm{m}$. In the model the discount factor is a function of a few non-traded economic factors, whereas the valid $m^{*}$ is a function of all $N$ returns. We decompose their $H J$ distance measure such that we can estimate the factor risk prices $\delta$ through a time series regression of the constant ' 1 ' on the factors $x$

$$
1=\delta^{\prime} x+u
$$

using the $N$ excess returns as instruments. Since the number of instruments is large, the econometric problem is how to select a linear combination of the instruments. As proposed in Belloni, Chen, Chernozhukov, and Hansen (2012) the optimal instruments are selected by machine learning. The resulting instrument can be interpreted as tracking portfolios. For the $H J$ distance itself we need to replace the constant on the left hand side of (2.1) by a further tracking portfolio for $m^{*}$, which we obtain from the same machine learning algorithm. The mean residual squared error is then the $H J$-distance. 
Given the instruments, estimation and evaluation of the model only involves standard time series regressions. The big- $N$ operations deal with the selection of assets that are most informative about the economic factors. This separates our approach from the Fama-Macbeth methodology, which uses cross-sectional regressions in which all assets are weighted equally.

Our main empirical contribution is the use of statistical learning, both to construct tracking portfolios for standard macroeconomic variables, such as consumption, inflation, interest rates and unemployment, as well as to test if these are priced. Tracking portfolios for macroeconomic portfolios have been used often in asset pricing studies, but always using just a few (between 5 and 15) assets in the factor mimicking portfolio. In contrast we include about 900 candidate assets: all US equity portfolios available on Kenneth French's online data library. This is a big- $N$ regression problem. A large literature has developed for constructing sparse regression fits with many potential explanatory variables and a relatively small number of observations. Since a portfolio return is a linear combination of asset returns, we use an additive learning algorithm. The $L_{2}$-boosting algorithm (Friedman, 2001; Bühlmann, 2006) is promising in this respect, since it allows for many small coefficients when $N$ grows large. In contrast, $L_{1}$-type techniques, such as LASSO (Tibshirani, 1996), generate a sparse portfolio with a small number of large coefficients and many zero weights. With many candidate assets overfitting becomes a major concern. To avoid overfitting, we tune the boosting algorithm both by an information criterion that penalizes model complexity, as well as randomized cross-validation, which estimates on a random subset of the data and evaluates on the remaining data.

The tracking portfolio for the valid stochastic discount factor is an unrestricted mean-variance (MV) efficient portfolio. Technically, Jobson and Korkie (1983) already demonstrated that this problem can be represented as a regression of a vector of ones on the collection of $N$ excess returns. For this regression problem we employ the same $L_{2}$-boosting algorithm.

In the remainder of the chapter we start by reviewing estimation of a stochastic discount factor model and the Hansen-Jagannathan distance to assess its crosssectional fit. We then provide the details of the machine learning algorithm that we use to construct tracking portfolios. After explaining what we do, we discuss how our method relates to the literature, and especially to some recent studies that apply machine learning tools to asset pricing problems. The second part of the chapter contains the empirical application using a small number of macroeconomic 
factors and a large number of return portfolios. In the conclusions we discuss issues for further research.

\subsection{Stochastic discount factor projections}

Let $y$ be a vector of excess returns on $N$ different assets or portfolios of assets. The stochastic discount factor model states that the excess returns satisfy the $N$ moment conditions

$$
\mathrm{E}[m y]=0,
$$

where $m$ is a stochastic discount factor (SDF). The model is given economic meaning by specifying a functional form for the discount factor. We will consider linear models for the discount factor of the form

$$
m=1-\delta^{\prime} x
$$

for a set of $M$ macroeconomic factors $x$ and parameters $\delta$. Since we are modeling excess returns, the intercept of "1" is an arbitrary normalization parameter. The two interesting questions are how well the discount factor model can explain the cross-section of expected returns, and which economic factors are priced. The two questions are related, as $\delta$ is estimated to maximize the model fit in a chosen metric.

Ideally the factors explain the entire cross-section of expected returns, in which case all the moment conditions (2.2) hold exactly. In practice, anomalies exist either due to mispricing or due to omitted factors. If the moment conditions are only approximate, the deviations $\mathrm{E}[m y]$ in (2.2) are pricing errors. Hansen and Jagannathan (1997) propose a distance measure to evaluate the fit of the stochastic discount factor model. Their distance measure is defined as

$$
H J=\min _{m^{*} \in \mathcal{M}} \mathrm{E}\left[\left(m-m^{*}\right)^{2}\right],
$$

where $\mathcal{M}$ is the set of all valid discount factors that satisfy the pricing condition $\mathrm{E}\left[m^{*} y\right]=0$ for the set of $N$ test assets. The criterion finds a valid discount factor $m^{*}$ that is closest to the model $m$ in (2.3). The solution is

$$
H J=\mathrm{E}[m y]^{\prime} \mathrm{E}\left[y y^{\prime}\right]^{-1} \mathrm{E}[m y] .
$$


This is a quadratic form in the pricing errors $\mathrm{E}[m y]$ with weighting matrix $\mathrm{E}\left[y y^{\prime}\right]^{-1}$. Hansen and Jagannathan (1997) discuss the difference between the distance measure $H J$ and a GMM weighting matrix. Most importantly the $H J$ weighting matrix, $\mathrm{E}\left[y y^{\prime}\right]^{-1}$, assures that results are invariant to repackaging of the assets. Forming portfolios of the original assets does not change the $H J$ distance. In addition the Hansen-Jagannathan weighting matrix is independent of the model for $m$, which facilitates model comparisons.

When the parameters $\delta$ are unknown the distance is further minimized with respect to $\delta$, minimizing $(2.5)$ we find,

$$
\delta=\left(\mathrm{E}\left[x y^{\prime}\right] \mathrm{E}\left[y y^{\prime}\right]^{-1} \mathrm{E}\left[y x^{\prime}\right]\right)^{-1} \mathrm{E}\left[x y^{\prime}\right] \mathrm{E}\left[y y^{\prime}\right]^{-1} \mathrm{E}[y] .
$$

In applications the population moments in (2.5) and (2.6) are replaced by sample moments assuming that we have a sample of $T$ observations for both $x$ and $y$. When $N$ is large relative to $T$, the weighting matrix contains $O\left(N^{2}\right)$ elements to be estimated. Finding any quantity depending on $\mathrm{E}\left[y y^{\prime}\right]^{-1}$ involves a huge matrix inversion that can be very sensitive to estimation error. In the really big- $N$ case, when $N>T$, the sample second moment matrix of excess returns will even be singular.

Our approach circumvents the need to explicitly estimate the large $(N \times N)$ weighting matrix $\mathrm{E}\left[y y^{\prime}\right]^{-1}$. For this we interpret the estimator (2.6) as the instrumental variables (IV) estimator of $\delta$ in the regression

$$
1=\delta^{\prime} x+u
$$

using $y$ as instruments. To implement the IV estimator we perform a first stage regression to construct the tracking portfolios

$$
\hat{x}=\operatorname{Proj}(x \mid y)=\mathrm{E}\left[x y^{\prime}\right] \mathrm{E}\left[y y^{\prime}\right]^{-1} y
$$

The second stage IV estimator then becomes

$$
\hat{\delta}=\mathrm{E}\left[\hat{x} x^{\prime}\right]^{-1} \mathrm{E}[\hat{x} 1],
$$

which is identical to the original expression (2.6). For our purpose the main difference between (2.6) and (2.9) is the dimension of the matrix inversion. In (2.6) we need the large dimensional $\mathrm{E}\left[y y^{\prime}\right]^{-1}$, whereas in (2.9) we only have the 
low dimensional $\mathrm{E}\left[\hat{x} x^{\prime}\right]^{-1}$. The big- $N$ challenge in (2.9) is in the tracking portfolio $\hat{x}$. Our approach is to construct this portfolio with tools from machine learning designed to fit regressions with a large number of explanatory variables.

The IV formulation puts our model directly within the framework of Belloni, Chen, Chernozhukov and Hansen (2012). They consider the problem of instrument selection in a regression model with a fixed small number of endogenous regressors $x$, for which we have many instruments $y$. All $y_{j}$ are potential instruments, but including too many is not efficient and will lead to biases. In a first stage a penalized regression provides $\hat{x}$ as a linear combination of the instruments $y$. Using $\hat{x}$ as a second stage instrument, the IV estimator for $\delta$ has covariance matrix

$$
\operatorname{Var}(\sqrt{T} \hat{\delta})=\omega^{2} \mathrm{E}\left[\hat{x} x^{\prime}\right]^{-1} \mathrm{E}\left[\hat{x} \hat{x}^{\prime}\right] \mathrm{E}\left[x \hat{x}^{\prime}\right]^{-1}
$$

with $\omega^{2}=\mathrm{E}\left[u^{2}\right]$ is the variance of the residuals in the stochastic discount factor model (2.7). When instruments are chosen optimally, this reduces to

$$
\operatorname{Var}(\sqrt{T} \hat{\delta})=\omega^{2} \mathrm{E}\left[\hat{x} \hat{x}^{\prime}\right]^{-1}
$$

Under sparsity conditions for the projections, Belloni et al (2012) prove that various machine learning methods lead to asymptotically optimal instruments. One of the methods that fits within their analysis is the $L_{2}$-boosting algorithm we use in our empirical work and which we discuss in section 2.3 below.

The economic tracking portfolios are all we need to estimate risk prices $\delta$. Evaluating the model fit, however, requires one additional projection. Using the $H J$ distance we let $\hat{m}=\mathrm{E}[m y]^{\prime} \mathrm{E}\left[y y^{\prime}\right]^{-1} y$ be the projection of $m$ onto the excess returns $y$. The $H J$ distance can be rewritten as

$$
\begin{aligned}
H J & =\mathrm{E}\left[\left(\mathrm{E}[m y]^{\prime} \mathrm{E}\left[y y^{\prime}\right]^{-1}\right) y y^{\prime}\left(\mathrm{E}\left[y y^{\prime}\right]^{-1} \mathrm{E}[m y]\right)\right] \\
& =\mathrm{E}\left[\hat{m}^{2}\right]
\end{aligned}
$$

This expresses the distance as the squared projection of the discount factor on the excess returns. From the definition of the linear discount factor model, $m=1-\delta^{\prime} x$, we can split the projection in two parts,

$$
\hat{m}=\hat{1}-\delta^{\prime} \hat{x}
$$


The new element in (2.13) is the projection $\hat{1}$, which is defined as the fitted values from regressing the vector of ones on the space of excess returns. The constant tracking portfolio $\hat{1}$ is nothing but the excess return of a mean-variance efficient portfolio. Once we have the projections, the $H J$ distance can now be estimated as the second moment $\mathrm{E}\left[\hat{m}^{2}\right]$.

Another way to look at the $H J$ distance is as the expected squared residual $\mathrm{E}\left[v^{2}\right]$ from the time series regression

$$
\hat{1}=\delta^{\prime} \hat{x}+v
$$

The left-hand of this regression is the (excess) return on a mean-variance efficient portfolio, while the right-hand side variables represent a collection of portfolio returns. With return data, evaluation of the stochastic discount factor model only involves a time series regression of $\hat{1}$ on a set of macroeconomic tracking portfolios $\hat{x}$. The regression assesses how well a mean-variance efficient portfolio can be explained by the excess returns of macroeconomic tracking portfolios. If the tracking portfolios span the efficient frontier, the residual will be zero. The $H J$ distance is measured by the Mean-Squared-Error (MSE) of the regression. Adding an intercept to (2.14) we can further decompose the MSE into a bias and variance component as in standard spanning tests (Huberman and Kandel, 1987).

With the complete set of $N$ instruments, the IV estimator (2.9) using the individual tracking portfolios as instruments, is numerically identical to the least squares estimator for $\delta$ in (2.14). In terms of data matrices, if $\boldsymbol{Y}$ is the $(T \times N)$ matrix of sample excess returns, and $\boldsymbol{X}$ the $(T \times M)$ matrix of macroeconomic news data, then the IV estimator

$$
\hat{\delta}=\left(\hat{\boldsymbol{X}}^{\prime} \boldsymbol{X}\right)^{-1} \hat{\boldsymbol{X}}^{\prime} 1
$$

equals the least squares estimator

$$
\tilde{\delta}=\left(\hat{\boldsymbol{X}}^{\prime} \hat{\boldsymbol{X}}\right)^{-1} \hat{\boldsymbol{X}}^{\prime} \hat{1}
$$

when we use the unrestricted $(T \times T)$ projection matrix $\boldsymbol{H}=\boldsymbol{Y}\left(\boldsymbol{Y}^{\prime} \boldsymbol{Y}\right)^{-1} \boldsymbol{Y}^{\prime}$ to generate the projections $\hat{\boldsymbol{X}}=\boldsymbol{H} \boldsymbol{X}$ and $\hat{1}=\boldsymbol{H}$. The identity holds because the projection is idempotent, i.e. $\boldsymbol{H}^{2}=\boldsymbol{H}$, implying $\hat{\boldsymbol{X}}^{\prime} \boldsymbol{X}=\hat{\boldsymbol{X}}^{\prime} \hat{\boldsymbol{X}}$. However, when different macro variables use different optimal instruments we have $\hat{\boldsymbol{x}}_{j}=\boldsymbol{H}_{j} \boldsymbol{x}_{j}$ with individual projection matrices $\boldsymbol{H}_{j}\left(\boldsymbol{x}_{j}\right.$ is the $j^{\text {th }}$ column of $\left.\boldsymbol{X}\right)$. In that case 
the two estimators are no longer identical. To estimate the prices of risk we will use the IV estimator (2.15). For the more informal assessment whether the tracking portfolios span the efficient frontier we use the least squares estimator (2.16).

\subsection{Boosting Tracking Portfolios}

We construct tracking portfolios to deal with the large number of asset returns. Tracking portfolios have been explored in detail by Lamont (2001). His specification starts from the time series regression

$$
X_{t}=\beta^{\prime} y_{t}+\phi^{\prime} Q_{t-1}+\epsilon_{t}
$$

where $X_{t}$ is the observation at time $t$ for some macroeconomic state variable, $y_{t}$ is the $N$-vector of excess returns at time $t$ and $Q_{t-1}$ a $q$-vector of predetermined variables such as a constant term and lagged values of $X$. The tracking portfolio is defined as the linear combination $\hat{x}_{t}=\beta^{\prime} y_{t}$. As in Lamont (2001) we work with excess returns to avoid imposing the portfolio restriction that elements of $\beta$ sum to one. As emphasized by Lamont (2001) a large number of variables in $y_{t}$ may lead to overfitting and spurious results.

When $N$ is large relative to $T$, sparsity conditions are needed to make this a feasible regression. This can be done in many ways, for example by Bayesian shrinkage priors or by imposing zero restrictions as in LASSO. The boosting algorithm that we employ works under the assumption that $\sum_{j=1}^{N}\left|\beta_{j}\right|$ is bounded as $N$ increases. Adding more returns should lead to a more diversified tracking portfolio with smaller individual coefficients $\left|\beta_{j}\right|$. In contrast to the standard tracking portfolio where the assets are specified a priori, we are constructing a portfolio sequentially by adding assets that complement each other in terms of predictive properties.

Boosting algorithms were developed as a technique for producing a projection by aggregating weak predictors. The statistical foundation of the algorithm is derived from the functional gradient descent representation established by Breiman (1998). The version we use here closely follows Bühlmann (2006). It is applicable to continuous response variables and known as $L_{2}$-boosting with component-wise linear least squares.

Our empirical implementation deviates slightly from the specification in (2.17). Instead of the multiple regression with both $y_{t}$ and $Q_{t-1}$ we first regress $X_{t}$ on the 
fixed regressors $Q_{t-1}$ before starting the boosting algorithm. Moreover, since it is often unclear at what date the macro variables are known, we use the quarterly change in the data, which we center at the middle month of the quarter for aligning with the return data. The residuals $x_{t}=X_{t}-\operatorname{Proj}\left(X_{t} \mid Q_{t-1}\right)$ have mean zero, and therefore preserve the scaling of the discount factor specification. We define these residuals as the macro news factors in our model.

With this construction we obtain the following regression model relating macro news to excess returns,

$$
\boldsymbol{x}=\sum_{j=1}^{N} \beta_{j} \boldsymbol{y}_{j}+\boldsymbol{e}
$$

where $\boldsymbol{x}$ is the $T$-vector of dependent variables and $\boldsymbol{y}_{j}$ are $T$-vectors of observations on the explanatory variables. This model is the basis for the model selection procedure, summarized in Algorithm 1.

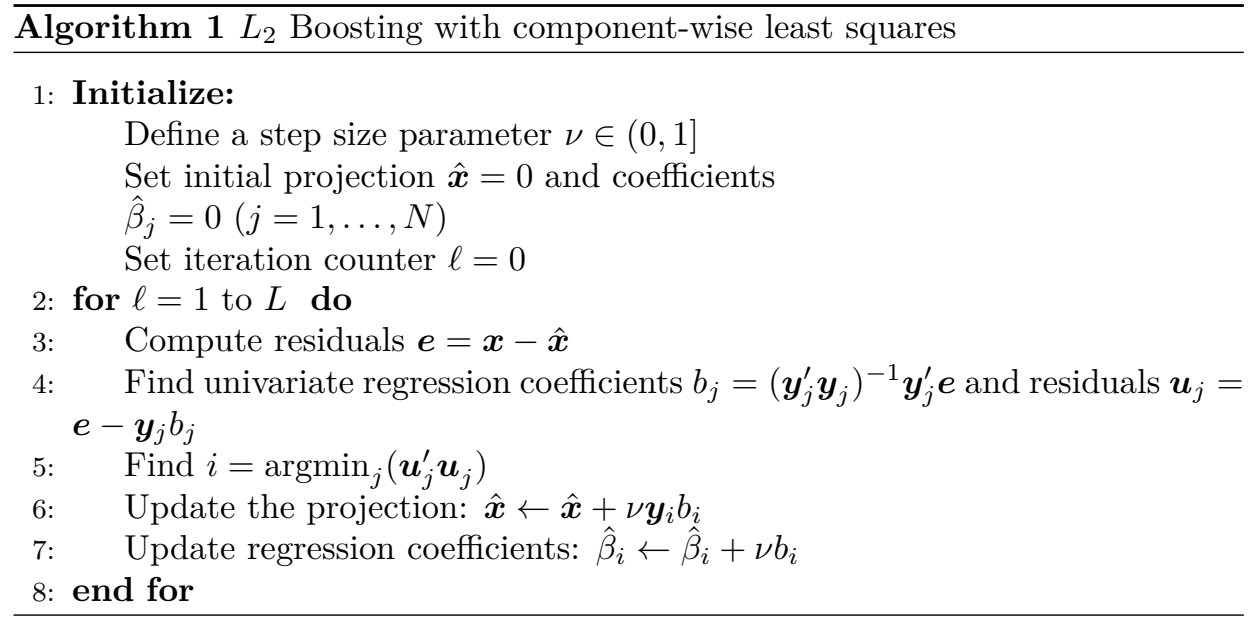

At each iteration the algorithm searches for the univariate predictor that improves the fit the most. Algorithm 1 conducts this search based on univariate regressions of the current residuals on the explanatory variables. All predictors are assessed individually and the predictor that provides the best fits of the current residuals is chosen. The projection is updated by the newly selected regressors with a shrinkage parameter $\nu$.

Boosting has two important tuning parameters: the step size $\nu$ and the number of iterations $L$. The literature concludes that the exact value of the tuning pa- 
rameter does not have much of an effect on the results, as long as it is sufficiently small. The value $\nu=0.1$ is often recommended. The step size should be large enough for the algorithm to make progress, yet small enough to enable shrinkage and deal with multicollinearity. The number of iterations $L$ is more critical, since eventually the solution will converge to the least squares solution and therefore be prone to severe overfitting.

To avoid overfitting the actual number of iterations should be set based on an information criterion or through cross-validation. In our empirical work we consider both options. For the information based stopping rule we use the Bayesian Information Criterion $(B I C)$ defined as

$$
B I C=\ln \left(\boldsymbol{e}^{\prime} \boldsymbol{e}\right)+k \frac{\ln T}{T},
$$

where $k$ is the model complexity. For the definition of the model complexity parameter $k$ we follow Bühlmann (2006). The fitted values after $L$ iterations can be expressed as

$$
\hat{\boldsymbol{x}}=\boldsymbol{H}^{(L)} \boldsymbol{x}
$$

with $\boldsymbol{H}^{(L)}$ the $(T \times T)$ pseudo projection (hat) matrix

$$
\begin{aligned}
\boldsymbol{H}^{(L)} & =\boldsymbol{H}^{(L-1)}+\nu \boldsymbol{P}_{i_{L}}\left(\boldsymbol{I}-\boldsymbol{H}^{(L-1)}\right) \\
& =\boldsymbol{I}-\prod_{\ell=1}^{L}\left(\boldsymbol{I}-\nu \boldsymbol{P}_{i_{\ell}}\right)
\end{aligned}
$$

The hat matrix aggregates the iterations using the univariate projection matrices $\boldsymbol{P}_{i}=\boldsymbol{y}_{i}\left(\boldsymbol{y}_{i}^{\prime} \boldsymbol{y}_{i}\right)^{-1} \boldsymbol{y}_{i}^{\prime}$ starting from $\boldsymbol{H}^{(0)}=0$ and using the selected regressors $i_{\ell}$ in iteration $\ell$. The matrix $\boldsymbol{H}^{(L)}$ is a pseudo projection matrix since it is not idempotent. The complexity parameter after $L$ iterations is then defined as

$$
k=\operatorname{tr}\left(\boldsymbol{H}^{(L)}\right)
$$

In standard regression models this definition of $k$ would be equal to the number of explanatory variables. Due to the shrinkage parameter $\nu$ in the boosting algorithm $k$ is less than the number of included variables, and also much smaller than the number of iterations $L$. The number of iterations $L^{*}$ that minimizes the $B I C$ criterion defines the final projection matrix $\boldsymbol{H}^{\left(L^{*}\right)}$ 
The alternative stopping rule is based on cross validation. With $J$-fold crossvalidation we split the sample in $J$ equal-sized subsamples. For each fold $j$ we run the boosting algorithm for $L$ steps on the complement of all data not in the $j^{\text {th }}$ subsample. We then construct predicted values for the $j^{\text {th }}$ subsample using the estimated parameters $\hat{\beta}^{(L, j)}$. Doing this for all $j$ gives a sample of $T$ test residuals. The value of $L$ that minimizes the residual sum of squares of the test residuals determines the optimal number of iterations. With this $L^{*}$ we then run the boosting algorithm one more time on the full sample to construct the tracking portfolio

$$
\hat{\boldsymbol{x}}=\boldsymbol{Y} \hat{\beta}^{\left(L^{*}\right)} .
$$

In practice we use 5 -fold cross validation with random subsamples. We randomly draw (without replacement) the time series observation numbers to be part of subsample $j$. To minimize the sampling variation induced by the random subsample assignment we repeat this 10 times and compute the cumulative sum of squares over all 10 cross-validation samples. We construct a separate tracking portfolio for each of the $M$ macroeconomic variables in the SDF model.

The boosting algorithm is used in the same way to construct the mean-variance optimized portfolio $\hat{1}$. Compared to the macroeconomic tracking portfolios the only difference is the initial condition. The vector of ones is not prefiltered in any way before being used as the dependent variable.

Bühlmann (2006) shows that the $L_{2}$-boosting algorithm is consistent for the conditional expectation of the response if the regression coefficients in (2.18) satisfy a sparsity condition. More precisely, with growing $T$ and $N$, maintaining that $\ln N / T \rightarrow 0$, the sparsity condition $\sum_{j=1}^{N}\left|\beta_{j}\right|=o(\sqrt{T / \log (N)})$ as $T \rightarrow \infty$ leads to the consistent projection

$$
\|\boldsymbol{Y}(\hat{\beta}-\beta)\| / T=o_{p}(1),
$$

subject to further regularity conditions on the existence of moments for $x$ and $y$ (Bühlmann and Van de Geer, 2011). The theoretical foundation of the algorithm is attractive for our purposes because of the sparsity condition. With more data the model may become more complex and the number of assets in the tracking portfolio may grow very quickly, but the weight of each individual asset should shrink. 
Boosting for instrument selection was suggested by Bai and Ng (2009). Belloni, Chen, Chernozhukov and Hansen (2012) explicitly propose it as an alternative to LASSO for these purposes. While there are clear differences between the LASSO and $L_{2}$-boosting the methods do share a number of similarities. Obvious similarities are found in how both methods perform simultaneous model selection and shrinkage. The crucial difference between them is in the form of regularization that controls the model complexity. The LASSO penalizes according to the $L_{1}$ norm, while boosting is an $L_{2}$ procedure. This difference has consequences for the type of sparsity that they support and produce. The LASSO assumes that just a few instruments have non-zero coefficients, whereas boosting allows for many small coefficients with a bound on their sum of absolute values. Generally, the latter seems more suitable for a portfolio interpretation. With many assets, diversification would usually imply that all assets have small weights. The distinction is not completely clear, however. One could consider the case that some of the assets being considered are themselves well diversified portfolios like the market portfolio or the Fama-French factors. Including such well-diversified assets blurs the distinction between 'many small' and 'few large' coefficients.

Although being an $L_{2}$ learning algorithm, boosting differs from ridge regression. In ridge regression all coefficients are shrunk. With boosting, the final result will still have many exact zeros. Hastie, Tibshirani and Friedman (2009) evaluate the boosting algorithm as one of best 'off-the-shelf' choices for data mining.

\subsection{Literature}

Using tracking portfolio for macroeconomic factors has a long history in empirical asset pricing. Examples are Lamont (2001), Vassalou (2003), Balduzzi and Robotti (2008), Aretz, Bartram, and Pope (2010). Most distinguishing in our approach is the use of machine learning tools in constructing tracking portfolios from a large set of candidate assets.

When dealing with a large cross-section, the most common empirical procedure follows the Fama and MacBeth (1973) two-stage methodology. The first stage consists of estimating covariances of returns with the factors; the second stage is a cross-sectional regression of average returns on these estimated covariances. Expected returns are $\mathrm{E}[y]$, while covariances are in the moment matrix $\mathrm{E}[y x]$ (given that macro news has zero mean). In population the FamaMacBeth estimator then 
results in

$$
\tilde{\delta}=\left(\mathrm{E}\left[x y^{\prime}\right] \mathrm{E}\left[y x^{\prime}\right]\right)^{-1} \mathrm{E}\left[x y^{\prime}\right] \mathrm{E}[y]
$$

Compared to our estimator (2.6), resulting from minimizing the $H J$ distance, the FamaMacBeth estimator has replaced $\mathrm{E}\left[y y^{\prime}\right]^{-1}$ by the identity matrix. Instead of setting it to an identity our tracking portfolios are designed to put more emphasis on those assets that have a stronger correlation with the macro factors. The $L_{2^{-}}$ boosting algorithm takes all the covariances between returns and factors as input, but selects and weights them in forming tracking portfolios. For macro factor $j$ with news time series $\boldsymbol{x}_{j}$, we don't use the unrestricted regression estimate $\hat{\beta}_{j}=\left(\boldsymbol{Y}^{\prime} \boldsymbol{Y}\right)^{-1} \boldsymbol{Y}^{\prime} \boldsymbol{x}_{j}$, which involves the infeasible $\left(\boldsymbol{Y}^{\prime} \boldsymbol{Y}\right)^{-1}$, but instead find the shrinkage portfolio weights $\hat{\beta}_{j}$ from (2.23). We thus obtain a number of implicit estimates for $\mathrm{E}\left[y y^{\prime}\right]^{-1}$, one for each factor.

The tracking portfolio not only leads to a different estimator for risk prices, but also alleviates some of the problems with assessing the fit of cross-sectional OLS. The cross-sectional $R^{2}$ is not a very good measure of fit for asset pricing models (Lewellen, Nagel, and Shanken, 2010). A GLS based $R^{2}$ or the $H J$-distance are better measures, but both require $\mathrm{E}\left[y y^{\prime}\right]^{-1}$. Lewellen et al. (2010) also recommend to use a large cross-section of test assets, which is indeed central to our approach.

Kleibergen (2009) and Kleibergen and Zhan (2018) express doubts about the correlation between macroeconomic factors and financial returns. Following earlier work by Kan and Zhang (1999) they suspect that macroeconomic factors may only have weak correlation with returns. If used in a cross-sectional regression these weak instruments can provide misleading inference. Our tracking portfolio aggregates the returns that are most highly correlated with the factors, choosing from a large set of potential assets. Obviously, if the tracking portfolios are not sufficiently correlated with the macro news variables, the second stage instrumental variable regression can only create noise. An important diagnostic of the tracking portfolios is their correlation with the factor they are supposed to mimic. As Bryzgalova (2016) we would have more confidence in factors that correlate with a broad set of instruments, which in our case would be a well-diversified tracking portfolio.

Our big- $N$ approach differs from a number of other recent studies that employ machine learning techniques to estimate the risk prices in a stochastic discount factor model. Feng, Giglio, and Xiu (2019) specify a linear SDF model for $m$, 
and consider the problem that $M$, the dimension of $x$, can be very large. Doing so they address the problem of finding which factors are priced within a large set of potential factors, coined the factor zoo by Cochrane (2011). Their factors are returns derived from portfolio sorts, which implies that they don't need to construct tracking portfolios. We assume that factors are given, and instead consider the evaluation of a pricing model using the selected factors. In our case the dimension $M$ of potential macroeconomic factors is small, and only the number of assets $N$ is large. That puts our emphasis on determining which portfolios are most informative for estimating risk prices for a given set of factors.

Our focus is on macroeconomic risk factors as opposed to characteristic sorted portfolios. Searching for priced macro factors using the cross-section of returns goes back to Chen, Roll, and Ross (1986). Since our current methodology is for a fixed number of factors, we only include some of the basic macro variables, without an exhaustive model selection on the large number of potential macro variables reviewed in Cochrane (2017).

One of our tracking portfolios is the projection of excess returns on a vector of ones, which identifies the weights of a mean-variance efficient portfolio. This part of our approach is related to Kozak, Nagel and Santosh (2017). We estimate the weights from the time series regression

$$
1=w^{\prime} y_{t}+u_{t}
$$

Kozak, Nagel and Santosh (2017) multiply both sides of (2.26) by $y_{t}^{\prime}$ and take the time series average. The left hand side then becomes the $N$-vector of mean excess returns, while the right hand side gives the $(N \times N)$ matrix of second moments. This is equivalent to a cross-sectional regression of means on covariances with $N$ observations and $N$ explanatory variables. Kozak, Nagel and Santosh (2017) apply machine learning techniques to this derived regression model assuming that the return covariance matrix is free of measurement error and can be used as fixed regressors.

Constructing a mean-variance efficient portfolio is notoriously difficult, especially when the cross-sectional dimension $N$ is large relative to the time series sample size $T$. Dangers of overfitting when $N$ is large and cross-correlations are substantial have been pointed out many times. Classic references are Jobson and Korkie (1982) and more recently DeMiguel, Garlappi and Uppal (2009). Many shrinkage and dimension reduction techniques have been suggested to obtain port- 
folios with reasonable out-of-sample performance. Our $L_{2}$-boosting technique is, as far as we know, new in this respect. A direct horse-race between $L_{2}$-boosting and state-of-the-art portfolio optimization techniques is outside the scope of the present chapter.

We take our test assets $y$ as a given collection of characteristic sorted portfolios from the data library of Kenneth French. These are not individual stocks, but managed portfolios. Other studies, such as Kozak, Nagel and Santosh (2017) construct portfolios from individual stocks. Kelly, Pruitt, and Su (2018a) simultaneously construct factors and portfolios from individual returns. Freyberger, Neuhierl and Weber (2017) use machine learning techniques to find functions of characteristics that explain cross-sectional differences in expected returns, which could lead to an even larger and more informative set of test assets.

\subsection{Data}

The financial data used for the construction of the tracking portfolios are all collected from the sorted equity portfolios maintained on the website of Kenneth French. We include the domestic US equity portfolios. Table 2.1 provides the list of the data that we consider for the tracking portfolios. In total the candidate set of instruments contains $N=901$ excess returns, all observed on a monthly frequency from January 1965 to February 2016, providing $T=638$ time series observations. Hence the final set of asset candidates is maintaining a cross-sectional dimension well in excess of the time dimension of the data.

The sorted returns contain several redundancies. For example, quintile portfolios are approximately the average of two decile portfolios. Similarly, double sorted portfolios on size and another characteristic can be aggregated to single sorts on the characteristics. For the boosting algorithm these redundancies are not a problem when constructing macroeconomic tracking portfolios. However, they do lead to some near arbitrage relations when we construct the unit tracking portfolio. If the dependencies would be exact, this would not cause any problems for the boosting algorithm. But with near exact linear dependence, the model selection for the constant mimicking portfolio will identify faux arbitrage portfolios by leveraging on the very small differences in the linear dependencies. For the Mean-Variance efficient portfolio we therefore only include the highest order quantiles in the candidate set, i.e. we take deciles instead of quintiles. We further drop the univariate sorts when a bivariate sort with size is also available. With the 
Table 2.1: Portfolios included from the Kenneth French data library

\begin{tabular}{|c|c|c|}
\hline$N$ & Portfolios formed on & File name \\
\hline 18 & Size & Portfolios_Formed_on_ME \\
\hline 19 & Book-to-Market & Portfolios_Formed_on_BE-ME \\
\hline 18 & Investment & Portfolios_Formed_on_INV \\
\hline 18 & Operating Profitability & Portfolios_Formed_on_OP \\
\hline 10 & Momentum & 10_Portfolios_Prior_12_2 \\
\hline 10 & Short-Term Reversal & 10_Portfolios_Prior_ $1 \_\overline{0}$ \\
\hline 10 & Long-Term Reversal & 10_Portfolios_Prior_60_13 \\
\hline 19 & Earnings/Price & Portfolios_Formed_on_E-P \\
\hline 19 & Cashflow/Price & Portfolios_Formed_on_CF-P \\
\hline 19 & Dividend Yield & Portfolios_Formed_on_D-P \\
\hline 100 & Size and BtM & 100_Portfolios_10x10 \\
\hline 99 & Size and Op. Prof. & 100_Portfolios_ME_OP_10x10 \\
\hline 100 & Size and Inv. & 100_Portfolios_ME_INV_10x10 \\
\hline 25 & BtM and Op. Prof. & 25 Portfolios_BEME_OP_ $\_5 \times 5$ \\
\hline 25 & BtM and Inv. & 25_Portfolios_BEME_INV_5x5 \\
\hline 25 & Op. Prof. and Inv. & $25 \_$Portfolios_OP_INV_ $5 \times \bar{x}$ \\
\hline 25 & Size and Momentum & 25_Portfolios_ME_Prior_12_2 \\
\hline 25 & Size and STR & 25_Portfolios_ME_Prior_1_0 \\
\hline 25 & Size and LTR & 25_Portfolios_ME_Prior_60_13 \\
\hline 25 & Size and Accruals & 25_Portfolios_ME_AC_5 55 \\
\hline 25 & Size and Market Beta & 25_Portfolios_ME_BETA_5x5 \\
\hline 25 & Size and Variance & 25_Portfolios_ME_VAR_5x5 \\
\hline 25 & Size and Residual Variance & 25_Portfolios_ME_RESVAR_5x5 \\
\hline 35 & Size and Net Share Issues & 25_Portfolios_ME_NI_5x5 \\
\hline 32 & Size, BtM, and Op. Prof. & 32_Portfolios_ME_BEME_OP_2x $4 \times 4$ \\
\hline 32 & Size, BtM, and Inv. & 32_Portfolios_ME_BEME_INV_2x $4 \times 4$ \\
\hline 32 & Size, Op. Prof., and Inv. & 32_Portfolios_ME_OP_INV_2x $\overline{4} \times 4$ \\
\hline 48 & Industry & 49_Industry_Portfolios \\
\hline 5 & Fama-French Factors & F-F_Eesearch_Data_5_Factors_2x3 \\
\hline 1 & Long-term Reversal & F-F_LT_Reversal_Factor \\
\hline 1 & Momentum & F-F_Momentum_Factor \\
\hline
\end{tabular}

Data: http://mba.tuck.dartmouth.edu/pages/faculty/ken.french/data_library.html. The table lists the file names as they are recorded on the website dataverse. From all files we use the value weighted portfolios. Abbreviations: Op. Prof. = Operating Profitability, Inv. = Investments, BtM = Book-to-Market, STR = Short-Term-Reversals, LTR $=$ Long-Term-Reversals. Some of the sets contain fewer variables than in the original data library due to missing values. A time series of returns is excluded from our database if more than one year of returns is missing in our sample Jan 1965- Feb 2018. With fewer missing values we replace the missing data by the riskfree rate. 
Table 2.2: Portfolio summary statistics

\begin{tabular}{lrcrcrc}
\hline \hline & Mean & St.dev & Min & Median & Max & Market \\
\hline Average & 0.69 & 0.22 & -0.20 & 0.69 & 1.43 & 0.52 \\
Std dev & 4.89 & 1.09 & 2.02 & 5.47 & 11.32 & 4.44 \\
Sharpe & 0.14 & 0.04 & 0.00 & 0.13 & 0.25 & 0.12 \\
\hline \hline
\end{tabular}

Average refers to the time series average of excess returns. The columns then provide the overall average (Mean), the cross-sectional standard deviation of the averages (St.dev), the minimum, median and maximum of the averages, and finally the average of the market excess return. The rows for Std dev provide the same cross-sectional information for the time series standard deviations and the individual Sharpe ratios, respectively. All returns are expressed in units of percent per month.

bivariate and trivariate sorts the linear combinations that are necessary to form near exact dependencies are more complex. Here we maintain both of these sorts along with the trivariate portfolios.

Except for the seven factors (five Fama-French plus Momentum and Long-Term Reversal), which are already excess returns, all series are expressed in excess of the risk-free rate. All returns are discrete time monthly returns. Summary statistics of the excess return data are in Table 2.2. A number of facts stand out. First, both the median and the mean average excess return of all included portfolios is above the average excess return of the market portfolio. The cross-sectional standard deviation (and min and max of the averages) indicate that there is a large dispersion in average returns. Second, most portfolios have a larger standard deviation than the marker portfolio, although some portfolios appear to have very low risk. Third, and important for our further results, there exist many portfolios that on their own already have a Sharpe ratio that is above the Sharpe ratio of the market portfolio. The maximum Sharpe ratio is for the portfolio of small firms with low prior volatility (ME_VAR_5x5_SMALL.LOVAR).

Table 2.3 contains our list of macroeconomic variables. The first macroeconomic variable is consumption. Consumption news is the most basic macroeconomic SDF factor. To measure news we first record consumption growth as the monthly change of log personal consumption expenditures. Our news variable is consumption growth in deviation of its long-term mean. When this is the only factor in the SDF its risk price measures the coefficient of relative risk aversion. Such a simple linear approximation may be poor, so an obvious extension is to have separate coefficients for positive and negative growth. These piecewise linear 
terms introduces some possible nonlinearities in the risk prices. We define the variable Negative Growth as the minimum of consumption growth and zero, which we then demean.

Table 2.3: Macro data sources

\begin{tabular}{ll}
\hline \hline $\begin{array}{l}\text { Personal Consump- } \\
\text { tion Expenditures }\end{array}$ & $\begin{array}{l}\text { US Bureau of Economic Analysis, Personal Consump- } \\
\text { tion Expenditures: Chain-type Price Index [PCEPI], } \\
\text { https://fred.stlouisfed.org/series/PCEPI }\end{array}$ \\
Consumer Price Index & $\begin{array}{l}\text { US Bureau of Labor Statistics, Consumer Price In- } \\
\text { dex for All Urban Consumers: All Items [CPIAUCSL], }\end{array}$ \\
& https://fred.stlouisfed.org/series/CPIAUCSL \\
Baa Corporate Bond & $\begin{array}{l}\text { Board of Governors of the Federal Reserve System, } \\
\text { Yield }\end{array}$ \\
& $\begin{array}{l}\text { Moody's Seasoned Baa Corporate Bond Yield [BAA], } \\
\text { https://fred.stlouisfed.org/series/BAA }\end{array}$ \\
$10-Y e a r$ & Board of Governors of the Federal Reserve Sys- \\
Rate & tem, 10-year treasury constant maturity rate [DGS10], \\
https://fred.stlouisfed.org/series/DGS10 & Federal Reserve Economic Data, Civilian Unemployment \\
Unemployment & Rate, https://fred.stlouisfed.org/series/UNRATE \\
\hline \hline
\end{tabular}

Other standard macroeconomic factors, at least since Chen et al. (1986), are inflation, credit spread, and term spread. In addition to these we also include unemployment as a business cycle indicator. To measure inflation we rely on the consumer price index of all urban consumers. The residuals of an $\operatorname{AR}(1)$ model for the monthly change in the log of the index provide our measure of unexpected inflation. Unemployment shocks are defined as the first difference of the unemployment rate. The change in the 10-year interest rate is used as a proxy for term structure news. For the credit spread we take the yield spread between the BAA rated yield and the 10/year government bond yield. Credit rate news are the residuals from an AR(1) filter. Summary statistics along with details of the construction of the news variables are reported in table 2.4.

Timing of information is not obvious for the macro series. With nowcasting and analyst expectations much of the news of current month data is already known before the end of the month. On the other hand, reported data and revisions imply that data for month $t$ are often only known at a later date. To minimize the impact of timing uncertainty on the construction of the tracking portfolios, we use the sum of the news at times $t-1, t$ and $t+1$ as the dependent variable $x_{t}$ 
Table 2.4: Summary statistics macro variables

\begin{tabular}{lccc}
\hline \hline Series & St. Dev. & Skewness & Kurtosis \\
\hline Consumption $(\Delta c)$ & 0.502 & 0.13 & 6.15 \\
Negative Growth $(\min (0, \Delta c))$ & 0.553 & -2.04 & 8.88 \\
Inflation $((1-\phi \mathrm{L}) \Delta p)$ & 0.251 & 0.06 & 8.16 \\
Unemployment $(\Delta U)$ & 0.356 & 1.43 & 7.31 \\
Negative Unemployment $(\min (0, \Delta U))$ & 0.170 & -1.20 & 5.21 \\
Long-Term Interest rate $(\Delta R)$ & 0.338 & -0.28 & 6.15 \\
Credit Spread $((1-\phi \mathrm{L}) C)$ & 0.223 & 0.61 & 9.35 \\
\hline Statistics refer to monthly data for January 1965 to February 2018. The news filter for \\
the data is indicated in parentheses: $\Delta$ means first differencing, $\phi \mathrm{L}$ indicated an AR $(1)$ \\
adjustment. All series are demeaned. Filters are run on the complete data series, extending \\
to the earliest available date in the source. Units are percent per month.
\end{tabular}

for the all macro variables except the Credit Spread and the Long-Term Interest Rate.

\section{$2.6 \quad$ Results}

\subsubsection{Tracking portfolios}

Crucial for estimating the risk prices are the first stage projections of the macroeconomic news variables on the space of excess returns. Table 2.5 provides summary statistics of the tracking portfolios. For all series the BIC criterion selects a much larger model than cross-validation. As a consequence the BIC portfolios also have a larger standard deviation. Most of this is by construction, since every iteration adds to the variance of the fitted returns.

As we have just a small set of macro variables, we will discuss variables individually. Starting with Consumption, the projection more or less delivers what we would expect. Consumption is related to returns, but the correlation is not that large, even when we allow searching among a large set of portfolios. Using cross validation the optimal tracking portfolio explains about $8 \%$ of the variation in the consumption shocks. Using the BIC criterion the algorithm continues much longer, and generates a tracking portfolio with an $R^{2}$ of $19 \%$. As with most tracking portfolios the correlation between the two alternatives, termination by CV versus the BIC stopping rule, is large (see last column of Table 2.5). Figure 2.1 shows how the fit of the model evolves with the number of boosting iterations. The fit of the model remains fairly stable as the iterations progress. Despite the large number 
Table 2.5: Tracking portfolio summary statistics

\begin{tabular}{|c|c|c|c|c|c|c|c|c|c|}
\hline & \multicolumn{4}{|c|}{$\mathrm{CV}$} & \multicolumn{4}{|c|}{$\mathrm{BIC}$} & \multirow[b]{2}{*}{$\rho$} \\
\hline & $R^{2}$ & $\operatorname{trH}$ & SDev & $\mathrm{Sh}$ & $R^{2}$ & $\operatorname{trH}$ & SDev & Sh & \\
\hline Consumption & 0.08 & 1.0 & 0.13 & 0.07 & 0.19 & 9.0 & 0.21 & 0.07 & 0.88 \\
\hline Neg. growth & 0.04 & 1.1 & 0.06 & 0.12 & 0.28 & 18.2 & 0.15 & 0.08 & 0.61 \\
\hline Inflation & 0.22 & 8.2 & 0.13 & 0.04 & 0.43 & 29.4 & 0.20 & 0.13 & 0.89 \\
\hline Long-term rate & 0.44 & 20.1 & 0.17 & 0.03 & 0.49 & 28.8 & 0.18 & 0.03 & 0.99 \\
\hline Credit spread & 0.29 & 9.5 & 0.08 & 0.12 & 0.39 & 20.0 & 0.10 & 0.10 & 0.95 \\
\hline Unemployment & 0 & 0 & 0 & 0 & 0.31 & 19.7 & 0.10 & 0.01 & 0 \\
\hline Neg. unemp. & 0.07 & 3.3 & 0.02 & 0.14 & 0.18 & 11.7 & 0.04 & 0.23 & 0.80 \\
\hline
\end{tabular}

The table shows summary statistics for the tracking portfolios constructing using the $L_{2}$ boosting algorithm. $R^{2}$ is the squared correlation between the macro news and the tracking portfolio; trH is Bühlmann's degrees of freedom parameter $k$ defined in (2.22); SDev is the standard deviation of the tracking portfolio excess returns; Sh is the Sharpe ratio of the tracking portfolio excess returns. For the left side of the table boosting iterations are terminated by minimizing the test error sum of squares using 5 -fold cross-validation (CV). For the right-side of the table the stopping criterion is based on the BIC. In the final column $\rho$ is the correlation between the returns of the BIC and CV tracking portfolios. Abbreviations: Neg. growth $=\min (0, \Delta c)$, Neg. Unemp $=\min (0, \Delta U)$.

of additional steps needed to minimize the BIC criterion, the algorithm does not make much progress in fitting the consumption growth. The correlation seems sufficiently large to provide a valid instrument; yet it remains low enough not to raise suspicion of overfitting.

Figure 2.2 shows the evolution of the tracking portfolio as a function of the number of boosting iterations. The most highly correlated portfolio is the sort on firms with negative cashflow-to-price $\left(\mathrm{CF}-\mathrm{P}_{-}<=0\right)$. This is also the portfolio that in the end receives the largest weight in the consumption tracking portfolio. At later steps the algorithms starts to increase the coefficient on Automobile Industry (49_Industry_Autos) to make it the second most important component of the tracking portfolio based on the BIC stopping criterion.

Most remarkable is the low Sharpe ratio of the tracking portfolio. Whether we use CV or BIC as the stopping criterion, the tracking portfolio has a Sharpe ratio that is much below the Sharpe ratio of the market portfolio, and is in the lower part of the distribution over all the portfolio sorts in the database. Since the consumption tracking portfolio apparently combines assets in a way that leads to a low Sharpe ratio, it will never be able to explain the high Sharpe ratio of a mean-variance efficient portfolio. Partly this is known as the equity premium puzzle, but here it comes as more of a surprise, since the algorithm could choose from many candidate portfolios, most of which have Sharpe ratios that are above 
Figure 2.1: Tracking portfolio fit
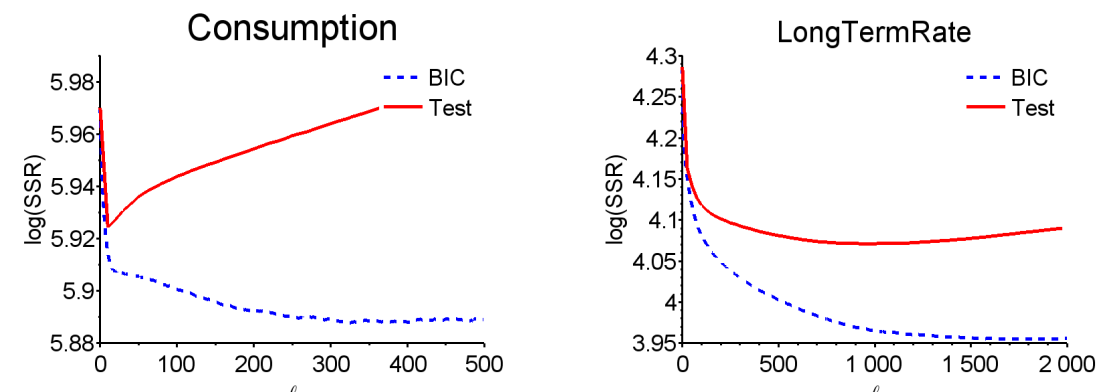

The figure shows the fit of the tracking portfolio for macroeconomic ${ }^{\ell}$ news as a function of the number of boosting steps $(\ell)$. The solid line Test refers to the sum of squared residuals (SSR) of the 5 -fold cross-validation as is plotted as $\ln (\mathrm{SSR})$. The dashed line is the BIC criterion $\ln (\mathrm{SSR})+k \ln T / T$, where $k$ is the model complexity and $T$ the time series sample size. Without any regressor $(\ell=0)$ the two criteria are both equal to the log sum of squares $\sum_{t} x_{t}^{2}$ of the news series.

that of the market. Diversifying over these portfolios would easily enable much larger Sharpe ratios.

For Negative Consumption Growth the tracking portfolio results are qualitatively similar. Cross-validation identifies a very sparse model, this time consisting mostly of the Automobile industry. The BIC criterion again selects a more complex model. The most important additional asset selected by BIC is one of the low volatility portfolios (ME_VAR_5x5_ME3.VAR1). Since this portfolio is added at a later stage during the model selection, the correlation between the CV and BIC stopped tracking portfolio returns is lower than for most other factors. When Negative Growth is included in our later SDF pricing regressions we may thus expect some sensitivity with respect to which stopping criterion we use.

Among the macro news variables Inflation appears to be the easiest to fit. The CV stopping criterion already identified a large model with an $R^{2}$ of $22 \%$. The most important weight is the Oil industry (49_Industry_0il) followed by Coal (49_Industry_Coal).

The most puzzling results occur for Unemployment. Here cross-validation does not find any return that correlates with the macro news series. What this means is that if we randomly select $80 \%$ of the months in the sample and run the boosting algorithm on this sample to construct tracking portfolio weights, the out-of-sample fit on the remaining $20 \%$ of the data is worse than having no model at all. The BIC criterion, however, using the full sample both for fitting and evaluation, does find substantial explanatory power. The discrepancy between the two is most 
Figure 2.2: Tracking portfolio composition

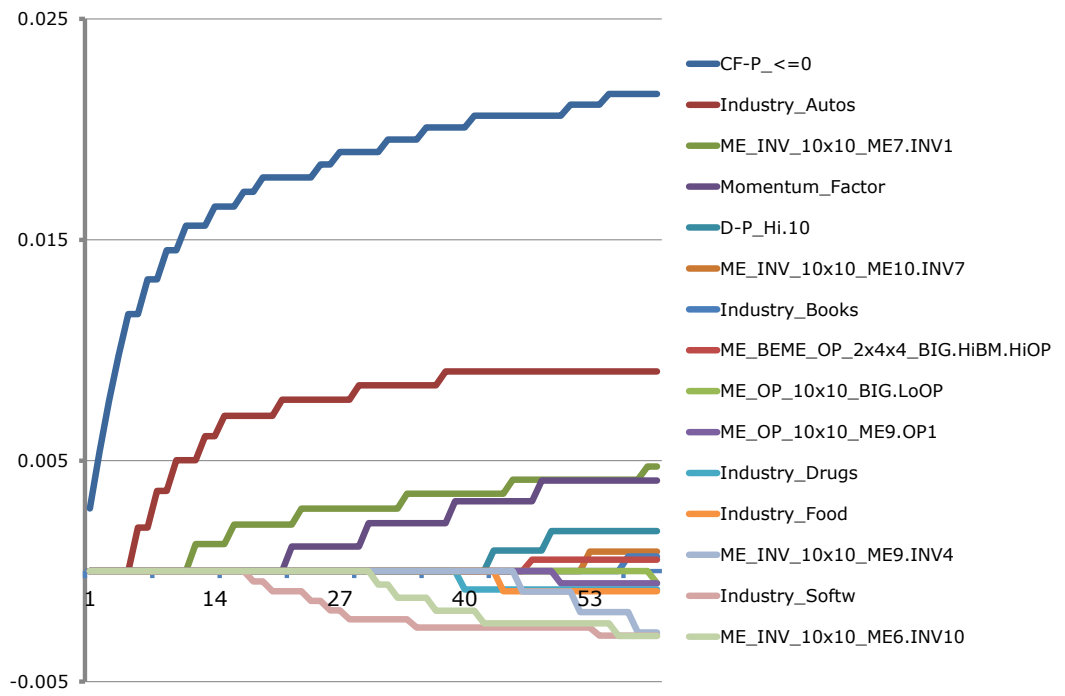

(a) Consumption

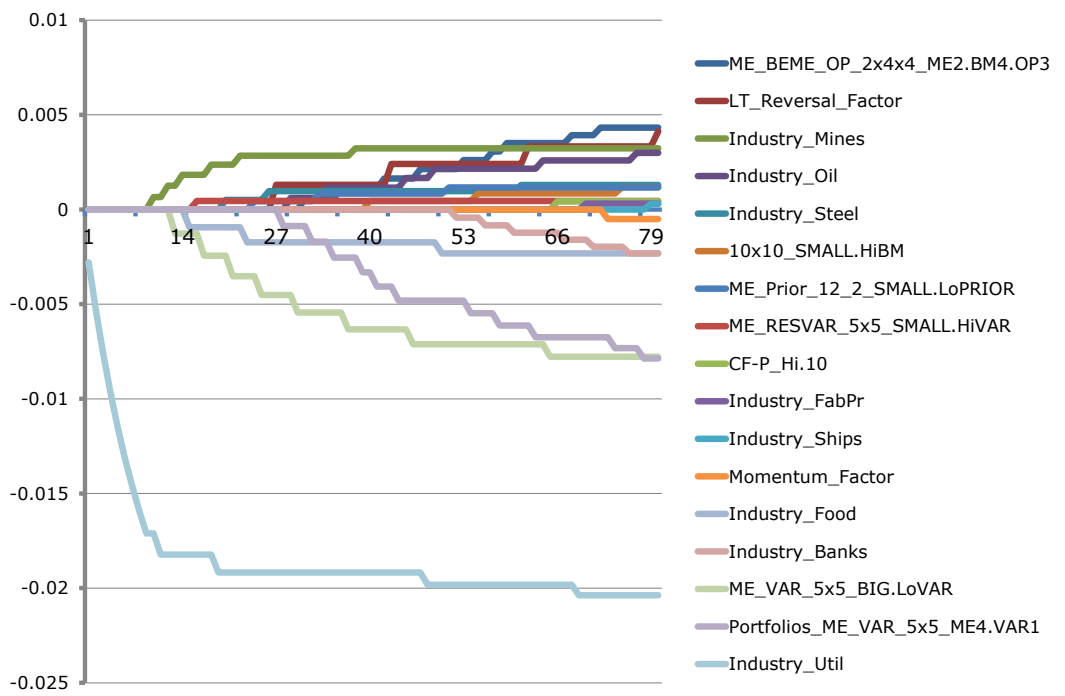

(b) Long-term interest rate

The figure shows the evolution of the tracking portfolio weights as a function of the number of iterations of the $L_{2}$-boosting algorithm. At each step the algorithm adjusts the coefficient of the return portfolio that has the highest correlation with the current residuals. 
Figure 2.3: Tracking portfolio returns
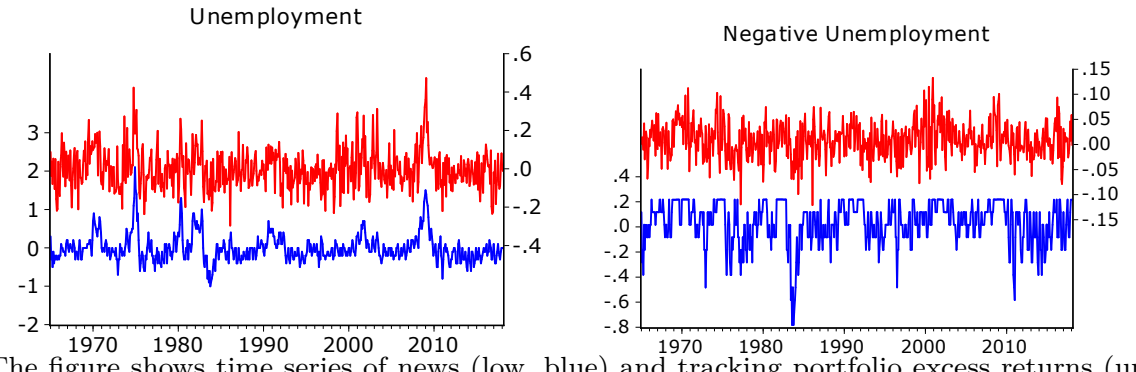

The figure shows time series of news (low, blue) and tracking portfolio excess returns (up, red) for the Unemployment and Negative Unemployment data. The tracking portfolio uses the regression fit identified by the BIC criterion.

likely related to outliers that happen to coincide in both the news series and in some return portfolio. With full sample estimation these outliers are influential, whereas they will often be absent in randomly selected subsamples used by cross validation. Figure 2.3 shows that some of this can indeed explain the big difference between BIC and CV in this case. In the full sample the two biggest increases in unemployment (in 1975 and 2009) are fitted reasonably well by the BIC tracking portfolio.

Negative unemployment does not have the positive outliers and is easier to track with cross validation. By its definition a shock to negative unemployment is a decrease in unemployment, and thus positive economic news. If strong 'negative' news would be priced, the complement $U^{+}=U-U^{-}$will be priced in the SDF, leading to opposite coefficients for $U$ and $U^{-}$. Anticipating the factor pricing regressions, it is important to note that the tracking portfolio for Negative unemployment attains the highest Sharpe ratio among all tracking portfolios, both according to $\mathrm{CV}$ as well as BIC.

The two financial factors, Credit Spread and Long-term interest rate are less subject to measurement error and timing of news. Cross validation already fits both with high $R^{2}$ 's. Even though the BIC models are a bit less sparse, the tracking portfolios are very similar under BIC and CV. For both factors the BIC and $\mathrm{CV}$ returns are almost perfectly correlated. One of the more noticeable properties of the interest rate tracking portfolio is the large (negative) weight for the utilities industry (49_Industry_Util). The evolution of the portfolio weights is shown in Figure 2.2. The tracking portfolio for the credit spread is dominated by 
Table 2.6: Discount factor model estimates

\begin{tabular}{|c|c|c|c|c|c|c|c|c|}
\hline & \multicolumn{4}{|c|}{$\mathrm{CV}$} & \multicolumn{4}{|c|}{$\mathrm{BIC}$} \\
\hline & CAPM & $\mathrm{C}+$ & macro & all & CAPM & $\mathrm{C}+$ & macro & all \\
\hline Consumption & $\begin{array}{c}0.32 \\
(1.7)\end{array}$ & $\begin{array}{l}-0.51 \\
(1.0)\end{array}$ & $\begin{array}{c}0.54 \\
(0.2)\end{array}$ & $\begin{array}{l}-0.61 \\
(1.0)\end{array}$ & $\begin{array}{r}0.21 \\
(1.7)\end{array}$ & $\begin{array}{c}0.19 \\
(0.9)\end{array}$ & $\begin{array}{c}0.16 \\
(1.1)\end{array}$ & $\begin{array}{r}0.18 \\
(0.8)\end{array}$ \\
\hline Neg. growth & & $\begin{array}{c}1.91 \\
(1.9)\end{array}$ & & $\begin{array}{r}2.26 \\
(1.6)\end{array}$ & & $\begin{array}{c}0.05 \\
(0.2)\end{array}$ & & $\begin{array}{l}-0.12 \\
(0.4)\end{array}$ \\
\hline Inflation & & & $\begin{array}{c}0.30 \\
(1.2)\end{array}$ & $\begin{array}{c}0.56 \\
(1.6)\end{array}$ & & & $\begin{array}{r}0.57 \\
(3.8)\end{array}$ & $\begin{array}{r}0.39 \\
(2.3)\end{array}$ \\
\hline Long-term rate & & & $\begin{array}{c}0.12 \\
(0.4)\end{array}$ & $\begin{array}{l}-0.31 \\
(0.7)\end{array}$ & & & $\begin{array}{l}-0.13 \\
(0.6)\end{array}$ & $\begin{array}{l}-0.06 \\
(0.3)\end{array}$ \\
\hline Credit spread & & & $\begin{array}{l}-1.22 \\
(2.0)\end{array}$ & $\begin{array}{l}-0.12 \\
(2.40)\end{array}$ & & & $\begin{array}{l}-0.71 \\
(2.0)\end{array}$ & $\begin{array}{l}-0.82 \\
(2.0)\end{array}$ \\
\hline Unemployment & & & & & & & $\begin{array}{l}-0.12 \\
(0.4)\end{array}$ & $\begin{array}{l}-2.40 \\
(4.9)\end{array}$ \\
\hline Neg. unemp. & & & & $\begin{array}{c}3.62 \\
(2.8)\end{array}$ & & & & $\begin{array}{c}6.53 \\
(6.2)\end{array}$ \\
\hline
\end{tabular}

The table reports estimates of the risk prices $\delta$ in the SDF model $m=1-\delta^{\prime} x$ using instrumental variables $\hat{x}$. The instrument for macroeconomic variable $x_{j}$ are the tracking portfolio returns $\hat{x}_{j}$. The left side of the table uses instruments based on cross-validation (CV); the right side of the table is based on the BIC for instrument selection. In parentheses we report t-statistics.

various low volatility portfolios (ME_VAR_5x5_ME3.VAR1, ME_VAR_5x5_ME2.VAR1, ME_VAR_5x5_ME4.VAR1)

\subsubsection{Pricing kernel estimates}

The tracking portfolios serve as instruments for the macroeconomic news when estimating the SDF equation (2.7) using the IV estimator (2.9) and standard errors as in (2.11). Results are in Table 2.6, for both the CV and BIC tracking portfolios.

Consistent with most of the empirical literature the basic Consumption CAPM with only consumption growth as a factor is not very successful. The price of consumption risk is barely significant. ${ }^{2}$ More important are the time series properties of the SDF time series. The actual SDF, i.e. the regression residuals $\hat{m}_{t}=1-\hat{\delta} \Delta c_{t}$,

\footnotetext{
${ }^{2}$ Due to the scaling of the variables, the coefficient itself (0.32) must be multiplied by 100 to interpret it as the coefficient of relative risk aversion. An estimate around 32 is not unusual, and may be even be on the low side. The consumption series used in the IV estimation as well as in the SDF construction is the sum of the time $t-1, t, t+1$ shocks, consistent with the
} 
is highly volatile, since consumption news has a standard deviation of 0.78 leading to pricing kernel volatility of $0.32 \times 0.78=0.25$. Even though the pricing kernel is only identified up to a scalar multiple (since we work with excess returns), the volatility of $m$ is still the maximum Sharpe ratio for any portfolio. The consumption factor could thus account for a monthly Sharpe ratio of 0.25. However, that portfolio must be perfectly correlated with consumption. By definition the maximally correlated portfolio is the tracking portfolio, which only has a correlation of $0.076^{2}=0.28$ (see Table 2.5). More relevant for pricing, therefore, is the projection on the space of asset returns. For this we construct $u_{t}=1-\hat{\delta} \hat{x}_{t}^{C}$, with $\hat{x}_{t}^{C}$ the tracking portfolio excess return. The tracking portfolio has a standard deviation of 0.125 , which gives a volatility of only 0.04 for the projected SDF, and therefore a very low maximum Sharpe ratio. Consistent with the equity premium literature consumption as a single factor cannot explain high Sharpe ratios. Using the BIC estimate for $\delta(0.21)$ and the standard deviation for the BIC tracking portfolio (0.213) leads to the same volatility for the projected SDF. The difference in the estimates for $\delta$ between $\mathrm{CV}$ and BIC is thus consistent with the different volatilities of the tracking portfolios. ${ }^{3}$

Adding negative consumption growth to the SDF specification leads to different results for $\mathrm{CV}$ and $\mathrm{BIC}$ instruments. For the $\mathrm{CV}$ tracking portfolio the nonlinearity in consumption growth is almost significant and greatly increases the volatility of the discount factor and its ability to generate high Sharpe ratios. The importance of the negative growth variable is related to the high Sharpe ratio of its tracking portfolio. ${ }^{4}$ With the BIC instrument selection the tracking portfolio for negative growth loses its high Sharpe ratio (Table 2.5). Hence the estimate for its risk price becomes much smaller and insignificant.

After the detailed discussion of the consumption risk prices, the remainder of Table 2.6 is almost self explanatory. We find large t-statistics for risk prices for those variables that have a tracking portfolio with a high Sharpe ratio. For the

dependent variable in the tracking portfolio construction. This adds roughly another factor $\sqrt{3}$ to the scaling and would bring the CRR estimate up to 55 .

3 Technically, the IV estimator for $\delta$ in the single factor model equals $\hat{\delta}=\mathrm{E}[\hat{x}] / \operatorname{cov}[x, \hat{x}]$, which implies that the volatility of the SDF satisfies $\rho_{x, \hat{x}} s(m)=\mathrm{E}[\hat{x}] / s(\hat{x})$. The volatility of the SDF is directly related to the Sharpe ratio of the tracking portfolio, with a proportionality constant equal to the correlation between $x$ and its tracking portfolio $\hat{x}$.

4 In a multivariate IV regression the relation between the discount factor volatility and the tracking portfolio Sharpe ratios is not as clear cut as in the univariate regression due to the correlations among tracking portfolios. Under the IV estimator the SDF variance is still a quadratic form in the average returns of the tracking portfolios: $s^{2}(m)=$ $\mathrm{E}[\hat{x}]^{\prime} \mathrm{E}\left[\hat{x} x^{\prime}\right]^{-1} \mathrm{E}\left[x x^{\prime}\right] \mathrm{E}\left[x \hat{x}^{\prime}\right]^{-1} \mathrm{E}[\hat{x}]$ Since $\mathrm{E}[x]=0$ by assumption, the second moments are covariances. 
Table 2.7: Mean-Variance tracking portfolio

\begin{tabular}{|c|c|c|c|c|c|c|c|c|c|}
\hline & \multicolumn{4}{|c|}{$\mathrm{CV}$} & \multicolumn{4}{|c|}{$\mathrm{BIC}$} & \multirow[b]{2}{*}{$\rho$} \\
\hline & RMSE & $\operatorname{trH}$ & SDev & $\mathrm{Sh}$ & RMSE & $\operatorname{trH}$ & SDev & Sh & \\
\hline MV & 0.56 & 71.0 & 0.35 & 1.69 & 0.56 & 60.5 & 0.36 & 1.58 & .99 \\
\hline \multicolumn{10}{|c|}{$\begin{array}{l}\text { The table shows summary statistics for the mean-variance tracking portfolio constructed } \\
\text { using the } L_{2} \text {-boosting algorithm. RMSE is the root-mean-squared-error of the regression } \\
\text { residuals; trH is Bühlmann's degrees of freedom parameter } k \text { defined in }(2.22) \text {; SDev is the } \\
\text { standard deviation of the tracking portfolio excess returns; Sh is its Sharpe ratio. For the } \\
\text { left side of the table boosting iterations are terminated by minimizing the test error sum of } \\
\text { squares using } 5 \text {-fold cross-validation. For the right-side of the table the stopping criterion } \\
\text { is based on the BIC. In the final column } \rho \text { is the correlation between the returns of the BIC } \\
\text { and CV tracking portfolios. }\end{array}$} \\
\hline
\end{tabular}

CV based instruments the significant risk prices are associated with the Credit spread and Negative unemployment; for the BIC instruments Inflation and Unemployment are significant as well. The latter is the exception to the rule, since the Sharpe ratio for the unemployment tracking portfolio is very low. In the multiple regression it is significant due to its correlation with the tracking portfolio for negative unemployment. The two prices have opposite signs, indicating that it is mostly increases in unemployment that are priced.

\subsubsection{Mean-Variance efficient portfolio}

For further assessments of the ability of the multivariate SDF models for pricing the cross-section we need the Hansen-Jagannathan distance. First we construct a mean-variance efficient portfolio by projecting a vector of ones on the space of excess returns. Figure 2.4 shows how the fit of the MV tracking portfolio evolves with the number of boosting steps. Both $\mathrm{CV}$ and BIC require many more steps than for any of the macro tracking portfolios. The two criteria produce almost identical portfolios with a correlation above 0.99. The tracking portfolio is very well diversified. It uses 192 out of the 809 available portfolios. ${ }^{5}$ The largest absolute weight of a single constituent of the tracking portfolio is only 0.035 , with an average absolute weight of 0.012 .

The big challenge to any asset pricing model is the huge Sharpe ratio of the tracking portfolio. Its value (in monthly units) is more than 1.5, more than 6 times as large as the largest single portfolio Sharpe ratio in the database. ${ }^{6}$ The

\footnotetext{
${ }^{5}$ Recall that to avoid near arbitrage about 100 redundant portfolios are deleted when fitting the mean-variance portfolio, see section 2.5)

6 This is the portfolio ME_VAR_5x5_SMALL.LoVAR.
} 
tracking portfolio loads heavily on this low-volatility portfolio. Its counterpart, a portfolio with high residual volatility (ME_RESVAR_5x5_SMALL.HiVAR) obtains an equally negative weight. In addition there are large positive weights for some of the momentum and reversal portfolios (ME_Prior_1_0_SMALL.LOPRIOR) and the (ME_Prior_12_2_SMALL.HiPRIOR) as well as large negative weights for Size/BM and reversal portfolios (10x10_SMALL.LOBM) and (ME_Prior_1_0_SMALL.HiPRIOR). Typically, many of the small stock portfolios are selected. One of the more remarkable results is that the seven factor portfolios (5 Fama-French plus Momentum and Reversal) do not obtain strong weights. In fact, the original three FF factors (Market, SMB, HML) are not selected at all and therefore have zero weight. Only the CMA and Momentum factor have a weight close to the average absolute weight.

The huge Sharpe ratio almost surely overestimates the true Sharpe ratio of the MV-portfolio. A clear indication for this is the Sharpe ratio obtained in test samples using cross validation. The graph on the right in Figure 2.4 shows the Sharpe ratio ('Test') with increasing model complexity. For the test samples the portfolio weights are estimated on a random subsample with $80 \%$ of the observations, and the Sharpe ratio computed on the fitted portfolio returns in the remaining $20 \%$. This out-of-sample Sharpe ratio is extremely stable around 0.55 irrespective of the model complexity. While still a large Sharpe ratio, it is not anywhere near the insample estimate when portfolio weights and performance measures are computed on the same sample.

For the purpose of optimal-portfolio construction the MV portfolio may not be optimal due to the unconstrained nature of its composition. Our interest here is to construct a benchmark for measuring the fit of asset pricing models. As such the MV portfolio generates a time series of excess returns on the mean-variance frontier.

\subsubsection{Asset pricing}

Knowing the properties of the mean-variance efficient portfolio we can compute the Hansen-Jagannathan distance to evaluate how well the alternative specifications perform. Due to the huge Sharpe ratio of the MV-portfolio it is clear that none of the models can fully explain the cross-section. The absolute values of the $H J$ distance may therefore not be very meaningful. Their relative magnitudes still provide an interesting comparison of the different models. 
Figure 2.4: Efficient frontier estimates
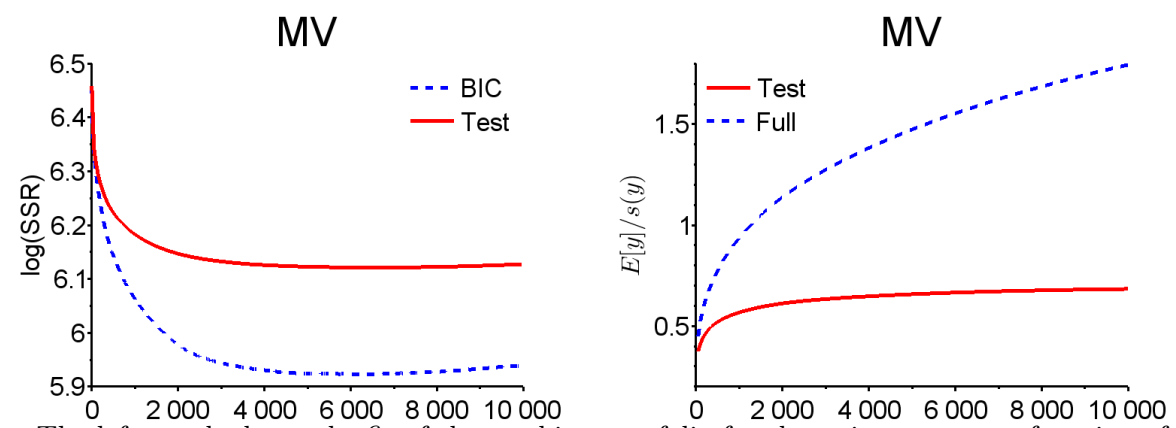

The left graph shows the fit of the tracking portfolio for the unit vector as a function of the number of boosting steps. The solid line Test refers to log of the sum of squared residuals $\log (\mathrm{SSR})$ of the 5 -fold cross-validation. The dashed line is the BIC criterion. The graph on the right compares the Sharpe ratio of the fitted values in test samples with results for the full sample. A complete test sample is defined as the $T$ observations from all $K$ segments of a single cross-validation. The Sharpe ratio is defined as the (absolute) average return divided by its standard deviation. Test sample results are obtained by averaging over 100 cross-validations. Returns are expressed in percent per month.

From the results in Table 2.8 we learn that the BIC instruments always fit better than their $\mathrm{CV}$ counterparts. With the $\mathrm{CV}$ portfolios, adding all variables in the SDF model only leads to a modest $8 \%$ decrease in the $H J$ distance relative to the CAPM. This indicates that the additional macro factors do not improve much on the consumption based model. The only substantial improvement occurs when all factors are included and instrumented through the BIC. Because of the large Sharpe ratio associated with the nonlinear unemployment effect, the $H J$ distance improves with nearly $20 \%$.

Table 2.8: Hansen-Jagannathan distance

\begin{tabular}{lcccc}
\hline \hline & CAPM & C + & macro & all \\
\hline Cross Validation & 0.468 & 0.458 & 0.454 & 0.430 \\
BIC & 0.439 & 0.436 & 0.415 & 0.360 \\
\hline
\end{tabular}

For each of the SDF models in table 2.6 the Hansen-Jagannathan distance is computed as $H J=\hat{u}^{\prime} \hat{u} / T$ where $\hat{u}=\hat{1}-\hat{\boldsymbol{X}} \hat{\delta}$ are residuals of the MV-tracking portfolio returns with respect to the tracking portfolio returns.

As a final test of the factor tracking portfolios, and the importance of the macroeconomic factors, we consider their weight in approximating the meanvariance efficient portfolio. To do so we consider the spanning regression (2.14) 
with an additional intercept to measure the alpha of the MV portfolio relative to our factor portfolios. If the factors fully explain the cross-section the regression should fit perfectly and the intercept should be zero. What we observe in Table 2.9, as expected, are large highly significant intercepts at the size of more than half a percent per month. Again, this relates to the low Sharpe ratios of the macro tracking portfolios. Combining these macro factors does not bring enough diversification benefits to come near the performance of the boosted mean-variance efficient portfolio.

Table 2.9: Spanning regressions

\begin{tabular}{|c|c|c|c|c|c|c|c|c|}
\hline & \multicolumn{4}{|c|}{$\mathrm{CV}$} & \multicolumn{4}{|c|}{$\mathrm{BIC}$} \\
\hline & CAPM & $\mathrm{C}+$ & macro & all & CAPM & $\mathrm{C}+$ & macro & all \\
\hline Consumption & $\begin{array}{c}0.25 \\
(1.7)\end{array}$ & $\begin{array}{l}-0.34 \\
(1.5)\end{array}$ & $\begin{array}{c}0.09 \\
(0.7)\end{array}$ & $\begin{array}{l}-0.56 \\
(2.4)\end{array}$ & $\begin{array}{c}0.24 \\
(3.7)\end{array}$ & $\begin{array}{r}0.11 \\
(1.2)\end{array}$ & $\begin{array}{c}0.22 \\
(2.8)\end{array}$ & $\begin{array}{c}0.11 \\
(1.2)\end{array}$ \\
\hline Neg. growth & & $\begin{array}{c}1.52 \\
(2.9)\end{array}$ & & $\begin{array}{c}1.83 \\
(3.3)\end{array}$ & & $\begin{array}{c}0.28 \\
(2.2)\end{array}$ & & $\begin{array}{c}0.32 \\
(2.4)\end{array}$ \\
\hline Inflation & & & $\begin{array}{c}0.19 \\
(1.7)\end{array}$ & $\begin{array}{r}0.27 \\
(2.4)\end{array}$ & & & $\begin{array}{c}0.29 \\
(3.9)\end{array}$ & $\begin{array}{r}0.31 \\
(4.3)\end{array}$ \\
\hline Long-term rate & & & $\begin{array}{l}-0.11 \\
(1.0)\end{array}$ & $\begin{array}{c}-0.12 \\
(1.1)\end{array}$ & & & $\begin{array}{l}-0.24 \\
(2.4)\end{array}$ & $\begin{array}{l}-0.26 \\
(2.7)\end{array}$ \\
\hline Credit spread & & & $\begin{array}{c}-0.50 \\
(1.9)\end{array}$ & $\begin{array}{c}-0.29 \\
(1.1)\end{array}$ & & & $\begin{array}{c}-0.19 \\
(0.9)\end{array}$ & $\begin{array}{c}-0.08 \\
(0.4)\end{array}$ \\
\hline Unemployment & & & & & & & $\begin{array}{l}-0.05 \\
(0.4)\end{array}$ & $\begin{array}{l}-0.51 \\
(2.7)\end{array}$ \\
\hline Neg. unemp. & & & & $\begin{array}{r}2.31 \\
(3.0)\end{array}$ & & & & $\begin{array}{c}6.53 \\
(6.2)\end{array}$ \\
\hline$\alpha$ & 0.59 & 0.58 & 0.58 & 0.57 & 0.56 & 0.56 & 0.55 & 0.53 \\
\hline$\hat{\sigma}$ & 0.35 & 0.35 & 0.35 & 0.35 & 0.35 & 0.35 & 0.35 & 0.34 \\
\hline
\end{tabular}

Despite the high alpha's, the regressions offer some insights in the potential of macro factors. Consumption matters. Both on its own, as well as with a nonlinear effect added, the consumption tracking portfolios explain part of the return on the MV-portfolio. It is especially the nonlinearity, which emphasizes negative consumption growth, that contributes significantly to the return of the MV portfolio. 
The same nonlinearity also makes unemployment an important contributor. Here the nonlinearity is even more important due the high Sharpe ratio of its tracking portfolio. Inflation and the long-term interest rate are significant as well.

\subsection{Conclusion}

In this chapter we address the big- $N$ problem of testing asset pricing moment conditions when their is a large number of test assets but a few short time series of macroeconomic factors. In order to assess the fit and significance of a given macroeconomic risk factor model we reinterpret the Hansen-Jagannathan distance as the residual variance of a time-series instrumental variables regression, where the number of instruments is large. Guided by theoretical results in Belloni et al. (2012), we propose the $L_{2}$-boosting algorithm to solve the high-dimensional instrument selection problem. This algorithm weights test assets by their correlation with the risk factor and aggregates them into a tracking portfolio that serves as a strong instrument. The algorithm is equipped to deal with applications where the number of assets is large relative to the number of time series observations.

We implement our method to assess standard macro economic factors like consumption, inflation and industrial production. To form tracking portfolios for these factors we use around 900 financial portfolios from Kenneth French database. We find that the pricing kernels based on macro economic risk factors generally leave much of the variance of the efficient frontier unexplained. However, despite the low fit we find that classic factors such as consumption growth and inflation are priced.

Several issues remain. First, since the few standard macro factors do not explain the cross-section, there is obviously of interest to extend the factor space to alternative macro factors. Both on the monthly, and especially at the quarterly, frequency many more macro factors are available and theoretically potentially relevant. The number of factors will quickly proliferate when we also allow interactions and nonlinearities. From our limited experience in this chapter, nonlinearities may prove important. As more macro factors are introduced, there will be a need for a second learning procedure for selecting the most promising factors or transformations and combinations of factors. Our focus in this chapter has been on the big- $N$ aspect. When $M$ and $N$ can both be large, there is an interesting double statistical learning element to the model selection. 
The second main issue is the construction of the high-dimensional mean-variance portfolio. While not needed for estimating the risk prices, it is the benchmark for evaluating the fit of the stochastic discount factor model. Constructing MVportfolios is a notoriously difficult problem, for which the $L_{2}$-boosting is an interesting new approach, but its properties have not been fully explored in this chapter. The full sample mean-variance efficient portfolio that we obtained from the $L_{2}$-boosting algorithm seems a poor benchmark for testing the asset pricing implications. Its huge in-sample Sharpe ratio, many times bigger than any of the macro factor tracking portfolios, implies that none of our models has a chance to fit very well. On the other hand, since the Sharpe ratio on cross-validation samples is much lower, it may be more appropriate to compute the Hansen-Jagannathan distance and spanning alpha's on cross validation samples. 



\section{Chapter 3}

\section{Optimizing large portfolios using factors and sparse hedging}

We use a combination of financial factors and sparse hedging portfolios to allocate a large number of assets into a minimum variance portfolio. Regularized hedging portfolios can be formed using the graphical lasso but relies on statistical sparsity assumptions. The factors serve an important role to make these assumptions more suitable. In turn, the estimated hedging portfolios complement the factor model by allowing for deviations from the strict factor structure. We differ from previous methods in that this approach allows us to circumvent the explicit high-dimensional matrix inversions. Empirically we find that our method reduces portfolio volatility more than competing methods, especially when the number of assets far exceed the amount of historical returns. ${ }^{1}$

\footnotetext{
${ }^{1}$ This chapter is based on the paper Lönn (2018).
} 


\subsection{Introduction}

We present an approach to minimum-variance portfolio allocation that combines high-dimensional model selection and common factor models. This approach is especially well suited to handle the situation where the number of assets is large relative to the length of the time series. The main empirical challenge is that the allocation relies on an estimate of the inverse of a large covariance matrix, which is typically very poorly estimated. A crucial part of our solution includes the graphical lasso (Yuan and Lin, 2007; Friedman et al., 2008), which estimates the inverse directly. However, the estimator requires that we assume that many elements of the inverse are zero, this is our sparsity assumption. To motivate this assumption we introduce a common factor structure for the financial returns. Using the graphical lasso conditional on the factor model we allow for returns to deviate from the strict factor structure. We depart from previous works in the financial and statistical literature by emphasizing the conditionally sparse inverse covariance matrix. We demonstrate that this framework can provide a compelling approach when expected returns are subject to approximate clusters, for example along the lines of industry. Empirically, we find that our Factor-Graphical method consistently reduces volatility more than competing methods. Our method many times produce portfolio volatilities that are between ten and fifteen percent lower than the second best performing method.

Mean-variance optimal portfolios are an enduring empirical puzzle where theoretically optimal allocations often perform very poorly out-of-sample. ${ }^{2}$ When the number of assets $N$ is large the crucial challenge is estimating the covariance matrix of returns (Kan and Zhou, 2007). In these large- $N$ allocations the number of parameters to estimate in the covariance matrix is vast, $N(N+1) / 2$. In addition the allocation rule also requires that the $\operatorname{large}-N$ covariance matrix be inverted. There are several methods that approach these issues. Methods such as the Shrinkage estimators (Ledoit and Wolf, 2003, 2004, 2012) and the POET estimator (Fan, Liao, and Mincheva, 2013) makes use of factor structures to improve the covariance estimation. Alternatively, Goto and Xu (2015) use the graphical lasso to circumvent the covariance estimation and directly estimate the inverse. Our method combines the graphical methodology with financial factor structure.

\footnotetext{
${ }^{2}$ There is an extensive literature that document this but among the foremost are Michaud (1989) and DeMiguel et al. (2007)
} 
The method we propose makes use of two assumptions. First, a parsimonious model of common factors describes the commonality of returns. Second, accounting for those factors the inverse covariance matrix of returns is sparse. The second assumption implies that conditional on a factor model, only a small number of off-diagonal elements in the inverse covariance matrix are non-zero. Rephrasing this constraint from a regression perspective clarifies the financial rationale. Let $y_{i}$ denote the return on asset $i$, then the row/column $i$ of the inverse covariance matrix is proportional to the negative regression parameters of $y_{i}=\sum_{j \neq i} y_{j} \gamma_{j}+\varepsilon_{i}$. Stevens (1998) shows that the scaled negative regression coefficients correspond to the optimal hedging weights for the dependent asset $y_{i}$, e.g. a long position in asset $i$ is optimally hedged by a short position in $j$ proportional to $\gamma_{j}$. Thus, each row/column of the inverse provide a hedging portfolio for the respective assets. We promote sparsity in these hedging portfolios by adding a few common factors to the hedge regression. Crucially, the factors are the same for all assets, hence we obtain a sparse inverse.

The method we propose shares common ground with several strands of previous research. Among the works closely related to ours is the Shrinkage estimator of Ledoit and Wolf (2003) and Ledoit and Wolf (2004). They propose a convex combination of the sample covariance matrix and a well-conditioned target. They guarantee that their final estimate is invertible and well-conditioned by estimating a sufficiently large weight for the target matrix in the combination. The weight they use is based on an estimator first derived under the restriction that the same shrinkage is applied to all entries, Ledoit and Wolf (2012) later extend this to allow for individual weights. Shrinkage can also be implemented under a strictly economic rationale. Jagannathan and Ma (2003) show how short selling constraints imply certain type of shrinkage on the covariance matrix.

An attractive target for the shrinkage estimator is the covariance matrix implied by a factor model of financial returns. These representations may be economic, or financial, models such as the CAPM (Sharpe, 1964), Fama-French models (Fama and French, 1993, 2015), or macro factors as in Chen et al. (1986). Alternatively, factors can stem from a statistical perspective such as those of Ross (1976), Chamberlain and Rothschild (1983), or Kelly et al. (2018b). Fan et al. (2008) explore the the asymptotic properties of covariance matrices based on factor models, when both the number of assets and the number of observed factors increase as a function of the sample size. Fan et al. (2013) further consider nonobserved factors for the purpose of portfolio optimization, proposing the "principal 
orthogonal complement thresholding' method, POET. This method use thresholding rules to set elements of the residual covariance matrix equal to zero. Thus, enabling some deviations from the strict factor structure. Carefully estimating the threshold ultimately ensures that their estimate is invertible.

Both the POET and Shrinkage procedures are well suited to accommodate commonalities in returns. Both estimators provide an estimate of the covariance matrix, which for portfolio allocation then is inverted. Goto and $\mathrm{Xu}$ (2015) propose an alternative way, which targets the inverse covariance matrix directly. They achieve this by using the penalized likelihood estimator of Yuan and Lin (2007), optimized using the graphical lasso (Friedman et al., 2008). The graphical lasso regularizes the estimate of the inverse covariance matrix by shrinking all entries and setting small elements equal to zero. Thus, from a financial perspective it performs hedging portfolio selection. However, with common factors among returns the hedging selection becomes more complicated. Assets that strongly co-vary based on common factors will appear to have a large number of important hedging relations. Put differently, the inverse covariance matrix is not necessarily closely approximated by a sparse estimate.

In all, previous methods either impose structure to improve the covariance estimate or attempt to circumvent the large- $N$ inversion. Whereas the former must still perform a large- $N$ matrix inversion, the latter requires restrictive sparsity assumptions. The contribution of this chapter is to target the inverse directly while also making use of common factors. There are several statistical methods that would make it possible to circumvent the matrix inversion. Among the viable estimators, the graphical lasso is especially well suited for portfolio optimization since it always produce estimates that are symmetric and positive semi-definite. With these properties guaranteed, it is straightforward to calibrate the regularization to specifically minimize the portfolio volatility. From a financial perspective, the graphical lasso performs optimal hedging portfolio selection. This implies that the hedges are selected to complement the given factor structure. This role is analogous to how the POET and Shrinkage estimators enables deviations from the strict factor representation. The difference is that we introduce these deviations in the inverse covariance matrix.

We evaluate the performance of the Factor-Graphical method using returns of the S\&P500 companies. We maintain a daily minimum-variance allocation based on estimates that are updated monthly or quarterly. Conditional on the preceding 90,150 or 250 days, we get six distinct cases, which we use to assess 
our performance relative to competing methods. The portfolio allocations are evaluated in the out-of-sample periods between re-estimation dates. We use the Fama-French 5-factor model as our factor structure. With daily financial returns from 2005 until 2017 we find that our method reduces risk more than competing methods such as the Shrinkage, POET and Graphical methods. The relative gains in terms of portfolio volatility using our method is between ten and fifteen percent lower than the second best performing method. Combining performance measures into an accumulated investor utility we find that our method tend to be favored by investors with high risk aversion. To assess the statistical significance of these results we implement a standard block-bootstrapping procedure to estimate the standard errors of the performance measures. We find that the additional volatility reduction generated by the Factor-Graphical method is statistically significant.

The rest of the chapter is outlined as follows. Section 3.2 introduces the financial framework and motivates our approach. Section 3.3 presents the graphical lasso along with a set of closely related estimators. In Section 3.4 we evaluate our method empirically on the S\&P500 and implement a re-sampling approach to assess the uncertainty in our performance measures. Last, Section 3.5 concludes our findings and outlines some possible direction for future research.

\subsection{Financial framework for portfolio optimiza- tion}

The method we propose relies heavily on statistical regularization. Generally regularized estimators are powerful prediction tools that reduce the impact of noise, which is a major issue with respect to financial returns. The estimators imply, however, assumptions of sparsity; many elements of the inverse are zero. Clearly the financial assumptions about returns and common factors have major implications for what statistical assumptions are suitable. This section outlines our financial framework for asset returns and informs us of where to find reliable sparsity assumptions for our estimation.

Our goal is a minimum variance portfolio for $N$ risky assets. The allocation is derived through the optimization,

$$
\min _{w} w^{\prime} \Sigma w \quad \text { subject to } \quad \iota^{\prime} w=1
$$


where $\Sigma$ denotes the $N \times N$ covariance matrix of returns, $\iota$ is a conforming vector of ones, and $w$ are portfolio weights. The asset allocation that solves this minimization is

$$
w=\frac{\Sigma^{-1} \iota}{\iota^{\prime} \Sigma^{-1} \iota} .
$$

When $N$ is large, the empirical challenge is estimating the covariance matrix (Kan and Zhou, 2007). The sample covariance matrix is insufficient due to estimation errors, which are further amplified through the matrix inversion. Our approach relies on financial factors and statistical regularization to reduce measurement error, while also circumventing the large- $N$ matrix inversion.

\subsubsection{A financial framework for the inverse covariance ma- trix}

Starting from a linear representation of asset returns, we decompose the covariance matrix and its inverse. Here the factors are assumed to be orthogonal to the idiosyncratic component, such that we can exploit the factor structure to decompose the covariance matrix of returns. Let $N$ returns be represented by a factor model of $K$ factors and uncorrelated idiosyncratic errors,

$$
y=\alpha+\boldsymbol{B} F+e,
$$

where $F$ is a $K$-vector of factors and $y$ is a $N$-vector of financial returns, we assume that $K<N$. The asset exposures are $\boldsymbol{B}$ a matrix of dimensions $N \times$ $K$. Idiosyncratic errors have mean zero and a covariance matrix $\Delta$, the factor covariance matrix is $\Psi$. Under a strict factor structure the idiosyncratic covariance matrix $\Delta$ and its inverse $\Delta^{-1}$ are both diagonal. We relax this assumption to allow for a sparse $\Delta^{-1}$ with some non-zero off-diagonal elements. Imposing the assumption on the inverse sets us apart from previous methods that assume that the idiosyncratic covariance matrix is sparse. Denoting covariances between factors and returns $\Xi$, we partition the joint covariance matrix of factors and returns $\Omega$,

$$
\Omega=\left(\begin{array}{cc}
\Psi & \Xi \\
\Xi^{\prime} & \Sigma
\end{array}\right)=\left(\begin{array}{cc}
\Psi & \boldsymbol{B} \Psi \\
\Psi \boldsymbol{B}^{\prime} & \boldsymbol{B} \Psi \boldsymbol{B}^{\prime}+\Delta
\end{array}\right) .
$$


Assuming that factors are non-traded, the minimum variance allocation requires an estimate of the marginal inverse, $\Sigma^{-1}$, the inverse covariance matrix of returns, ${ }^{3}$

$$
\Sigma^{-1}=\left(\boldsymbol{B} \Psi \boldsymbol{B}^{\prime}+\Delta\right)^{-1}=\Delta^{-1}-\Delta^{-1} \boldsymbol{B}\left(\boldsymbol{B}^{\prime} \Delta^{-1} \boldsymbol{B}+\Psi^{-1}\right)^{-1} \boldsymbol{B}^{\prime} \Delta^{-1} .
$$

The inverse covariance matrix of returns is the difference between the idiosyncratic inverse and the effect of the marginalization, summarized in the latter term. Importantly, (3.5) reveals that $\Sigma^{-1}$ is not sparse despite a sparse $\Delta^{-1}$. Assuming a statistically sparse $\Sigma^{-1}$ is therefore in conflict with the common factor structure (3.3). Our proposition is instead to approach factors and returns jointly. Inverting the covariance matrix of factors and returns reveals that the joint inverse is sparse,

$$
\Omega^{-1}=\left(\begin{array}{cc}
\Psi^{-1}+\boldsymbol{B}^{\prime} \Delta^{-1} \boldsymbol{B} & -\boldsymbol{B}^{\prime} \Delta^{-1} \\
-\Delta^{-1} \boldsymbol{B} & \Delta^{-1}
\end{array}\right) .
$$

Hence, when the number of factors is small we find that $\Omega^{-1}$ is statistically sparse. The joint inverse contains all terms needed to estimate $\Sigma^{-1}$ using (3.5). Importantly, the partition reveals that the only high-dimensional estimation challenge is the idiosyncratic inverse. This matrix is conditionally sparse, which makes it well-suited for regularized estimation. The remaining terms, $\boldsymbol{B}$ and $\Psi$, do not satisfy sparsity restriction. However, with a small number of factors the matrix $\Psi$ is low-dimensional and estimating $\boldsymbol{B}$ only requires a low-dimensional regression for each individual asset. Thus, to estimate a high-dimensional $\Sigma^{-1}$ it is only the estimate of $\Delta^{-1}$ that requires regularization. All other terms can be solved with standard statistical methods.

The approach we refer to as the Factor-Graphical method uses the structure to pursue the estimation of $\Sigma^{-1}$ through two stages. First, estimating $\boldsymbol{B}$ and $\Psi$ using standard non-regularized methods. Second, conditional on the factors estimating the high-dimensional $\Delta^{-1}$ using regularized methods. Economically these stages represent a separation of common factor exposure and hedging portfolio selection. To accommodate the large number of assets the hedging portfolios are restricted by a sparsity assumption. The assumption is motivated through the separate

\footnotetext{
${ }^{3}$ While the Fama-French factors are valid portfolios we do not consider them as viable investment options. If the factors correspond to viable investments there is no reason to omit them from the portfolio, in this case the minimum variance allocation instead requires an estimate of $\Omega^{-1}$.
} 
consideration of the common factors. Combining the estimates of our two stages using (3.5) provides an estimate of $\Sigma^{-1}$. The next section introduces a simulation exercise to illustrate that the distinction is crucial for high-dimensional estimation methods and portfolio allocation.

\subsubsection{Illustrating the Factor-Graphical approach by simula- tion}

As further motivation of our approach we perform a small simulation study where we compare our Factor-Graphical methodology to the direct marginal inverse estimation, proposed by Goto and $\mathrm{Xu}$ (2015). We simulate a set of $N=100$ assets under a factor model of $K=20$ observed factors over $T=200$ observations. $^{4}$ Idiosyncratic errors are drawn from a multivariate normal distribution with fast decreasing cross-asset correlation. Thus, the error of series $i$ is strongly correlated with series $i+1$ and other close neighbors but essentially uncorrelated with the other error series. Full details of the simulation design can be found in Appendix A. The decaying correlation pattern generates a inverse covariance matrix that conditionally on the factors is sparse. Factors are drawn from independent normal distributions with the respective loadings uniformly distributed. We calibrate the error variance such that the univariate fit of the factors is equal to 50 percent. Thus, the simulation setup is not a strict factor structure but there are strong commonalities in the covariation of the series.

Using this setup it is possible to estimate the marginal inverse either directly or through the idiosyncratic inverse and (3.5), which is the Factor-Graphical method. Estimating the marginal inverse using both approaches we plot the distribution of off-diagonal elements and compute the accuracy of the respective estimates using the Frobenius norm. Figure 3.1 display the respective distributions of absolute off-diagonal elements.

The distributions are computed over $L=1000$ simulations. The frequencies are scaled such that the counts correspond to the frequencies per simulation and the off-diagonal elements are multiplied by 1000 . The distribution of the offdiagonal elements in the estimate based on the idiosyncratic inverse is denoted by the solid line. This distribution follows the pattern of the actual simulated distribution pretty closely. The estimator that targets $\Sigma^{-1}$ directly fares much worse. The two-dashed line denotes the off-diagonal elements of this estimator and

\footnotetext{
${ }^{4}$ In our empirical application we make the dimensionality issues even more severe by allowing $N$ to far exceed $T$.
} 
Figure 3.1: Off-diagonal elements of estimates $\hat{\Sigma}^{-1}$

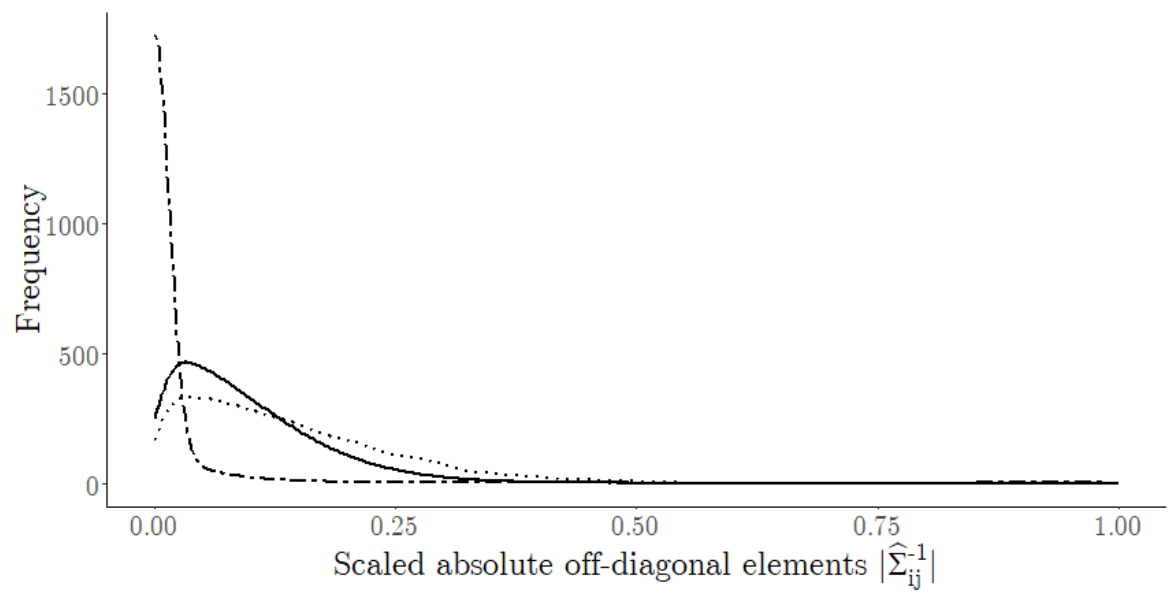

Distribution of absolute off-diagonal elements. Frequencies are averaged over $L=1000$ simulations. Factor-Graphical (solid) and the direct marginal inverse estimate(dashed) is plotted along the actual simulated distribution (dotted). Both estimates employ regularization that promotes sparsity. Off-diagonal elements are multiplied by 1000 . The simulations are performed using a fixed design.

fails to replicate the distribution of the actual data. Since this estimator regularizes $\Sigma^{-1}$ using a penalization that promotes sparsity we find that the distribution of elements deviates much from the factor structure. The sparsity of the estimate is far too extreme compared to the actual distribution of off-diagonal elements. Figure 3.1 provides a view of the bias of the respective methods. Next we turn to the uncertainty in the estimate as measured by the standard errors. Figure 3.2 shows the distribution of the Frobenius distance between the estimated inverse and the actual inverse covariance matrix over the simulations.

The estimate based on the idiosyncratic inverse and the factors provides a more precise estimate of the actual inverse. It appears that the common factors generates an inverse that the direct marginal inverse cannot recover. Specifically, the common factor structure works against the sparsity assumption of the regularized estimators. The idiosyncratic inverse, however, is sparse. Therefore we find that the accuracy of the Factor-Graphical approach is always more accurate than directly estimating the marginal inverse in all of the $L$ simulations. 
Figure 3.2: Fit of the estimate $\widehat{\Sigma}^{-1}$ to actual $\Sigma^{-1}$

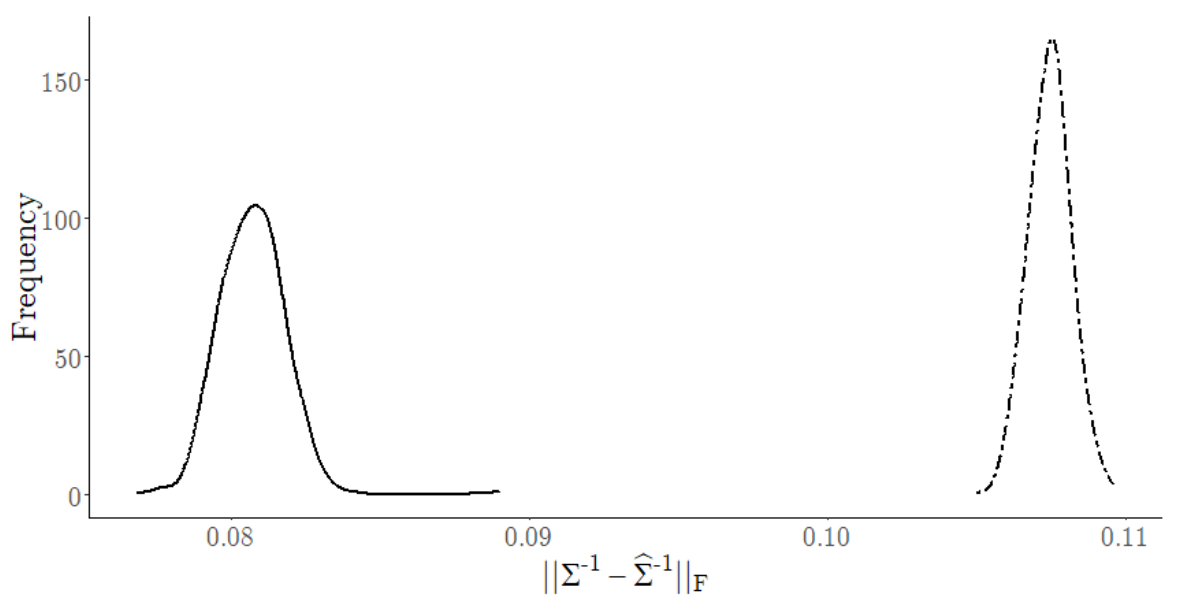

Frobenius norm of difference between the simulated marginal inverse $\left(\Sigma^{-1}\right)$ and estimates $\left(\widehat{\Sigma}^{-1}\right)$. Distribution of norm over $L=1000$ simulations using the Factor-Graphical(solid) estimate and using the direct marginal inverse estimate(dashed). Both estimates employ regularization that promotes sparsity.

In reality the factor model is unknown and to some degree misspecified, which is partly reflected in our simulation set up. ${ }^{5}$ Clearly, if all factors of a model are redundant the Factor-Graphical approach is reduced to the method of targeting $\Sigma^{-1}$ directly. However, under non-redundant factor structures the partition improves the risk reduction, increasingly so when factors fit the returns more closely.

\subsection{Estimating the idiosyncratic inverse}

The economic framework we have set up can be used to estimate a minimum variance portfolio but relies on the inverse of a large- $N$ idiosyncratic matrix. We estimate this inverse directly, hence avoiding the explicit large- $N$ inversion. To make this point explicit in our notation we refer to the idiosyncratic inverse as $\Theta$.

We estimate the idiosyncratic inverse, conditional on the Fama-French factors, of the assets from (3.6). To estimate the inverse we use the negative log-likelihood, augmented by a penalization on the sum of the absolute elements. The regulariza-

\footnotetext{
${ }^{5}$ The issue of factor model misspecification is interesting with respect to what type of regularization is optimal. A full treatment of that issue is beyond the scope of this chapter but Appendix 3.A provides a motivation for the choice we pursue here.
} 
tion is here imposed on the first norm of the off-diagonal elements. This estimator was proposed by Yuan and Lin (2007), let $\Theta \succeq 0$ denote the set of positive semidefinite matrices,

$$
\widehat{\Theta}=\operatorname{argmin}_{\Theta \succeq 0}\left(\operatorname{tr}(\widehat{S} \Theta)-\log (\operatorname{det}(\Theta))+\Lambda\|\Theta\|_{1}\right),
$$

where $\widehat{S}=T^{-1} \widehat{E}^{\prime} \widehat{E}$, the covariance matrix of the residuals from (3.3) conditional on a set of factors. The estimate $\widehat{\Theta}$ along with the asset exposures $\widehat{\boldsymbol{B}}$ and $\widehat{\Psi}^{-1}$ gives us an estimate for the marginal inverse $\widehat{\Sigma}^{-1}$ through (3.5). The factor covariance matrix, $\Psi$, is of low dimension and estimated by the sample covariance matrix does not cause any inversion problems.

In this section we present one of the most popular methods for solving the optimization, the Glasso algorithm (Friedman et al., 2008). We also present an alternative method not based on the likelihood (3.7) but instead forming the estimate through $N$ nodewise regressions. This is known as the neighborhood regularization method (Meinshausen and Bühlmann, 2006). This method does not impose properties such as symmetry or positive semi-definiteness on the estimate. However, the assumptions of the two alternatives differ.

In the statistical literature Fan, Liu, and Wang (2018) study the theoretical properties of a related approach that also impose conditional sparsity in the inverse covariance matrix. The method they study does not rely on the graphical lasso to promote sparsity, instead they make use of the 'constrained $\ell_{1}$-minimization for inverse matrix estimation', CLIME, estimator (Cai, Liu, and Luo, 2011). This estimator achieves some statistical properties, such as consistent model selection, under less restrictive assumption than the graphical lasso. However, this estimator does not impose positive semi-definiteness on the estimate. As a consequence it is less well tailored for our purpose.

\subsubsection{The graphical lasso}

For the purposes of financial portfolio allocation for large $N$ we propose solving the likelihood optimization of (3.7) by using the Glasso algorithm of Friedman et al. (2008). To introduce their algorithm define a partition of the inverted target covariance matrix, in this case $\Theta$, and for later notational convenience the inverse 


$$
\begin{aligned}
& C=\Theta^{-1}, \\
& \quad \Theta=\left(\begin{array}{ll}
\Theta_{11} & \theta_{12} \\
\theta_{21} & \theta_{22}
\end{array}\right), \quad C=\left(\begin{array}{ll}
C_{11} & c_{12} \\
c_{21} & c_{22}
\end{array}\right),
\end{aligned}
$$

where $\Theta_{11}$ has dimensions $(N-1) \times(N-1), \theta_{12}$ and $\theta_{21}$ are both of $(N-1) \times 1$ and $\theta_{22}$ is scalar. Analogously define the same partition of the covariance matrix and the elements-wise sign matrix, $\Gamma=\operatorname{sign}(\Theta)$,

$$
S=\left(\begin{array}{ll}
S_{11} & s_{12} \\
s_{21} & s_{22}
\end{array}\right), \quad \Gamma=\left(\begin{array}{ll}
\Gamma_{11} & \gamma_{12} \\
\gamma_{21} & \gamma_{22}
\end{array}\right) .
$$

The algorithm uses these matrix partitions to optimize penalized log-likelihood (3.7), $Q=-\log (\operatorname{det}(\Theta))+\operatorname{tr}(\widehat{S} \Theta)+\Lambda\|\Theta\|_{1}$. Friedman et al. (2008) start by considering the first order conditions,

$$
-\Theta^{-1}+S+\Lambda \Gamma=0 .
$$

In order to separate these conditions along the lines of the partitions of (3.9) it is convenient to express the partition of $C$ as a function of the elements of $\Theta$. Hence write this partition as,

$$
C=\left(\begin{array}{cc}
\left(\Theta_{11}-\theta_{12} \theta_{22}^{-1} \theta_{21}\right)^{-1} & -C_{11} \theta_{12} \theta_{22}^{-1} \\
\left(-C_{11} \theta_{12} \theta_{22}^{-1}\right)^{\prime} & \left(\theta_{22}-\theta_{21} \Theta_{11}^{-1} \theta_{12}\right)^{-1}
\end{array}\right) .
$$

As we separate the normal equations (3.10) into their respective blocks we immediately write the condition on the $N$ :th row/column under the partition (3.11),

$$
C_{11} \theta_{12} \theta_{22}^{-1}+s_{12}+\lambda \gamma_{12}=0
$$

Here it is convenient to define $\beta=\theta_{12} \theta_{22}^{-1}$. With the normal equations rewritten in this manner Friedman et al. (2008) point out that these conditions are the optimality conditions of the minimization problem,

$$
\hat{\beta}=\operatorname{argmin}\left(\frac{1}{2} \beta^{\prime} C_{11} \beta+\beta^{\prime} s_{12}+\lambda|\beta|\right) .
$$


Initializing the algorithm under some initial $C$ they solve this optimization for an estimate $\hat{\beta}$ and update $c_{12}$ using the partition in (3.11). Iteratively changing the target column the algorithm updates the estimate column-by-column until the adjustments fall below a given threshold. Upon convergence they ultimately update the estimate of the inverse, $\hat{\theta}_{22}=\left(c_{22}-c_{12}^{\prime} \hat{\beta}\right)^{-1}$ and $\hat{\theta}_{12}=-\hat{\beta} \hat{\theta}_{11}$. There

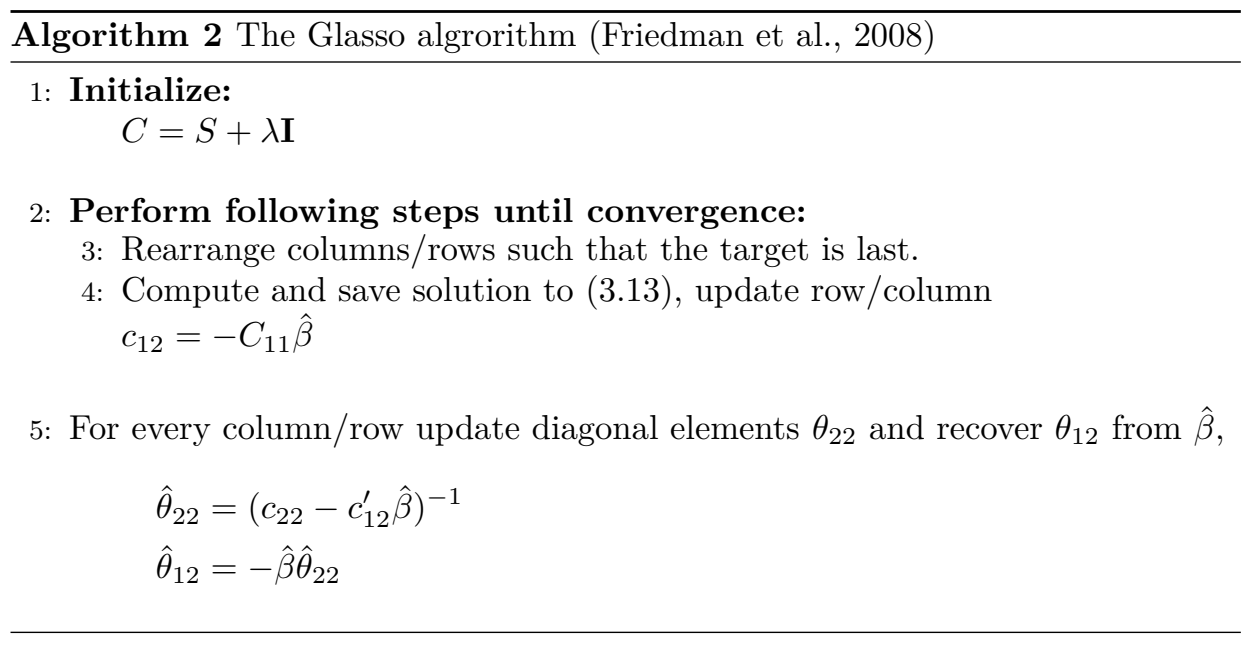

are some known drawbacks to this algorithm. Mazumder and Hastie (2012) show that unless the algorithm converges there is no guarantee that the estimate of $\Theta$ will be positive semi-definite. This approach is ill-suited to situations where the we seek a computationally fast approximation of a high-dimensional inverse. This is the case where the dimensions of the data are so immense that there is no practical way of reaching convergence. It is from this observation they develop an algorithm that constructs a positive semi-definite inverse at every iteration. However, for our purposes the computational cost is not high enough to warrant an implementation of their algorithm. Therefore, we will continue under the framework outlined above.

We initialize the algorithm by setting the matrix $S$ equal to the sample covariance matrix of the residuals of the Fama-French factor model. Estimating $\Theta$ we supply a strictly positive penalization parameter grid, $\lambda_{n} \cdot{ }^{6}$ Under each value of the penalization grid we estimate an inverse. To find the optimal level of penalization we rely on a 5 -fold cross-validation scheme. Through cross-validation

\footnotetext{
${ }^{6}$ We follow common practice and select the highest level of regularization equal to $\lambda_{\max }=$ $\max (\max (S-\boldsymbol{I}),-\min (S-\boldsymbol{I}))$ and the smallest level $\lambda_{\min }=0.1 \lambda_{\max }$ The grid between these boundaries is composed of 50 values linearly decreasing in log scale.
} 
we are able to directly search for the estimate that provides the lowest portfolio risk. Hence, we implement the cross-validation to find the allocation that implies the lowest portfolio volatility across the 5 -folds. Randomly assigning observations into one of the five folds such that the folds remain largely equal in size, the procedure uses four fold as the estimation window to estimate the inverse and evaluates the estimates against the observations in the omitted fifth fold. Within each estimation window we estimate the inverse over a grid of regularization. We choose the level of regularization, which produces the lowest average portfolio volatility across all five validation folds. Yuan and Lin (2007) first study the properties of the estimator when the number of variables is fixed and the sample size increases. Rothman et al. (2008) found that with normally distributed i.i.d. variables under a high-dimensional framework the graphical lasso is consistent in the Frobenius norm assuming that the minimum and maximum eigenvalues of the covariance matrix are bounded. They further show that the estimator is consistent at the rate $O_{p}(\sqrt{(N+s) \log (N) / T})$, where $s$ denotes the number of non-zero elements of the inverse covariance matrix. Ravikumar et al. (2011) further find that these statistical properties hold for a large class of distributions beyond the multivariate Gaussian. They show that under irrepresentability conditions the estimator is consistent and they derive the decrease in the maximum norm of the element-wise error. ${ }^{78}$ Under the same assumptions they also prove model selection consistency, the estimator uncovers the true cross-variable relations with the correct sign. In all, for the context at hand these papers further underscore the importance of accounting for the common factor structure among returns.

With the estimator calibrated, either through cross-validation or the information criteria, we estimate $\widehat{\Theta}$ over the whole estimation window. From a financial point of view this estimate describes the cross-asset hedging relations that complements the factor models. Using (3.5) we combine this estimate with the common factors to estimate the marginal inverse, $\widehat{\Sigma}^{-1}$. Using this estimate we form a minimum variance allocation along the lines of (3.2). We will hence refer to the portfolio based on this allocation as the Factor-Graphical portfolio.

Caner et al. (2017) form minimum variance portfolios using an estimator that is related to the graphical lasso. Their method broadly known as a neighborhood selection algorithm and may be written as a special case of the graphical lasso.

\footnotetext{
${ }^{7}$ Similar, but not equivalent to irrepresentability conditions associated with the lasso.

${ }^{8}$ From a graphical perspective the condition is that non-edges in the graph may not be highly correlated with the true edge set. In the phrasing of our application, spurious hedging relations may not be highly correlated with relevant hedging relations.
} 
Neighborhood selection estimators rely on different statistical assumptions and it is not clear a priori which is more restrictive with respect to portfolio allocation.

\subsubsection{Inverse estimation through Neighborhood Selection}

In the statistical literature there are several approaches to graphical model selection. An alternative to the graphical lasso is neighborhood selection (Meinshausen and Bühlmann, 2006). These methods estimate the inverse covariance matrix through a large number of regression equations. ${ }^{9}$ Compared to the graphical lasso the neighborhood selection methods are computationally faster and offers some additional flexibility in how we regularize the estimate. Drawback are however that these methods do not guarantee that the estimate holds properties such as symmetry and positive semi-definiteness.

Let $e_{i}$ denote a column of the residual covariance matrix $T^{-1} \widehat{E}^{\prime} \widehat{E}$. The neighborhood selection method is an estimator based on a set of $N$ node-wise regressions, $e_{i}=e_{-i} \phi_{-i}+\varepsilon_{i}$, where $\phi_{-i}$ is a parameter vector of $(N-1)$ elements. Since the number of parameters to estimate far exceeds the historical return they implement a regularized estimator,

$$
\hat{\phi}_{-i}^{(\alpha)}=\operatorname{argmin}\left(\left\|e_{i}-e_{-i} \phi_{-i}\right\|+\tilde{\Lambda}_{i}\left\|\phi_{-i}\right\|_{\alpha}\right)
$$

where $\hat{\phi}_{-i}^{(\alpha)}=\left\{\hat{\phi}_{i, p} ; p=1 \ldots N, p \neq i\right\}$. For our purposes we implement this approach under two alternative regularizations, (1) the least absolute shrinkage selection operator estimator (lasso) and (2) the ridge estimator. The ridge estimator regularizes the estimate through shrinkage without promoting sparsity in the inverse while the lasso also promotes sparsity. Setting the parameter $\alpha$ equal to one puts the penalization on the absolute parameter vector, this corresponds to a lasso estimator. Alternatively we put the penalization onto the $\ell_{2}$ norm of the parameter vector, which provides the ridge estimator. This approach is a straightforward implementation of hedging portfolio estimation as discussed by Stevens (1998). The lasso will promote portfolios of few assets while the ridge estimator will attenuate the portfolio weights but keep hedging portfolios large.

Using either estimator we use the point estimates of (3.14) to construct the inverse covariance matrix. The regression of the $i$ :th asset return onto all other

\footnotetext{
${ }^{9}$ This type of neighborhood selection approach is explored by Caner et al. (2017) but without accounting for mutual risk.
} 
returns identifies the $i$ :th row of the inverse covariance matrix, $C$.

$$
\widehat{\Theta}=\left(\begin{array}{cccc}
\hat{\theta}_{1,1}^{2} & \hat{\theta}_{1,2} & \ldots & \hat{\theta}_{1, p} \\
\hat{\theta}_{2,1} & \hat{\theta}_{2,2}^{2} & \ldots & \hat{\theta}_{2, p} \\
\vdots & \vdots & \ddots & \vdots \\
\hat{\theta}_{p, 1} & \hat{\theta}_{p, 2} & \ldots & \hat{\theta}_{p, p}^{2}
\end{array}\right)
$$

Let $\hat{\varepsilon}_{i}$ denote the residual of the $i$ :th regression under the parameter vector $\hat{\phi}_{-i}^{(\alpha)}$. Using these residuals we find the elements of the inverted covariance matrix as $\hat{\theta}_{i, i}^{2}=\operatorname{Var}\left(\hat{\varepsilon}_{i}\right)^{-1}$ and $\hat{\theta}_{i, j}=-\hat{\phi}_{i, j}^{(\alpha)} / \operatorname{Var}\left(\hat{\varepsilon}_{i}\right)$. Here the non-zero entries of the $i$ :th row are said to be in the neighborhood of the $i$ :th asset. By the sparsity imposed by the node-wise lasso estimates $\hat{\phi}_{-i}^{(\alpha)}$ we also impose sparsity on the estimated inverse. Sparsity is here defined on the cardinality of the parameter vectors $S_{j}:=\left\{j ; \phi_{-i} \neq 0\right\}$. In this context implies that each return is associated with a small neighborhood of assets, where small is defined in relation to the time dimension $T$. We calibrate the regularization parameter through $\mathrm{K}$-fold crossvalidation. Specifically we use a five fold cross-validation of the nodewise predictive performance to calibrate the regularization.

Meinshausen and Bühlmann (2006) also provide the theoretical properties of this approach when applied to the lasso regularization. They find that under normally distributed i.i.d. variables the nodewise lasso estimator is consistent for model selection under beta-min and irrepresentability conditions (Zhao and $\mathrm{Yu}, 2006) .{ }^{10}$ The irrepresentability conditions established by Zhao and $\mathrm{Yu}$ (2006) for the univariate regression setting restrict the covariation between relevant and irrelevant predictors. For the type of neighborhood selection we consider these conditions applies for all $N$ nodewise regressions. Hence, the restriction applies to $N$ covariance matrices of dimensions $(N-1) \times(N-1)$. Just like the Graphical estimator, Meinshausen and Bühlmann (2006) find that the initial Gaussian assumption does not appear to be crucial but can be replaced by more general conditions on the tail behavior of the variables. Wainwright (2009) for example finds that the lasso remains consistent, with some conditions on the sample growth rate, under sub-Gaussian error terms.

The neighborhood selection estimation approach, under all regularizations, suffer from drawbacks. None of these estimators impose expected properties such as

\footnotetext{
${ }^{10}$ The beta-min condition implies that the linear relation between the weakest relevant predictor is bound from below.
} 
symmetry and positive semi-definiteness on the estimate. To ensure that the resulting empirical concentration matrix is positive semidefinite we need to rely on eigenvalue cleaning procedures. Hautsch et al. (2012) propose a cleaning procedure based on separating the noise-driven eigenvalues from those arising due to signal in the financial markets. The latter eigenvalues remain unaffected while noise-driven values are adjusted away from zero. Let $v_{i}$ denote an eigenvalue and $V_{i}$ the corresponding eigenvector. We apply a procedure that adjusts all negative eigenvalues to equal to the smallest positive value, ${ }^{11}$

$$
\hat{v}_{i}= \begin{cases}v_{i} & \text { if } v_{i}>0 \\ \bar{v}, & \text { otherwise }\end{cases}
$$

where $\bar{v}=\min \left(v_{i} \mid\left(v_{i}>0\right)\right)$. Using the vector of cleaned eigenvalues, $\hat{v}$, along with corresponding eigenvectors we can project a semidefinite inverse, $\widetilde{\Theta}=V \operatorname{diag}(\hat{v}) V^{-1}$. Using this estimate we may again proceed with the portfolio allocation under the minimum variance allocation (3.2). In our empirical evaluation we refer to the portfolios allocated under these estimators as Nodewise $\ell_{1}$ and Nodewise $\ell_{2}$.

\subsection{Minimum variance allocation of the S\&P500}

In this section we evaluate the performance of the Factor-Graphical method of portfolio allocation using S\&P500 companies. We first introduce our data and the financial performance measures we use to assess performance. The main results that follow compares our financial performance against the main alternative methods: Shrinkage, POET, Neighborhood selection and $1 / N$. Full details of how we implement the Shrinkage and POET approaches are presented in Appendix C. The $1 / N$ rule allocates the total wealth equally over all assets. While seemingly naive this allocation rule has often proved difficult to outperform empirically (DeMiguel et al., 2007). Following our main performance comparison we analyze whether our performance stems disproportionately from the factors or the hedging portfolios by contrasting our performance against two restricted versions of the method. The first is a strict factor model. The second applies a graphical lasso directly to the returns without conditioning on factors. Finally we assess the statistical uncertainty of the estimated performance gains through bootstrapping.

\footnotetext{
${ }^{11}$ Since there is no need to invert this matrix we do not need to worry about possible numerical issues associated with small eigenvalues.
} 


\subsubsection{Data and performance measures}

Daily asset returns are collected from the CRSP database spanning the years 2010 until 2018. Since the companies comprising the S\&P500 vary over time we include all companies that at some point were included during the period 2005-2017. This provides a set of almost 800 assets. The portfolios are allocated using estimates that are updated either at a monthly or quarterly frequency. At the point of re-estimation the respective procedures are provided an estimation window of one among three sizes, $\tilde{t}=\{90,150,250\}$, of historical daily returns. The estimated allocation is maintained under daily re-balancing until the next re-estimation. Total number of returns are denoted $T$. Basic descriptive statistics and individual asset performance measures are displayed in Appendix 3.B. An asset that is missing as some point in the estimation window is given the weight zero at the corresponding re-estimation.

We compare the performance of all the estimation methods by the out-ofsample volatility, Sharpe ratio, turnover and investor utility under a quadratic utility function including transaction costs. The monthly updates is based on estimates that are updated every 30 days. This gives $M=(T-\tilde{t}) / 30$ portfolio allocations, indexed $m=1 \ldots M$, each used over the next 30 days. The portfolio weights $\hat{\mathbf{w}}_{m}^{P}$ are estimated using observations preceding the month $m$. We compute the realized average daily return and portfolio volatility for each method $P$,

$$
\begin{aligned}
& \hat{\mu}_{P}=(T-\tilde{t})^{-1} \sum_{m=1}^{M} \sum_{d=1}^{30} \hat{\mathbf{w}}_{m}^{P} r_{m, d}, \\
& \hat{\sigma}_{P}=\sqrt{(T-\tilde{t}-1)^{-1} \sum_{m=1}^{M} \sum_{d=1}^{30}\left(\hat{\mathbf{w}}_{m}^{P} r_{m, d}-\hat{\mu}_{P}\right)^{2}},
\end{aligned}
$$

where $\mathbf{w}_{m}^{P}$ are weights and $\mathbf{r}_{m, d}$ is a vector of returns at day $d$. With the realized returns and the portfolio volatility we compute the Sharpe ratio $S R_{P}=\hat{\mu}_{P} / \hat{\sigma}_{P}$. Due to transaction costs on financial markets an important performance metric is how much trade is required to maintain a portfolio allocation. Let the turnover of forming the portfolio at day $d$ of month $m$ be denoted $\delta_{m, d}^{P}$. The average turnover 
is,

$$
\hat{\delta}_{P}=(T-\tilde{t})^{-1} \sum_{m=1}^{M} \sum_{d=1}^{30} \sum_{i=1}^{N}\left|\widehat{w}_{i, m}^{P}-\widehat{w}_{i, m}^{*} \frac{1+r_{i, m, d+1}}{1+\widehat{\mathbf{w}}_{m}^{P} \mathbf{r}_{m, d+1}}\right|
$$

The vector $\mathbf{r}_{m, d+1}$ denotes the returns on $d+1$ of month $m$ and the weights $\widehat{w}_{i, m}^{*}$ are defined,

$$
\widehat{w}_{i, m}^{*}= \begin{cases}\widehat{w}_{i, m-1}^{P}, & \text { if } d=1 \\ \widehat{w}_{i, m}^{P}, & \text { if } d>1\end{cases}
$$

To provide a measure that combines the turnover, portfolio return and the volatility, we use a utility based approach similar to Fleming et al. (2001, 2003). Assuming a quadratic utility function for an investor with risk aversion $\kappa$, we get the realized utility

$$
U_{m, d}^{P}=\left(1+\mathbf{w}_{m}^{P} \mathbf{r}_{m, d}\right)\left(1-c \delta_{m, d}^{P}\right)-\Upsilon\left(\left(1+\mathbf{w}_{m}^{P} \mathbf{r}_{m, d}\right)\left(1-c \delta_{m, d}^{P}\right)\right)^{2} .
$$

Here $\Upsilon=\kappa /(2(1+\kappa))$ and $\kappa$ denotes the relative risk aversion. The parameter $c$ denotes transaction costs, which are imposed proportionally to the turnover. We follow Garlappi et al. (2007) and set the costs equal to 50 basis points. To compare the different methods we compute how large a constant daily fee must be for investors to become indifferent between two methods. Specifically, we find the fee, $\chi$, such that the utility gain under the Factor-Graphical method equals that of the competing method, $\sum_{m=1}^{M} \sum_{d=1}^{30} U_{m, d}^{F G}(\chi)=\sum_{m=1}^{M} \sum_{d=1}^{30} U_{m, d}^{P}$ where

$$
\begin{aligned}
U_{m, d}^{F G}(\chi)=\left(\left(1+\mathbf{w}_{m}^{F G} \mathbf{r}_{m, d}\right)\right. & \left.\left(1-c \delta_{m, d}^{F G}\right)-\chi\right) \\
& -\Upsilon\left(\left(1+\mathbf{w}_{m}^{F G} \mathbf{r}_{m, d}\right)\left(1-c \delta_{m, d}^{F G}\right)-\chi\right)^{2}
\end{aligned}
$$

Finding that $\chi$ is negative implies that the realized utility of the Factor-Graphical method is lower than the competing method. We compute the performance measure under the quarterly updated estimates analogously.

\subsubsection{Factor-Graphical portfolio performance}

This subsection presents our main results. The Factor-Graphical portfolio performance is compared to the principal methodologies of the literature. Additionally 
we consider the neighborhood selection estimators of the idiosyncratic inverse. ${ }^{12}$ We summarize the financial performance of the respective portfolio allocation in Table 3.1. The first panel displays the outcomes while the estimates are updated every 30 days, the second panel show the results of re-estimating every 90 days.

Table 3.1: Financial Performance 2010-03-31 until 2016-12-28.

\begin{tabular}{lccc|rccrrrr}
\hline \hline & \multicolumn{3}{c}{$\tilde{t}=90$} & \multicolumn{3}{c}{$\tilde{t}=150$} & \multicolumn{4}{c}{$\tilde{t}=250$} \\
\multicolumn{1}{c}{ Monthly } & $\hat{\sigma}$ & $\hat{\mu} / \hat{\sigma}$ & $\hat{\delta}$ & \multicolumn{1}{c}{$\hat{\sigma}$} & $\hat{\mu} / \hat{\sigma}$ & $\hat{\delta}$ & $\hat{\sigma}$ & $\hat{\mu} / \hat{\sigma}$ & \multicolumn{1}{c}{$\hat{\delta}$} \\
\hline Factor-Graphical & 6.09 & 1.22 & 6.53 & 6.57 & 1.51 & 8.42 & 7.65 & 1.43 & 10.50 \\
Nodewise $\ell_{1}$ & 6.67 & 0.93 & 6.91 & 7.33 & 1.30 & 7.16 & 8.47 & 1.57 & 6.67 \\
Nodewise $\ell_{2}$ & 6.52 & 1.09 & 6.67 & 7.38 & 1.26 & 7.21 & 8.43 & 1.43 & 6.73 \\
Shrinkage & 7.09 & 1.12 & 5.67 & 8.19 & 1.37 & 7.37 & 9.09 & 1.23 & 9.54 \\
POET & 8.65 & 0.68 & 4.56 & 10.18 & 0.81 & 5.34 & 11.83 & 0.95 & 6.20 \\
$\quad$ & & & & & & & & & \\
$\quad$ Quarterly & & & & & & & & & \\
Factor-Graphical & 6.70 & 0.97 & 6.76 & 6.95 & 1.23 & 8.18 & 7.85 & 1.27 & 10.28 \\
Nodewise $\ell_{1}$ & 7.15 & 0.97 & 6.81 & 7.73 & 1.21 & 7.03 & 8.72 & 1.37 & 6.44 \\
Nodewise $\ell_{2}$ & 7.18 & 1.04 & 7.04 & 7.63 & 1.23 & 6.79 & 8.73 & 1.19 & 6.43 \\
Shrinkage & 7.68 & 0.77 & 5.78 & 8.35 & 1.04 & 7.10 & 9.07 & 1.02 & 9.22 \\
POET & 8.91 & 0.64 & 4.67 & 10.06 & 0.69 & 5.22 & 11.70 & 0.82 & 6.21 \\
\end{tabular}

The volatilities $\hat{\sigma}$, turnover $\hat{\delta}$ and Sharpe ratios $\hat{\mu} / \hat{\sigma}$ are all annualized through a $\sqrt{252}$ or 252 scaling. The portfolio volatilities are scaled by 100 .

We find that the Factor-Graphical method consistently produces the lowest portfolio risk. Under the smallest estimation window, using monthly re-estimation, we find that our method produce an annualized volatility of 6.09 percent. The second best alternatives are the two Nodewise estimators, which also lowers volatility more than the covariance estimators. The best performing covariance estimator is the Shrinkage estimator, but with the much higher volatility of 7.09. As we let the estimation window grow to 250 observations the Factor-Graphical volatility increases to 7.64 percent. $^{13}$

The portfolios allocated using the covariance estimators, Shrinkage and POET, do not reduce the risk to the same extent as the Factor-Graphical nor the Nodewise estimators does. We find that the Shrinkage portfolio produce volatility varying

\footnotetext{
${ }^{12}$ The neighborhood selection estimators are not common in the literature but they are explicitly designed to tackle the challenge of high-dimensional covariance inverse estimation. One paper that explores these methods of portfolio allocation is Caner et al. (2017).

${ }^{13}$ The decrease in performance at larger sample windows is the result of an additional trade-off. Parameter instability and dynamics in the true covariance matrix along with the factor structure renders older historical returns less informative than recent news. The correct sample window size considers both the rate of decay in the informativeness and the rate of convergence in the estimator. Hence, the optimal number of observation to include is a big questions which goes beyond the scope of this chapter.
} 
between 7.09 and 9.09 percent, which is consistently lower than the volatility achieved by the POET. Across all estimation windows and re-estimation frequencies we find that the POET allocation delivers the highest portfolio volatility. With the smallest estimation window and monthly estimates the volatility of these portfolios is 8.65, which is more than 40 percent higher than our Factor-Graphical portfolios. Turning to the nodewise estimators we find small differences in performance between the lasso and ridge regularizations.

As a secondary consideration to the portfolio volatility we compute the Sharpe ratios. When we re-estimate the inverse covariance matrix monthly we find that the Factor-Graphical method produces the highest Sharpe ratio in the two smaller estimation windows. With 250 observations there is largely no difference in Sharpe ratios between the two nodewise allocations and the Factor-Graphical method. The Shrinkage portfolio largely attains similar levels of Sharpe ratios as do the nodewise allocations. As we decrease the estimation frequency to quarterly optimization, similar patterns emerge. The graphical method along with the nodewise estimators generate portfolios with higher Sharpe ratios than the Shrinkage and POET estimators.

The Factor-Graphical method generally performs better than both the other inverse matrix estimators and the main covariance estimators of the literature. The increased performance comes at the cost of higher portfolio turnover. For all estimation windows size and re-estimation frequencies the Factor-Graphical methods is among the portfolios with the highest turnover. The lowest turnover is associated with the POET estimator, which also saw the lowest risk reduction. The Shrinkage methodology, which saw lower risk reduction compared to the nodewise allocations generates much higher turnover when the sample window is large. On the other hand the Shrinkage turnover is very low in the smallest sample window of 90 days. Estimating the inverse directly under a column-by-column approach generally appears to produce much more stable portfolios as measured by the turnover.

To see if the variance reduction is large enough to compensate the increased trading costs we assess the investor utility. To quantify how large the utility gain or loss of the Factor-Graphical method we compute the constant daily fee, denoted $\chi$, necessary to make investors indifferent between the Factor-Graphical and a competing method. 
Table 3.2: Estimated equalization fee $\left(\chi_{\kappa}\right)$ on Factor-Graphical portfolio

\begin{tabular}{lrrrrrrrrr}
\hline \hline & \multicolumn{3}{c}{$\tilde{t}=90$} & \multicolumn{3}{c}{$\tilde{t}=150$} & \multicolumn{4}{c}{$\tilde{t}=250$} \\
Monthly & $\chi_{1}$ & \multicolumn{1}{c}{$\chi_{5}$} & \multicolumn{1}{c}{$\chi_{10}$} & \multicolumn{1}{c}{$\chi_{1}$} & \multicolumn{1}{c}{$\chi_{5}$} & $\chi_{10}$ & $\chi_{1}$ & $\chi_{5}$ & $\chi_{10}$ \\
\hline NW $\ell_{1}$ & 1.43 & 1.58 & 1.76 & -0.15 & 0.06 & 0.33 & -4.30 & -4.03 & -3.70 \\
NW $\ell_{2}$ & 0.44 & 0.55 & 0.69 & 0.10 & 0.33 & 0.62 & -3.00 & -2.74 & -2.43 \\
Shrinkage & -0.92 & -0.66 & -0.32 & -1.70 & -1.22 & -0.61 & -0.64 & -0.15 & 0.46 \\
POET & 0.77 & 1.53 & 2.49 & 0.45 & 1.67 & 3.21 & -2.07 & -0.42 & 1.65 \\
Quarterly & & & & & & & & & \\
\hline NW $\ell_{1}$ & -0.34 & -0.22 & -0.05 & -1.34 & -1.10 & -0.81 & -3.92 & -3.63 & -3.27 \\
NW $\ell_{2}$ & -0.79 & -0.66 & -0.49 & -1.46 & -1.26 & -1.01 & -2.34 & -2.05 & -1.68 \\
Shrinkage & 0.17 & 0.46 & 0.81 & -0.55 & -0.12 & 0.42 & 0.32 & 0.74 & 1.26 \\
POET & -0.09 & 0.61 & 1.48 & 0.44 & 1.51 & 2.85 & -1.31 & 0.22 & 2.13 \\
\hline
\end{tabular}

Nodewise $\ell_{1}$ and $\ell_{2}$ procedures are abbreviated NW. The estimated constant daily fee sufficient to equate the Factor-Graphical portfolio with the competing methods. Here $\chi_{\kappa=1}$ denotes the estimated fee when investors have low risk aversion. A negative value implies that the equalizing fee is imposed on the competing method, while a positive entries imply that the fee is put on the Factor-Graphical portfolio. As such, negative values imply investor utility under the competing method exceeds that of the Factor-Graphical portfolio.

Table 3.2 reports the annualized estimated fees. Positive fees implies that the Factor-Graphical portfolio holds the higher utility while a negative implies that the competing method is higher.

First we contrast the utility gains for high risk aversion investors. Under high risk aversion and monthly re-estimation our Factor-Graphical method tend to outperform the POET and Shrinkage estimators. Of these two the Shrinkage portfolio produce higher utility than the Factor-Graphical method in two cases, which are the cases of small sample windows and monthly re-estimation.

An interesting result emerges in the comparison between the Factor-Graphical and the nodewise estimators. Re-estimating the allocations monthly we find that Factor-Graphical portfolio is preferred by high risk aversion investors, except when the sample window is very large. In the sample window of 90 days the high risk aversion investors can accommodate an annualized daily fee of 1.76 percent before it is indifferent between the Nodewise $\ell_{1}$ allocation and the Factor-Graphical portfolio. This changes when the sample is large and when we update the estimates quarterly. In these cases the Factor-Graphical method never reach the same investor utility as any of the nodewise estimators.

Turning to the investor utility under low risk aversion we find that the results are very mixed. In no single case is the Factor-Graphical portfolio the preferred over the two covariance estimators. This is somewhat expected since the low risk aversion investors puts a smaller premium on volatility reduction. In the case of 
monthly re-estimation and a sample window of 150 observations we find that the Shrinkage portfolio can maintain a fee of 1.70 percent before the investors are indifferent between this allocation and the Factor-Graphical. In comparison to the nodewise allocations we also find mixed results for our method. Re-estimating the allocations monthly the investors prefer the graphical portfolio under small samples, but this changes as we increase the window length. Estimating the allocations quarterly the Factor-Graphical method never reach the same level as the nodewise allocations.

The last of our main results concern a recurring question for statistically driven methods of portfolio allocation; does the method outperform an equal allocation of wealth across the assets. This is an especially relevant consideration as the number of assets grows large since statistical precision typically deteriorates in higher dimensions. With this in mind we assess the performance of an equal distribution of the portfolio wealth at each re-estimation. ${ }^{14}$ Call this the $1 / N$ benchmark, and note that while the turnover of this allocation strategy is low it is not, nor should it be, zero. The performance measures in Table 3.3 makes clear that there

Table 3.3: Performance of $1 / \mathrm{N}$ portfolio

\begin{tabular}{lccr|rrrrrrr}
\hline \hline & \multicolumn{3}{c}{$\tilde{t}=90$} & \multicolumn{3}{c}{$\tilde{t}=150$} & \multicolumn{3}{c}{$\tilde{t}=250$} \\
& $\hat{\sigma}$ & $\hat{\mu} / \hat{\sigma}$ & \multicolumn{1}{c}{$\hat{\delta}$} & $\hat{\sigma}$ & $\hat{\mu} / \hat{\sigma}$ & $\hat{\delta}$ & $\hat{\sigma}$ & $\hat{\mu} / \hat{\sigma}$ & \multicolumn{1}{c}{$\hat{\delta}$} \\
\hline Monthly & 17.54 & 0.51 & 2.55 & 17.04 & 0.62 & 2.53 & 17.16 & 0.59 & 2.54 \\
Quarterly & 17.51 & 0.51 & 2.55 & 16.92 & 0.64 & 2.53 & 17.13 & 0.59 & 2.53 \\
& $\chi_{\kappa=1}$ & $\chi_{\kappa=5}$ & $\chi_{\kappa=10}$ & $\chi_{\kappa=1}$ & $\chi_{\kappa=5}$ & $\chi_{\kappa=10}$ & $\chi_{\kappa=1}$ & $\chi_{\kappa=5}$ & $\chi_{\kappa=10}$ \\
\hline Monthly & -2.22 & 3.26 & 10.10 & -2.30 & 2.71 & 8.97 & -2.07 & 2.71 & 8.68 \\
Quarterly & -3.31 & 1.99 & 8.60 & -3.97 & 0.85 & 6.87 & -2.97 & 1.72 & 7.58 \\
\hline
\end{tabular}

The volatilities are denoted $\hat{\sigma}$, Sharpe ratios are $\hat{\mu} / \hat{\sigma}$, turnover is $\hat{\delta}$ and $\chi_{\kappa}$ is the estimated equalizing fee. All entries are annualized, the measures $\hat{\sigma}$ and $\chi_{\kappa}$ are multiplied by 100 .

are significant gains in variance reduction and Sharpe performance to be gained from a more involved estimation scheme. The volatility of our Factor-Graphical allocation is only 35 percent of the volatility achieved by the $1 / N$ benchmark. As for the Sharpe ratio, our method more than doubles the performance of the $1 / N$ portfolio. The caveat is predictably in the occurred transaction costs. The Factor-Graphical portfolios have higher turnover and are therefore more costly to maintain. In terms of investor utility we again find that risk averse actors still fa-

\footnotetext{
${ }^{14}$ The same rule of missing returns as applied to the estimators is also applied to the equally weighted allocation. If an asset is assigned a zero weight due to missing historical values it is also omitted from the equally weighted portfolio.
} 
vor the Factor-Graphical method. These investors accept very high fees, between 6 and 10 percent, on the Factor-Graphical portfolio before becoming indifferent. The investors with medium levels of relative risk aversion also favour the FactorGraphical portfolio. The low risk aversion investors prefer the equally weighted portfolio. Comparing the implied indifference fee of Table 3.2 to the results reported in Table 3.3, it is clear that the portfolio delivering the highest utility for low risk aversion investors is the $1 / N$ portfolio. For these investors, direct inverse estimation only outperforms the $1 / N$ allocation under the largest available window length, in which case they prefer the nodewise $\ell_{1}$ portoflio allocation.

In sum, we have found that the method we propose in this chapter appears to have strong risk reduction properties. The Factor-Graphical method reduces risk beyond alternative inverse estimations, nodewise ridge and lasso, as well as the main covariance estimators of the literature, the Shrinkage and the POET. Bringing all measures together into investor utility we find that the risk averse actors tend to prefer our Factor-Graphical, or the nodewise implementation of our method, despite the higher trading costs. Low risk aversion investors, however, tend to favor the nodewise allocation methods or the equally weighted portfolio.

\subsubsection{Decomposing the Factor-Graphical portfolio}

The Factor-Graphical portfolio combines common factor models with sparse hedging portfolios to form the minimum variance allocation. In this section we explore how these two components emerge empirically and whether we can achieve a similar level of financial performance while restricting one of the two components.

Our first evaluation is with respect to the implementation of the factors. Do the factors induce sparsity in the inverse, and how are the hedging relations developing over time. The factors must be sufficiently strong in order to induce sparsity in the inverse matrix. To give an indication of the factor potential, while avoiding the statistical issues of high-dimensionality, we randomly select 10 assets of the S\&P500 observed over 24 months following a random date between January 1st 2010 and December 31st 2015. Estimating the idiosyncratic inverse under the Fama-French 5-factor model we find a clear attenuation of the off-diagonal elements. ${ }^{15}$ Repeating this random selection and estimation $L$ times provides two smooth densities for the estimates absolute off-diagonal elements.

\footnotetext{
${ }^{15}$ The estimate of the inverse is based on the sample covariance matrix which works well for this purpose since the number of asset is very small.
} 
Figure 3.3: Off-diagonal elements $\Theta$ and $\Sigma^{-1}$

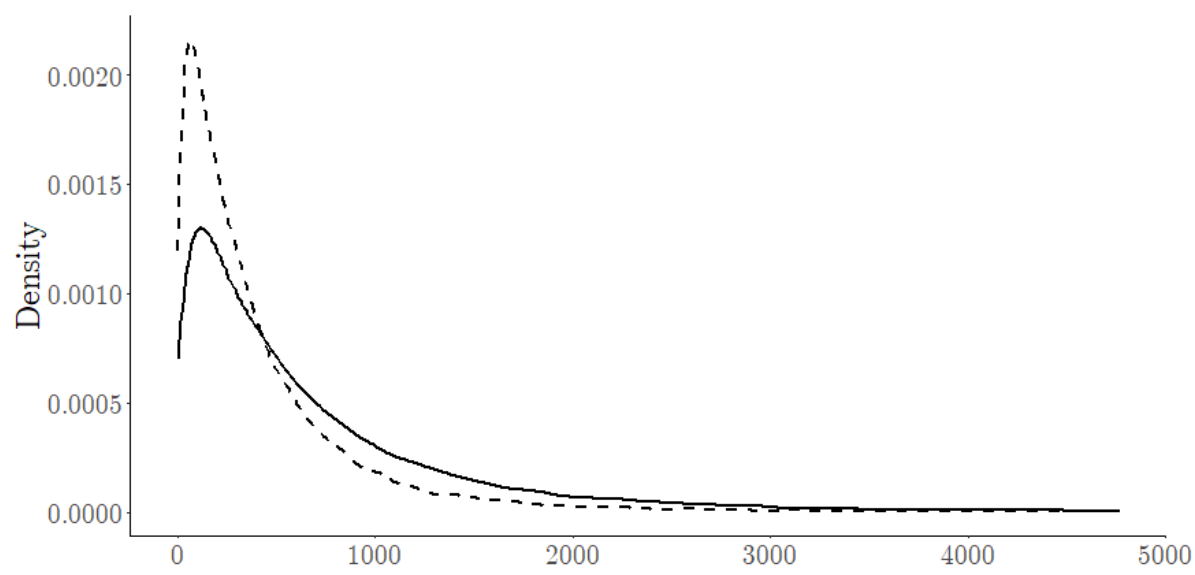

Empirical density of absolute off-diagonal elements from low-dimensional estimates of $\Theta$ and $\Sigma^{-1}$. Off-diagonal elements of idiosyncratic inverse $\widehat{\Theta}$ (dotted) and off-diagonal elements of $\widehat{\Sigma}^{-1}$ (solid). The two inverse covariance matrices are estimated based on historical financial returns. One thousand times, ten assets are randomly selected from the S\&P500 and observed during 24 months period with a random start date between 2010-01-01 until $2015-12-31$.

Figure 3.3 displays the densities of the absolute off-diagonal elements of the two inverses. The factors induce a shift in the distribution of hedging relations, hence aiding our sparsity assumption.

The second question we address is how the level of sparsity varies over time. We estimate the inverse covariance matrix monthly with the graphical lasso. Each of the estimates provides hedging portfolios for every asset. Allowing three estimation windows of 90,150 and 250 observations we find that the estimator tends to omit between 85 and 100 percent of the possible hedging coefficients. In the larger sample of 250 historical returns we find the most stable level of sparsity with between 5 and 10 percent of the available hedging relations used. Figure 3.4 displays the fraction of hedging coefficients that are set equal to zero by the graphical lasso.

If the fraction is consistently equal to one the estimator is simply reduced to a strict factor structure. However, with the largest estimation window there is only one instance in which the estimator omit all possible hedging relations. Under smaller estimation windows this is a much more frequent event, but the sparsity generally varies within the range of 85 to 100 percent. In all this implies that a 
Figure 3.4: Hedging portfolio sparsity over time

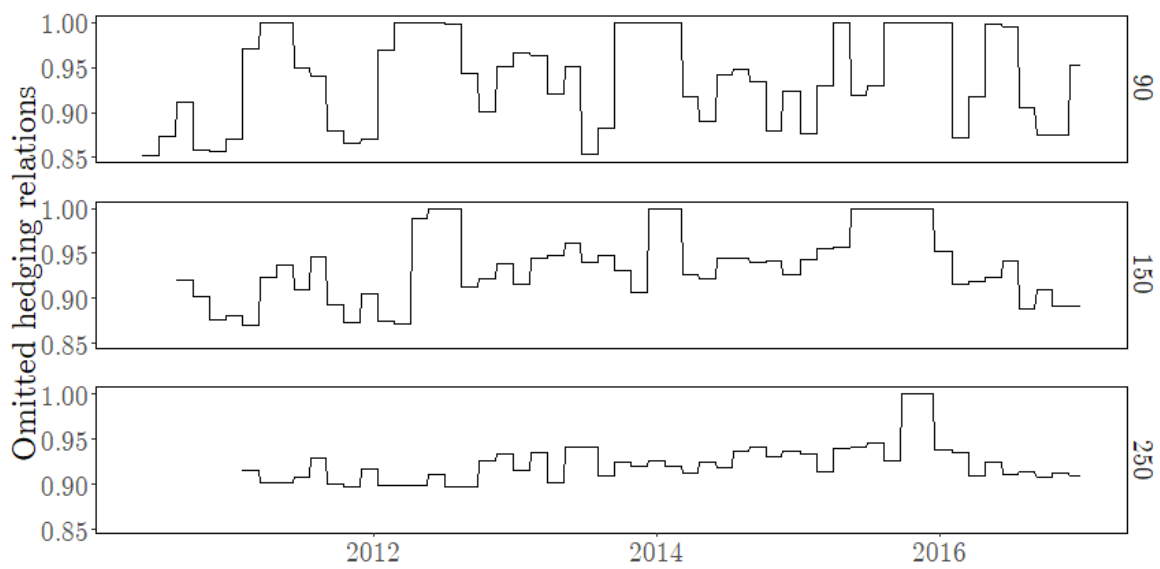

Fraction of hedging coefficients omitted by the graphical model selection under 3 sample windows, $\tilde{t}=\{90,150,250\}$. The total number of potential hedging relations is $N(N-1) / 2$. The graphical lasso performs model selection to set the irrelevant relations equal to zero.

strict factor structure may be too simple.

In the same way we follow the fraction of hedging relations in the whole portfolio, we also track the distribution of hedges per asset. Figure 3.5 display the median along with the 10th and 90th percentile of hedging relations per asset. The median number hedges per asset varies largely between 65 and 0 , when the estimation window consists of 250 observations. The period of zero included relations is during the last months of 2015 and corresponds to the estimate where all offdiagonal elements in the inverse were set to zero.

The shaded area of Figure 3.5 denotes the interval between, and including, the 10th and 90th percentile. From this plot we find that the number of assets in the hedging portfolios largely varies between 80 and 25 . Apart from the anomaly of the last months of 2015 it appears that the estimated optimal number of assets necessary to hedge the residual risk is largely stable.

Next we evaluate whether the Factor-Graphical performance stems from the combination of factors and the sparse hedging. The alternative would be that one of the facets singularly drive the risk reduction. We introduce two restricted alternatives to our method. First, a strict factor portfolio, where the estimate is based on a linear combination of the factor covariance matrix, scaled by the asset risk-loadings, and a diagonal residual covariance matrix. Second, a sparse hedging 
Figure 3.5: Distribution of assets per portfolio over time

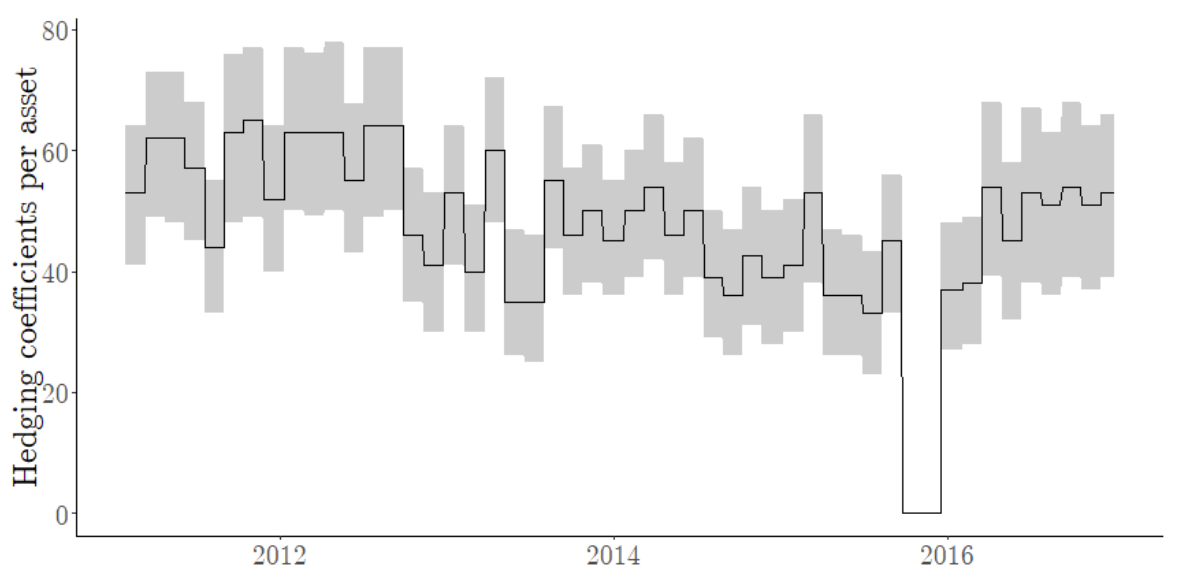

Number of hedging relations per asset. Based on estimate using window $\tilde{t}=250$. The solid line denotes the median number of hedging relations selected by the graphical model selection procedure. The shaded area displays the range between the 10th and 90th percentile of the number of hedging relations selected for an asset.

portfolio, which is based on a graphical lasso estimate but does not partition or make use of any common factor structure. This portfolio is allocated using the graphical lasso to directly estimate the inverse covariance matrix of financial returns, the marginal inverse discussed in Section 3.2. We refer to the first portfolio as the Factor and the second as the Graphical allocation.

The outcome of this comparison is summarized in Table 3.4, with estimates that are updated monthly and quarterly. The comprehensive Factor-Graphical method achieves the lowest portfolio volatility and outperforms the restricted versions. Relative to the Graphical allocation under an estimation window of 90 observations the portfolio volatility is lowered by an almost 40 percent and the Sharpe ratio is increased by almost 75 percent. The performance gains remain large as the sample window grows larger. The Factor-Graphical method also produce higher Sharpe ratios.

Between the restricted versions, the Factor allocation reduce volatility to a greater extent than the Graphical allocation. The level of risk reduction is larger under the smaller estimation window, however the difference between the two allocation rules appear to decrease as the estimation window grows larger. Using monthly estimates the Graphical portfolio fails to reach the Sharpe ratio of the 
Table 3.4: Restricted methods financial performance 2010-03-31 until 2016-12-28

\begin{tabular}{lrcc|ccccccr}
\hline \hline & \multicolumn{3}{c}{$\tilde{t}=90$} & \multicolumn{3}{c}{$\tilde{t}=150$} & \multicolumn{3}{c}{$\tilde{t}=250$} \\
\multicolumn{1}{c}{ Monthly } & \multicolumn{1}{c}{$\hat{\sigma}$} & $\hat{\mu} / \hat{\sigma}$ & $\hat{\delta}$ & $\hat{\sigma}$ & $\hat{\mu} / \hat{\sigma}$ & $\hat{\delta}$ & \multicolumn{1}{c}{$\hat{\sigma}$} & $\hat{\mu} / \hat{\sigma}$ & \multicolumn{1}{c}{$\hat{\delta}$} \\
\hline Factor-Graphical & 6.09 & 1.22 & 6.53 & 6.57 & 1.51 & 8.42 & 7.65 & 1.43 & 10.50 \\
Factor & 7.18 & 1.11 & 5.67 & 8.62 & 1.28 & 7.31 & 10.19 & 1.22 & 9.14 \\
Graphical & 10.06 & 0.72 & 2.94 & 9.87 & 0.99 & 3.55 & 10.40 & 1.03 & 4.17 \\
$\quad$ Quarterly & & & & & & & & & \\
\hline Factor-Graphical & 6.70 & 0.97 & 6.76 & 6.95 & 1.23 & 8.18 & 7.85 & 1.27 & 10.28 \\
Factor & 7.73 & 0.75 & 5.78 & 8.77 & 1.00 & 7.09 & 10.12 & 0.96 & 8.92 \\
Graphical & 10.12 & 0.70 & 3.15 & 9.97 & 0.98 & 3.53 & 10.46 & 0.96 & 4.19 \\
\hline
\end{tabular}

The volatilities $\hat{\sigma}$ and Sharpe ratios $\hat{\mu} / \hat{\sigma}$ are both annualized through a $\sqrt{252}$ scaling. Both the portfolio turnover $\hat{\delta}$ and the volatility $\hat{\sigma}$ are scaled by 100 .

The Factor portfolio allocation is based on the covariance matrix under the FamaFrench 5-factor model along with a diagonal residual variance matrix.

The Graphical portfolio is formed on an estimator that omits the factor structure. Here the graphical lasso is implemented directly on the financial return.

Factor allocation, but does exhibits much lower turnover than both the Factor allocation and the Factor-Graphical allocation. Hence, large transaction costs favor the Graphical portfolio while low costs is good for the allocation based on the common factors.

Table 3.5: Estimate of implied equalization fee $\left(\chi_{\kappa}\right)$

\begin{tabular}{lccccccccr}
\hline \hline & \multicolumn{3}{c}{$\tilde{t}=90$} & \multicolumn{3}{c}{$\tilde{t}=150$} & \multicolumn{4}{c}{$\tilde{t}=250$} \\
Monthly & $\chi_{1}$ & $\chi_{5}$ & \multicolumn{1}{c}{$\chi_{10}$} & $\chi_{1}$ & $\chi_{5}$ & $\chi_{10}$ & $\chi_{1}$ & $\chi_{5}$ & \multicolumn{1}{c}{$\chi_{10}$} \\
\hline Factor & -0.93 & -0.64 & -0.27 & -1.53 & -0.90 & -0.11 & -2.04 & -1.12 & 0.02 \\
Graphical & -1.31 & -0.01 & 1.61 & -2.07 & -0.97 & 0.41 & -2.76 & -1.76 & -0.50 \\
Quarterly & & & & & & & & & \\
\hline Factor & 0.31 & 0.61 & 0.99 & -0.63 & -0.05 & 0.68 & -0.29 & 0.53 & 1.57 \\
Graphical & -2.12 & -0.95 & 0.50 & -3.32 & -2.29 & -0.99 & -2.90 & -1.93 & -0.72 \\
\hline
\end{tabular}

The estimated constant daily fee sufficient to equate the Factor-Graphical portfolio with the competing methods. A negative value implies that the equalizing fee is imposed on the competing method, while a positive entries imply that the fee is put on the Factor-Graphical portfolio. As such, negative values imply investor utility under the competing method exceeds that of the Factor-Graphical portfolio. All the table entries are multiplied by 100.

Estimating the equalizing fees between the restricted methods and the method we propose, we again find that the Factor-Graphical portfolio more often than not provides higher utility when investors are more risk averse. But the differences are now very small and the outcomes are therefore mixed. Thus, the additional risk reduction under the Factor-Graphical allocation is here not always sufficient to offset the increased costs. 
In all it appears that the performance of our method is not driven by any of the respective components alone. The strict factor structure is generally to restrictive as the sample window increase. The Graphical allocation alone does not manage to reduce the portfolio volatility to any extent that is comparable to our Factor-Graphical method.

\subsubsection{Investor utility and performance under re-sampling}

So far we have estimated and compared the relative performance of our FactorGraphical method. We have not yet assessed the uncertainty in the performance and whether differences between methods are statistically significant. In order to do this testing we need an estimate of the standard errors of the respective measures. To assess the standard errors of the portfolio performance we evaluate the method under repeated re-sampling. Through this procedure we find that the increased risk reduction of our method is very statistically robust.

We set up this bootstrapping procedure such that we mimic the empirical challenge that an investor is faced with when maintaining a minimum variance portfolio. The observed returns are re-sampled of a block-wise approach, where the blocks are overlapping and with a fixed block length. Randomly sampling dates with their corresponding blocks we form series $T=1000$ observations in total but maintain the same cross-sectional dimensions as before. We set the block length $b$ equal to 50 while the estimators are given a sample window of either 90,150 or 250 observations. ${ }^{16}$

We let the investor form the respective portfolios and update their estimates after a predetermined time. Each estimate is evaluated against the dates that follow until the next estimation update. We vary the period between estimation updates in accordance with the block length such that we always evaluate against a single block. For example, when block length is $b=50$ the estimate is also updated every 50 days. If the sample window is set to $\tilde{t}=150$ this means the estimator estimates an allocation over three blocks then evaluates against one, after which it updates the estimates and the portfolio again until the end of the $T$ periods.

This procedure is repeated $L$ times, each time assessing the performance under the re-sampled data. In this way we gain a bootstrapping distribution for each of our metrics including the investor utility. Due to computational costs we restrict

\footnotetext{
${ }^{16}$ We find that varying the block length does not strongly affect the results.
} 
the evaluation to the Factor-Graphical method and the Shrinkage method. We choose the Shrinkage estimator because of its clear methodological differences to our method. Both the Shrinkage and the Factor-Graphical estimator are able to exploit factor structures, but the Shrinkage estimator is a covariance estimator and does not promote sparsity.

We adjust our graphical portfolio allocation slightly compared to the empirical application. In this section we choose the level of regularization, $\Lambda$, through a 3 -fold cross-validation over a reduced number of values, ten values on a linear grid on log scale. This change is due to the computational cost of the cross-validation. Apart from this adjustment in the graphical approach the methodology stays the same. The target matrix of the Shrinkage estimator is the covariance matrix implied by the Fama-French 5 -factor model with a diagonal residual covariance. In the same way we use the 5 -factor model to partition the covariance matrix for the graphical portfolio in accordance with partitions (3.4) and (3.6).

We record which method performs best in each respective sample and compute the relative frequency of times the Factor-Graphical method out performs the Shrinkage portfolio. The relative frequencies under all sample windows are summarized in Table 3.6. We set both the number of repetitions, $L$, and the number of periods $T=1000$.

Table 3.6: Factor-Graphical compared to Shrinkage

\begin{tabular}{lcccccc}
\hline & \multicolumn{3}{c}{ Performance } & \multicolumn{2}{c}{ Utility } \\
$\tilde{t}$ & $\hat{\sigma}$ & $\hat{\mu} / \hat{\sigma}$ & $\hat{\delta}$ & & $U_{\kappa=1}$ & $U_{\kappa=10}$ \\
\hline 90 & 1.00 & 0.55 & 0.00 & 0.44 & 0.59 \\
150 & 1.00 & 0.53 & 0.00 & 0.40 & 0.59 \\
250 & 1.00 & 0.48 & 0.01 & 0.39 & 0.54 \\
\hline
\end{tabular}

Relative frequency of samples in which the FactorGraphical method out performs the Shrinkage method. The length of the holding period and the block length set to 50 . Number of re-samplings is $L=1000$ and each contains $T=1000$ observations. Volatilities denoted $\hat{\sigma}$, Sharpe ratios are $\hat{\mu} / \hat{\sigma}$, turnover is $\hat{\delta}$. The investor utilities are denoted $U_{\kappa=1}$ and $U_{\kappa=10}$ respectively.

Consistently across re-sampling we find that the Factor-Graphical method reduces volatility more than the Shrinkage method. The volatility reduction performance in favor of the Factor-Graphical approach is therefore significant. Inversely, the Shrinkage portfolio maintains lower turnover in almost all samples. The Sharpe performance however, appears evenly split between the two methods. 
Turning to the investor utility we find that the distinction between the two methods is split by the the risk aversion parameter. We find that in low risk aversion case the Shrinkage portfolio tends to perform better. Out of the $L$ samples the method generates higher investor utility in around 60 percent of the samples. The fraction increases slightly as the sample window grows larger. In the high risk aversion setting we find the reverse. With a window of 150 observations the Factor-Graphical portfolio reduces the portfolio volatility enough to out-weight the transaction costs in almost 60 percent of the re-sampled datasets. As the sample window grows the relative frequency samples that favor this method decreases.

We summarize each of the bootstrapped performance distributions by the average difference and the average dispersion of differences across out $L$ samples in Table 3.7. The average volatility reduction is between 1.01 and 1.94 percentage points larger on average when using our Factor-Graphical method. The differences we find under the re-sampling vary on average by 0.33 percentage points under the smallest estimation window, and by 0.60 in the largest. Hence, the volatility reduction is not just statistically significant it is also economically very large for these assets.

Table 3.7: Performance difference between Factor-Graphical and Shrinkage.

\begin{tabular}{lrrrrrr}
\hline \hline & \multicolumn{2}{c}{$\tilde{t}=90$} & \multicolumn{2}{c}{$\tilde{t}=150$} & \multicolumn{2}{c}{$\tilde{t}=250$} \\
& Avg. & S.E. & Avg. & S.E. & Avg. & S.E. \\
\hline$\hat{\sigma}-\tilde{\sigma}$ & -1.01 & 0.33 & -1.64 & 0.41 & -1.94 & 0.60 \\
$\hat{\mu} / \hat{\sigma}-\tilde{\mu} / \tilde{\sigma}$ & 1.66 & 24.14 & 2.13 & 28.48 & -0.91 & 32.41 \\
$\hat{\delta}-\tilde{\delta}$ & 2.79 & 0.76 & 3.05 & 0.93 & 3.11 & 1.23 \\
\hline$\hat{U}_{\kappa=1}-\tilde{U}_{\kappa=1}$ & -0.92 & 4.85 & -1.36 & 5.33 & -1.78 & 5.31 \\
$\hat{U}_{\kappa=10}-\tilde{U}_{\kappa=10}$ & 0.15 & 0.88 & 0.21 & 0.97 & 0.13 & 0.96 \\
\hline
\end{tabular}

The performance of the Factor-Graphical portfolios are denoted $\hat{\sigma}, \hat{\mu}$ and $\hat{\delta}$, Shrinkage performance are denoted $\tilde{\sigma}, \tilde{\mu}$ and $\tilde{\delta}$. The Utility sums are denoted in the same way. Performance measures are annualized, and both mean and standard errors are multiplied by 100. The length of the holding period and the block length set to 50 . Number of re-samplings is $L=1000$ and each contains $T=1000$ observations.

\subsection{Conclusions}

In this chapter we propose a method of large scale portfolio optimization that builds upon the usage of factor models and high-dimensional statistics. The contribution to the literature comes from the combination of three features: (1) common 
factor models to account for commonalities; (2) high-dimensional model selection of hedging portfolios to compensate for factor misspecification; (3) direct inverse estimation to avoid explicit high-dimensional matrix inversion. Evaluating the method using a minimum variance allocation of S\&P500 companies we find that our method reduces portfolio volatility more than the competing alternative methods. We find that the performance gains are particularly large when the estimators are only allowed a small sample of historical returns.

High-dimensional portfolio allocation creates the need to estimate a highdimensional inverse covariance matrix. The method we propose adds to the large literature of methods that attempt to tackle this problem statistically (Ledoit and Wolf, 2003; Fan et al., 2008, 2013, 2015; Goto and Xu, 2015). Our method estimates this inverse through a partition on the inverse induced by a factor model. Partitioning the returns creates a large block of the inverse matrix that is sparse. The method estimates this block directly using the graphical lasso of Yuan and Lin (2007) and algorithm from Friedman et al. (2008). The advantage of this approach is that it circumvents the issues associated with high-dimensional matrix inversion. The sparse high-dimensional block joined with the factor model provides an estimate of the inverse covariance matrix of returns. From an economic perspective our method combines common factors, like the Fama-French factors, with a data-driven hedging selection to adjust for misspecification in the factor model.

We evaluate the method on a data set consisting of companies that at some point since 2005 were part of the S\&P500 index. Observing these assets on a daily frequency over seven years following 2010 we optimize the portfolio either every month or every quarter. The portfolio allocation we explore is a minimum variance strategy over three estimation windows, 90, 150 or 250 days. We compare the performance of our Factor-Graphical method against other closely related estimators and the principal methods of the literature. Empirically our Factor-Graphical method reduces the volatility of the minimum variance allocation more than any of the competing methods. Under the broader utility measures that account for average returns and trading costs, we find that our method is often favored among investors with high risk aversion.

The method we have proposed suggests several routes for additional work. It is well known that financial volatilities and covariances are highly persistent. What this implies and how to best incorporate this in the direct inverse estimate remains an open question. Another important decision is the choice of estimation window 
as well as re-estimation frequency. In this chapter we take these parameters as given but we also find that these choices are highly relevant for the portfolio performance. Future research should consider methods of applying dynamic weights to the sample. Beyond these tracks there are also open and interesting questions about the parsimonious factor model. There is by now an extensive literature proposing a different factors. Cochrane (2011) asks which factors provide independent information about expected returns. For our purposes this question translates into; which factors contribute to a partition that promotes sparsity in the hedging portfolios? This opens an additional stage of model selection. 



\section{Appendix}

\section{A Simulation design}

To illustrate the difference between estimating the inverse of the marginal covariance matrix and the inverse of the idiosyncratic inverse we set up a simple simulation study. We use Equation (3.3) to represent the returns of a set of $N$ assets.

$$
y=\boldsymbol{B} f+e, \quad f \sim \mathrm{N}(0, \Psi), \quad E \sim \mathrm{N}(0, \Delta), \quad B \sim \mathrm{U}_{(1,2)},
$$

where $y$ denotes a $N$-vector of returns, and $f$ is a $K$-vector of factors. The loading matrix, $B$, is $N \times K$. The covariance matrix of returns is $\Sigma=\boldsymbol{B} \Psi \boldsymbol{B}^{\prime}+\Delta$, with elements $\sigma_{i j}$. For our purposes we set the number of factors to $K=20$. The factor covariance matrix $\Psi$ is a diagonal matrix with variances drawn from a uniform probability distribution from 1 to $4.5, \operatorname{diag}(\Psi) \sim \mathrm{U}(1,4.5)$. We select these values based on the standard deviations of the five, daily, Fama and French factors observed from from 2005 until 2015. We take the asset specific sensitivities, $\boldsymbol{B}$, from a uniform distribution from 0 to 2 . At last, we calibrate the covariance matrix of the error terms $\Delta$. We consider a diagonal matrix where the univariate fit of the factor model provides an $R^{2}$ equal to 0.5 , hence the error variances are $\delta_{i i}^{2}=\left(\sigma_{i i}^{2}-0.5 \sigma_{i i}^{2}\right) / 0.5$. We draw $T=200$ observations from this framework.

We deviate from the strict factor structure and introduce misspecification through the error covariance matrix. We let the residual correlation matrix follow symmetric Toeplitz structure. The pairwise correlation coefficients decay linearly in the log-scale to $10^{-8}$. Hence, the correlation coefficients are all in the sequence 
$\rho_{p}=\exp \left(\ln (1)-\frac{(p-1)}{(N-1)} \ln \left(\frac{1}{10^{-8}}\right)\right)$ where $p=1 \ldots N$

$$
\left(\begin{array}{cccccc}
1 & \rho_{2} & \rho_{3} & \rho_{4} & \ldots & \rho_{n} \\
\rho_{2} & 1 & \rho_{2} & \rho_{3} & \ldots & \rho_{n-1} \\
\rho_{3} & \rho_{2} & 1 & \rho_{2} & & \vdots \\
\rho_{4} & \rho_{3} & \rho_{2} & 1 & & \vdots \\
\vdots & \vdots & & & \ddots & \vdots \\
\rho_{n} & \rho_{n-1} & \ldots & \ldots & \ldots & 1
\end{array}\right)
$$

This correlation structure implies that the inverse covariance matrix is tridiagonal, which means that the fraction of off-diagonal elements that are non-zero tends to zero as $N$ tend to infinity.

The important property is that the conditional correlation between assets is not all non-zero. Without that structure featuring sparse hedging portfolios is redundant beyond the common factors. Consider misspecification in the factor model. We refer to a well-specified model as a model that includes 19 out of 20 relevant factors. The severely misspecified model instead omits 19 relevant factors.

The idiosyncratic and marginal inverses are estimated using the graphical lasso,

$$
\operatorname{argmin}_{\Theta \succeq 0} f(\Theta)=-\log (\operatorname{det}(\Theta))+\operatorname{tr}(\widehat{S} \Theta)+\Lambda\|\Theta\|_{1},
$$

where $\widehat{S}=T^{-1} \widehat{E}^{\prime} \widehat{E}$, the covariance matrix of the residuals from (3.3) conditional on a set of factors. Both estimates require regularization parameters $\Lambda$. To reduce the computational cost of the simulations we calibrate the regularization using the extended Bayesian Information Criteria(eBIC) (Foygel and Drton, 2010). Denoting the number of non-zero entries in the matrix as $\mathbf{E}$ and $\psi \in[0,1]$ the information criteria is defined as,

$$
e B I C_{\psi}=-2 l_{T}(\Theta)+\mathbf{E} \log (T)+4 \psi \log (N) \mathbf{E},
$$

where inverse is denoted $\Theta$ and the sample covariance matrix $S$. The log likelihood of the inverse is $l_{T}(\Theta)=\frac{T}{2}(\log (\operatorname{det}(\Theta))-\operatorname{tr}(\Theta S))$. 
Figure 3.A.1: Mild misspecification; Fit of the estimates $\widehat{\Theta}$ to $\Delta^{-1}$

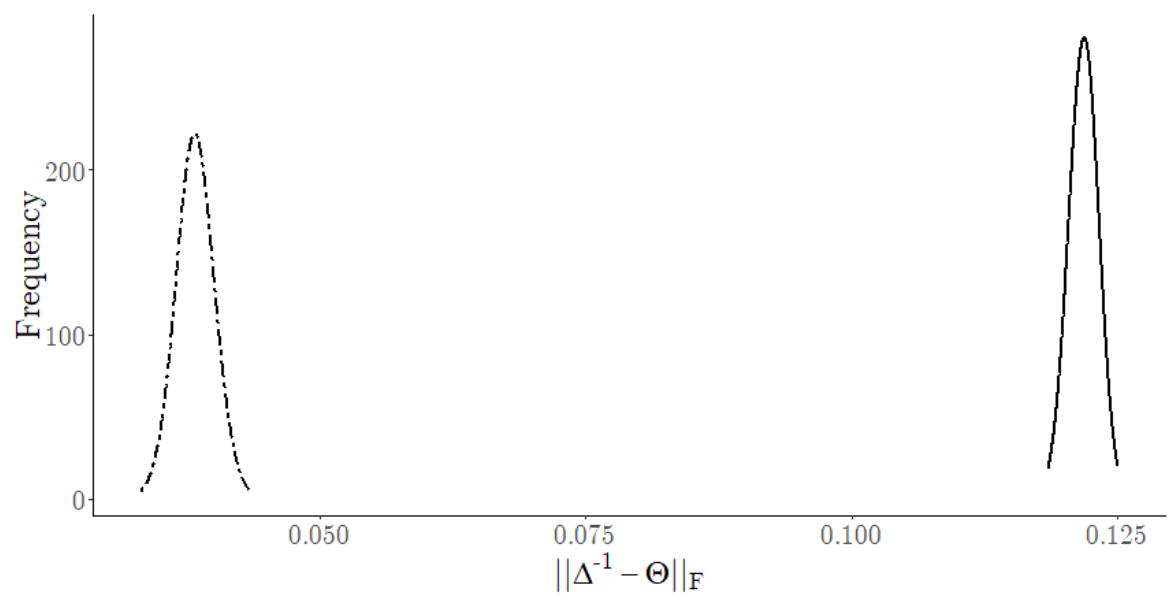

Frobenius norm of difference between the simulated idiosyncratic inverse $\left(\Delta^{-1}\right)$ and two estimates $(\widehat{\Theta})$. The estimate is either regularized with a $\ell_{1}$ or $\ell_{2}$ penalty. The solid line denotes the norm achieved using the $\ell_{2}$ regularization. The two-dashed line denotes $\ell_{1}$ regularization. Based on $L=1000$ simulations using a fixed design.

\section{Regularization: Shrinkage or Sparsity}

While the idiosyncratic inverse appears to be a good target for regularized estimation it is not clear what form of regularization should be implemented. One type of regularization is through the form of Shrinkage, where estimates are attenuated. However, in addition to attenuation we can regularize to set estimates exactly equal to zero, in other words regularization to promote sparsity. It is not obvious which form of regularization is best suited to estimate the idiosyncratic inverse when the factor model omits relevant factors.

To illustrate the issue consider the same simulation setup as before with 100 series each of 200 observations. The idiosyncratic inverse is approximated using two estimators, the first estimator induce shrinkage and sparsity, $\ell_{1}$ regularization, while the second estimator only induce shrinkage, $\ell_{2}$ regularization. To simplify the implementation we estimate the inverses using the neighbourhood selection approach of $N$ nodewise regularized regressions. We consider two cases, the first case is that of a mildly misspecified model and the second case is a model which is severely misspecified. The well-specified model omits 1 of the 20 relevant factors. This model is still misspecified but includes most relevant factors. The severely 
misspecified model omits 19 of the factors. Repeating the simulation $L$ times we compute the Frobenius norm of the difference between the estimated idiosyncratic inverse and the actual inverse. Figure 3.A.1 displays the norms of the two estimators, $\ell_{1}$ and $\ell_{2}$, using the factor model that omits one of the relevant factors. The level of regularization in the respective estimates is calibrated using 5-fold cross-validation.

The dashed line denotes the density of the sparse estimate while the dotted represents the shrinkage estimator. The regularization that promotes sparsity in the estimate of the idiosyncratic inverse also achieves a better fit to the actual inverse. In relation to the $\ell_{2}$ regularization the Frobenius norm is reduced by more than half. The second case, where the factor model omits 19 out of 20 factors is displayed in Figure 3.A.3.

Figure 3.A.2: Severe misspecification; Fit of the estimates $\widehat{\Theta}$ to $\Delta^{-1}$

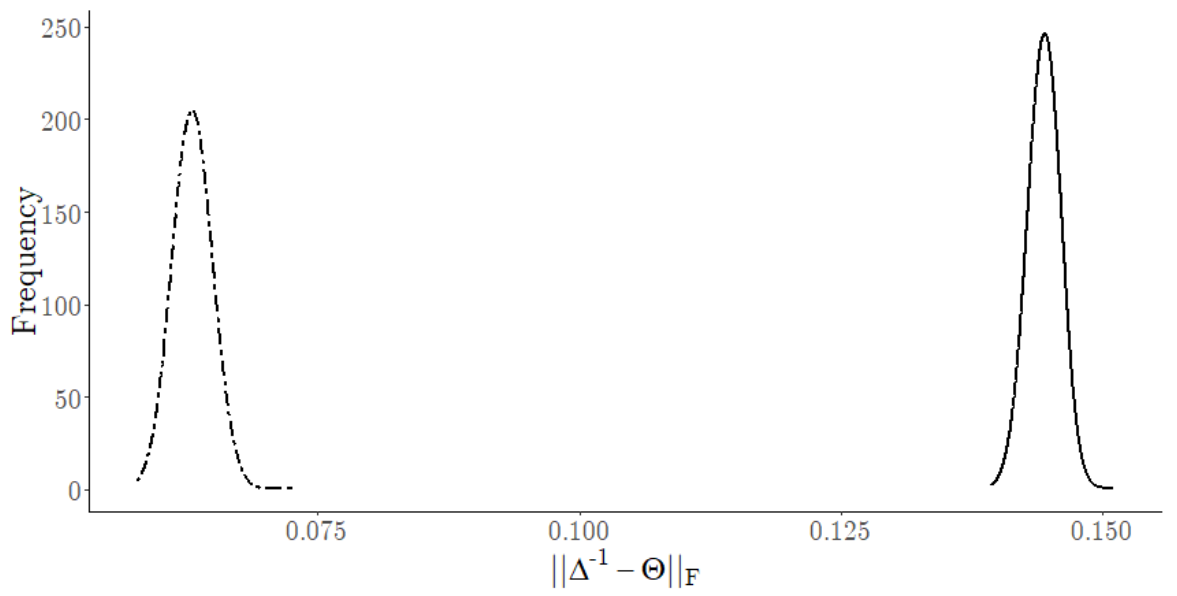

Figure 3.A.3: Severely misspecified factor model, nineteen omitted factors

Frobenius norm of difference between the simulated idiosyncratic inverse and two estimates $(\Theta)$. The estimate is either regularized with a $\ell_{1}$ or $\ell_{2}$ penalty on the off-diagonal elements. The solid line denotes the norm achieved using the $\ell_{2}$ regularization. The two-dashed line denotes $\ell_{1}$ regularization. Based on $L=1000$ simulations using a fixed design.

We find that the accuracy of the $\ell_{1}$ regularization strictly dominates the estimator using the $\ell_{2}$ penalization. The relative gain under the severally misspecified model compared to the first case is, however, smaller. In all it appears that imposing sparsity in the estimate in addition to shrinkage improves the accuracy of 
the estimated idiosyncratic inverse. This is found both when the factor model is severely misspecifed and when the model only omits one factor. These results guide us in the direction of the $\ell_{1}$ penalization but we have not yet evaluated the relative portfolio performance under the two estimators. Hence, for our central empirical analysis the main focus will be on regularizations that promote sparsity. Based on these results we expect these estimators to generate the best portfolio performance, but we shall also consider estimators under $\ell_{2}$ regularization.

\section{B S\&P500 financial data}

All the data we use for the empirical exercise is collected from CRSP. We restrict the sample to assets of companies that at some point were part of the S\&P500 companies between 2005 and 2017. This ensures that the stocks we allocate for our portfolios are of significant market capitalization and traded on a regular basis. Below follows basic descriptive statistics of the returns in excess to the risk-free rate including annualized volatilities and Sharpe ratios.

Table 3.B.1: Descriptive statistics of S\&P500 firms

\begin{tabular}{lrrrcccc}
\hline \hline \multicolumn{1}{c}{$\mathrm{N}=699$} & Mean & St.Dev. & Min. & Q1 & Q2 & Q3 & Max \\
\hline Returns & 0.10 & 0.22 & -2.73 & 0.05 & 0.11 & 0.16 & 1.46 \\
Volatilities & 0.32 & 0.14 & 0.02 & 0.23 & 0.28 & 0.35 & 1.97 \\
Sharpe Ratios & 0.42 & 0.62 & -2.91 & 0.19 & 0.41 & 0.58 & 7.00 \\
\hline
\end{tabular}

Descriptive statistics of the average annualized daily returns, annualized return volatility and annualized Sharpe ratios of the S\&P500 companies during 2010-2017. Mean refers to the arithmetic average, St.Dev the standard deviation, Q1, Q2 and Q3 denotes the first quartile, median and third quartile. The number of assets is denoted $N$.

The summary statistics displayed in Table 3.B.1 indicate that the annualized return volatilities range from 0.0222 to 1.9695 centered around 0.3091 . There is one asset with a Sharpe ratio of 7.0020. This firm is only observed in the first 32 days of the sample and can therefore not be included in any allocations.

Figure 3.B.1 shows the development of the index. It is clear that there have been significant positive development from 2012 to 2016, however, the years preceding and following that growth period saw significant volatility in the index return. 
Figure 3.B.1: S\&P500 Composite Index and Return, 2010-2017

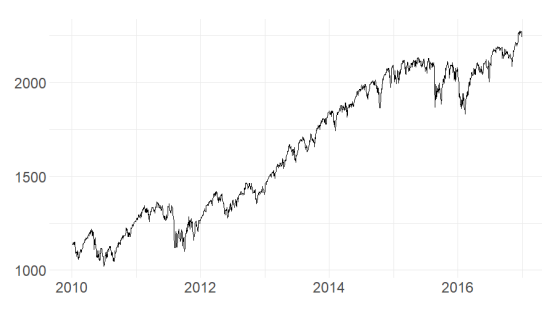

(a) Index in Level

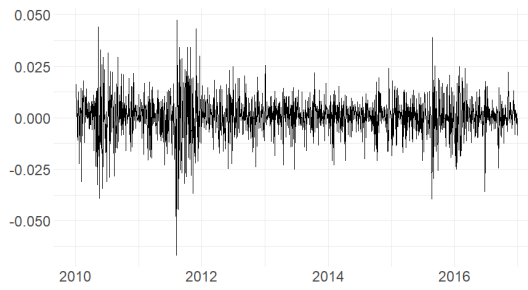

(b) Index Return

\section{C Competing estimators: POET and Shrinkage}

The shrinkage estimators of Ledoit and Wolf $(2003,2004,2012)$ are among the principal methodologies for high-dimensional covariance estimation. These estimators are based on the convex combination of the unconditional empirical covariance matrix and a prior target matrix. Here, the target is typically the covariance implied by a set of economic factors or, alternatively, a numerically well-behaved statistical construct. In this chapter we use a target matrix based on an economic model of observed factors, the Fama-French five factor model. The model implies that the covariance matrix can be decomposed into the covariance matrix, $\Psi$, of the factors subject to the asset specific loadings, and a diagonal residual variance matrix,

$$
\widetilde{\Sigma}=\boldsymbol{B} \Psi \boldsymbol{B}^{\prime}+\Delta
$$

The estimate $\widetilde{\Sigma}$ serves as the target covariance matrix towards which we shrink the sample covariance matrix $S$ by the shrinkage parameter $\pi$.

$$
\Sigma=\pi \widetilde{\Sigma}+(1-\pi) S
$$

The optimal shrinkage has to be estimated with an estimator derived by Ledoit and Wolf (2004) with respect to the squared Frobenius norm. With the estimate

$\widehat{\Sigma}$ we proceed by matrix inversion and minimum variance allocation such as it is defined in Equation (3.2).

In addition to the shrinkage estimator we also consider the method of Principal Orthogonal complEment Thresholding (POET) of Fan et al. (2013). This estimator serves in our application as a statistical factor model analogue to the 
shrinkage estimator. The POET methodology also builds upon a parsimonious latent structure of the asset returns, but expands particularly on the case where factors are unobserved. Let $\left[v_{1} \ldots v_{k}\right]$ and $\left[V_{1} \ldots V_{k}\right]$ denote the first $k$ eigenvalues and vectors of returns respectively. Choose the number of factors to include, $\hat{K}$, through the information criteria of Bai and $\mathrm{Ng}$ (2002a), as is standard in the literature,

$$
\hat{K}=\operatorname{argmin}_{1 \leq K \leq K^{*}}\left(\ln \left(\sum_{i>K} \hat{v}_{i}^{2}\right)+\frac{K(N+T)}{N T} \ln \left(\frac{N T}{N+T}\right)\right) .
$$

With the number of factors chosen we may express the covariance matrix as

$$
S=\sum_{i=1}^{\hat{k}} v_{i} V_{i} V_{i}^{\prime}+\sum_{i=\hat{k}+1}^{N} v_{i} V_{i} V_{i}^{\prime}
$$

The POET methodology replaces the latter term, $\widetilde{U}=\sum_{i=\hat{k}+1}^{N} v_{i} V_{i} V_{i}^{\prime}$ with the term $\widehat{U}$ defined,

$$
\widehat{U}=\left(\hat{u}_{i j}\right)_{N \times N}, \quad \hat{u}_{i j}= \begin{cases}\tilde{u}_{i j}, & i=j \\ s_{i j}\left(\tilde{u}_{i j}\right) & i \neq j\end{cases}
$$

One choice is to specify the function $s_{i j}$ as a soft thresholding rule where $s_{i j}=$ $\left(\tilde{u}_{i j}-\tau_{i j}\right)_{+}$, where the parameter $\tau_{i j}=\zeta\left(\tilde{u}_{i i} \tilde{u}_{j j}\right)^{1 / 2}$. The constant $\zeta$ is typically set to 0.5 when using soft thresholding rules, but can be varied over a grid until the final estimates matrix is well-conditioned. With the thresholding imposed on the residual covariance matrix we can estimate the covariance matrix of the returns.

$$
\widehat{\Sigma}=\sum_{i=1}^{\hat{k}} \hat{v}_{i} \hat{v}_{i} \hat{v}_{i}^{\prime}+\widehat{U}
$$

This estimate is non-singular and well-conditioned. This is clearly not a comprehensive overview of the literature regarding high-dimensional covariance estimation. However, these two methodological alternatives tend to be favored in the broader portfolio allocation literature and are therefore given priority here to contrast the performance of our Factor-Graphical estimator. 
Table 3.D.1: Portfolio composition summary statistics

\begin{tabular}{lrrrrrrrrr}
\hline \hline & \multicolumn{3}{c}{$\tilde{t}=90$} & \multicolumn{4}{c}{$\tilde{t}=150$} & \multicolumn{4}{c}{$\tilde{t}=250$} \\
Monthly & Range & \multicolumn{1}{c}{$p_{-}$} & $\sum w^{2}$ & Range & \multicolumn{1}{c}{$p_{-}$} & $\sum w^{2}$ & Range & \multicolumn{1}{c}{$p_{-}$} & $\sum w^{2}$ \\
\hline F-G & 29.71 & 34.88 & 17.27 & 21.94 & 35.26 & 13.31 & 11.83 & 35.47 & 8.48 \\
NW $\ell_{1}$ & 39.67 & 35.13 & 26.03 & 31.15 & 34.95 & 18.66 & 18.89 & 34.55 & 9.63 \\
NW $\ell_{2}$ & 41.13 & 35.34 & 28.56 & 31.14 & 34.75 & 19.43 & 19.82 & 34.47 & 9.80 \\
POET & 20.85 & 33.92 & 11.00 & 13.17 & 34.27 & 7.16 & 5.77 & 34.80 & 3.00 \\
Shrinkage & 27.82 & 34.49 & 16.43 & 20.28 & 34.68 & 12.88 & 10.98 & 34.74 & 7.64 \\
Quarterly & & & & & & & & & \\
\hline F-G & 27.96 & 34.71 & 16.14 & 23.66 & 35.22 & 14.73 & 12.69 & 35.61 & 8.77 \\
NW $\ell_{1}$ & 34.00 & 35.61 & 22.24 & 34.06 & 35.05 & 22.44 & 21.12 & 34.61 & 11.58 \\
NW $\ell_{2}$ & 40.50 & 35.59 & 28.10 & 36.23 & 34.92 & 23.61 & 21.35 & 34.72 & 11.49 \\
POET & 19.43 & 34.14 & 10.37 & 15.20 & 34.30 & 8.39 & 6.44 & 35.06 & 3.17 \\
Shrinkage & 25.66 & 34.13 & 15.20 & 22.47 & 34.63 & 14.60 & 12.19 & 34.88 & 8.35 \\
\hline
\end{tabular}

Nodewise $\ell_{1}$ and $\ell_{2}$ procedures are abbreviated NW. Here Range denotes the average difference between the smallest non-zero weight and the largest. The average proportion $(\%)$ of negative weights assigned by the estimator is denoted $p_{-}$and the mean sum of squared weights is $\sum w^{2}$. The columns $\sum w^{2}$ and Range are multiplied by 100 .

\section{D Portfolio composition}

Table 3.D.1 summarizes some of the basic characteristics of the portfolios, such as Range, Fraction of negative weights and Dispersion. The fraction of negative weights is the sum of negative weights divided by the number of weights assigned by the respective methods. ${ }^{17}$ The Range indicates the average difference between the smallest, non-zero, weight and the largest weight in the portfolio. This overview of the distribution supply lets us discover methods prone to taking extreme positions. We measure the dispersion of the portfolio through the sum of squared weights.

Consistently across the different estimation windows we find that the Shrinkage portfolios along with the POET allocations produce a smaller spectrum of weights. This is supported by both the average range and the measure of dispersion. The difference between the Shrinkage portfolio and the Factor-Graphical method is however very small. Hence, our method is only very marginally more prone to form portfolios with more extreme weights. For example, with 90 observations in the sample window, and re-estimating monthly, the range of the Factor-Graphical allocation is 29.71 percentage points, while Shrinkage portfolio range is 27.71 per-

\footnotetext{
${ }^{17}$ This is not the same as the faction of negative weights in the whole portfolio. Assets which contain missing values are automatically assigned a zero weight. These weights are omitted when we compute the faction of negative weights since including them may distort the results when we increase the estimation window.
} 
centage points. This difference decreases as the sample window grows. Nodewise lasso and nodewise ridge estimators however appear much more volatile in their composition. These portfolios appear as outliers with respect to their dispersion and range, far exceeding the other alternatives.

Through Table 3.D.1 we also find that the none of the methods appear more or less likely to produce negative weights. The fraction of negative weights is, for all methods, around 35 percent and largely stable across sample sizes and with only marginal differences between estimators.

\section{E Bootstrap distributions}

In Figures 3.E.1, 3.E.2 and 3.E.3 we display the distributions of annualized out-ofsample volatility, Turnover and Sharpe ratios across the $L$ re-sampled data sets. The dashed lines denote the distribution of the Factor-Graphical performance while the solid lines are the Shrinkage portfolios.

Figure 3.E.1: Volatility sampling distribution

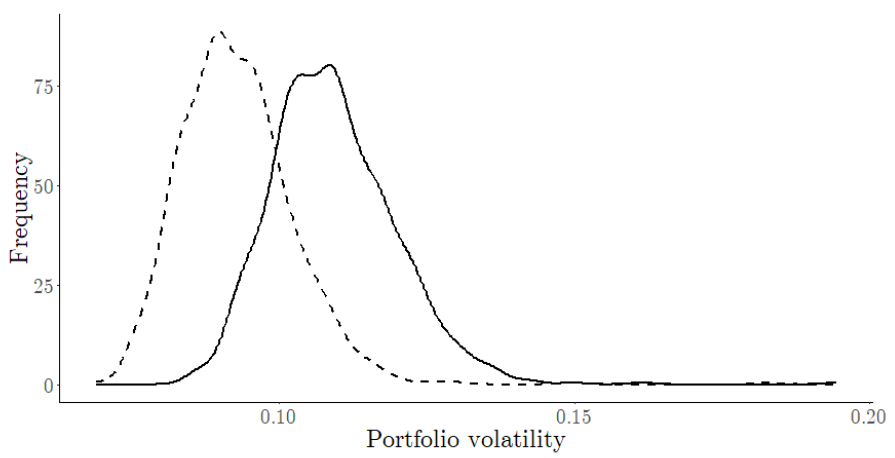

Portfolio Volatility, smoothed distribution over $L=1000$ re-sampled data sets. Each data have $T=1000$ observations re-sampled from the S\&P500 returns in a overlapping block-wise manner with block length, $b=50$. The solid line denotes the Lediot-Wolf shrinkage portfolios using the Fama-French 5 factor model as the target matrix. The dashed line denotes the graphical portfolios conditional on the same 5-factor model. All values are annualized.

The annualized volatility distribution, Figure 3.E.1, under the Factor-Graphical approach ranges from 0.061 to 0.191 with a median of 0.095 . The same distribution for the Shrinkage estimator is from 0.070 to 0.207 , median at 0.110 .

This shift in the volatility distribution in favor of the Factor-Graphical approach is reversed in the Turnover distribution, shown in Figure 3.E.2. The mean 
turnover over the bootstrap samples is higher for the Factor-Graphical portfolios. This reflect the same trade-off we found under the empirical application, the Factor-Graphical portfolio reduces volatility more than the Shrinkage portfolio, but requires more trading to keep the portfolio optimized upon re-estimation.

The Sharpe ratios are however very similar in their empirical distribution, Figure 3.E.3. The annualized Sharpe ratios varies between -1.5 and 2.5 with no discernible differences to favor one method over the other. As we turn to the investor utility that reflect all these aspect of financial performance we make use of two risk aversion settings. A low risk aversion case, with the risk aversion parameter $\kappa=1$, and a high risk aversion case $\kappa=10$. Transaction costs are 50 basis points under which we compute the sum of investor utility across the $L$ re-sampled datasets.

Figure 3.E.2: Turnover sampling distribution

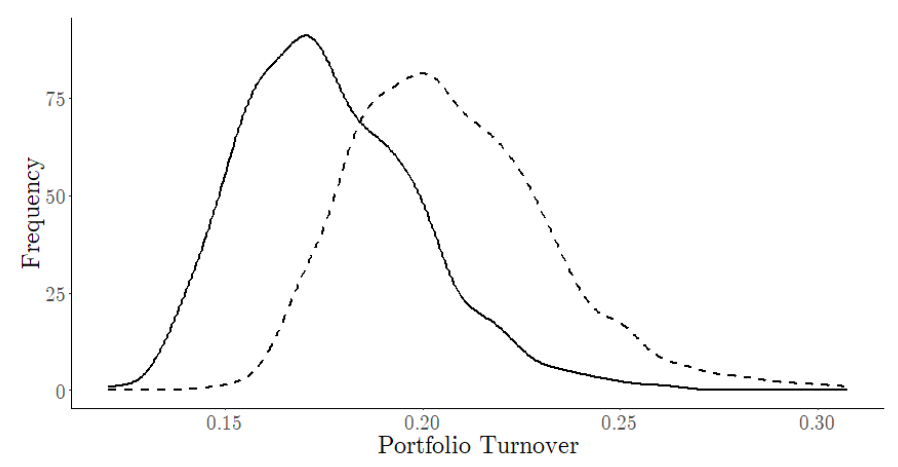

Portfolio Turnover, smoothed distribution over $L=1000$ re-sampled data sets. Each data have $T=1000$ observations re-sampled from the S\&P500 returns in a overlapping block-wise manner with block length, $b=50$. The solid line denotes the Lediot-Wolf shrinkage portfolios using the Fama-French 5 factor model as the target matrix. The dashed line denotes the graphical portfolios conditional on the same 5-factor model. All values are annualized.

The investor utility distribution are displayed in Figure 3.E.4. While the empirical utility distributions appear be centered around similar means the variance of the Factor-Graphical distribution is lower. Despite this it remains ambiguous which method generally performs better. 
Figure 3.E.3: Sharpe ratio sampling distribution

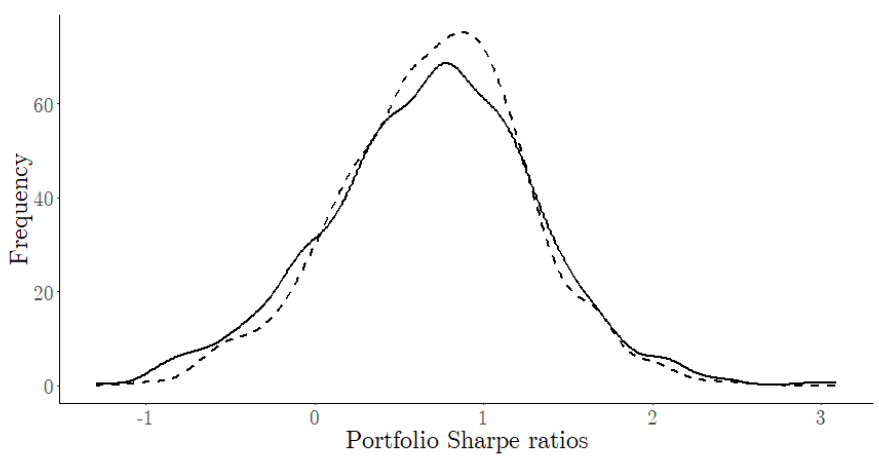

Portfolio Sharpe ratios, smoothed distribution over $L=1000$ re-sampled data sets. Each data have $T=1000$ observations re-sampled from the S\&P500 returns in a overlapping block-wise manner with block length, $b=50$. The solid line denotes the Lediot-Wolf shrinkage portfolios using the Fama-French 5 factor model as the target matrix. The dashed line denotes the graphical portfolios conditional on the same 5-factor model. All values are annualized.

Figure 3.E.4: Utility sampling distribution

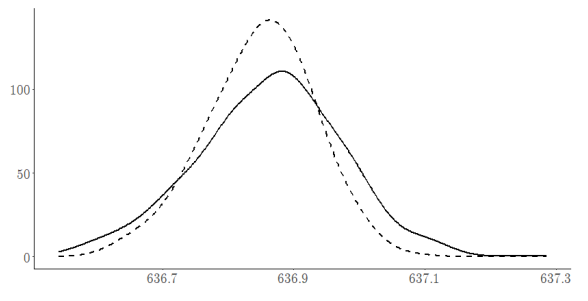

(a) Low risk aversion, $\kappa=1$

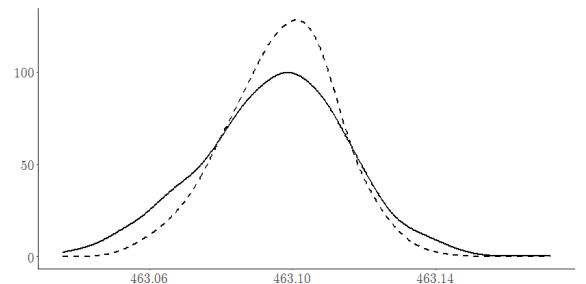

(b) High risk aversion, $\kappa=10$

Investor utility estimated under a quadratic utility function with adjustments for turnover. The solid line denotes the Lediot-Wolf shrinkage portfolio using the Fama-French 5 factor model as the target matrix. The dashed line denotes the graphical portfolio conditional on the same 5-factor model. We compute the utility following Equation (3.21) using two settings for the risk aversion, high and low. Transaction costs of 50 basis points are set to increase proportionally with the turnover. 


\section{F Conditional sparsity in the inverse}

We deviate from previous methods that emphasize conditional sparsity in the error covariance matrix by shifting the assumption to a conditionally sparse inverse covariance matrix. In this section we outline an example of an economic model under which our assumption provides a better representation than does the traditional sparse covariance assumption. We expand model (3.3) to include a set of $k$ unobserved local factors $f$ that provides smaller blocks of co-varying returns,

$$
y=\alpha+\boldsymbol{B} F+b f+u \text {. }
$$

The covariance matrix, $\Delta$, of the error term is diagonal. For simplicity assume that one asset only is exposed to one local factor. Thus, columns of the exposures $b$ contain many zeroes and multiple factors do not load onto the same asset. Those returns exposed to a local factor have a loading of one. Thus, $b$ contains only zeroes and ones, and we refer to them as local factors. We define the covariance matrix of the local factors as decaying in the off-diagonal elements such that,

$$
\Psi_{f}=\left(\begin{array}{cccc}
1 & c_{1,2} & \ldots & c_{1, k} \\
c_{2,1} & 1 & \ldots & c_{2, k-1} \\
\vdots & \vdots & \ddots & \vdots \\
c_{2, k} & c_{k-1,2} & \ldots & 1
\end{array}\right)
$$

Where $c_{i, j}=c^{|i-j|}$ where $i, j=1 \ldots k$ and $0<c<1$. This is known as a Kac-Murdock-Szegö matrix. Hence, assets are exposed to local factors where the factors are correlated but in a decreasing fashion. While the covariance decay along the off-diagonal elements, the inverse $\Psi_{f}^{-1}$ is tridiagonal. With this structure, for a small number of local factors, the covariance matrix $\Sigma_{e}=b^{\prime} \Psi_{f} b+\Delta$ is only approximately sparse as $c$ approaches zero. The inverse covariance matrix is decomposed as,

$$
\Sigma_{e}^{-1}=\left(b^{\prime} \Psi_{f} b+\Delta\right)^{-1}=\Delta^{-1}-\Delta^{-1} b\left(b^{\prime} \Delta^{-1} b+\Psi_{f}^{-1}\right)^{-1} b^{\prime} \Delta^{-1}
$$

The local factors create block structures within the financial assets. Since the correlation between the local factors is decreasing so is also the correlation 
between the blocks of assets. Ultimately, both $\Sigma_{e}$ and $\Sigma_{e}^{-1}$ are full matrices, however, the rates of decrease across the off-diagonal elements in the two matrices are potentially very different. The decay of the elements away from the diagonal is determined by the decay in the term $\left(b^{\prime} \Delta^{-1} b+\Psi_{f}^{-1}\right)^{-1}$,

$$
\left(b^{\prime} \Delta^{-1} b+\Psi_{f}^{-1}\right)^{-1}=\left(\begin{array}{cccc}
\sum b_{1}^{2}+\frac{1}{1-c^{2}} & \frac{-c}{1-c^{2}} & & \\
\frac{-c}{1-c^{2}} & \sum b_{2}^{2}+\frac{1+c^{2}}{1-c^{2}} & \ddots & \\
& \ddots & \ddots & \frac{-c}{1-c^{2}} \\
& & \frac{-c}{1-c^{2}} & \sum b_{N}^{2}+\frac{1}{1-c^{2}}
\end{array}\right)^{-1}
$$

The decrease across off-diagonal elements of different blocks in the covariance is constant at $c^{-1}$. In $\Sigma_{e}^{-1}$ the rate of decrease is non-constant. Following Meurant (1992) the non-constant rates of decay in this matrix is,

$$
\frac{\left(\left(b^{\prime} \Delta^{-1} b+\Psi_{f}^{-1}\right)^{-1}\right)_{i, j}}{\left(\left(b^{\prime} \Delta^{-1} b+\Psi_{f}^{-1}\right)^{-1}\right)_{i, j+1}}=\frac{\left(1-c^{2}\right) d_{j+1}}{c} .
$$

The term $d$. is the diagonal elements of a upper triangular decomposition, which here is expressed as a recursion along with a terminating condition,

$$
d_{i}=\left\{\begin{array}{ll}
\sum b_{i}^{2}+\frac{1+c^{2}}{1-c^{2}}-\frac{\left(c /\left(1-c^{2}\right)\right)^{2}}{d_{i+1}} & i<N \\
\sum b_{i}^{2}+\frac{1}{1-c^{2}} & i=N
\end{array} .\right.
$$

Following the results of Meurant (1992) we find that the elements of our inverse is strictly decreasing. Together the rate in (3.37) along with the term (3.38) gives us the rate of decrease in $\Sigma_{e}^{-1}$,

rate $_{j+1}=\frac{\left(\left(b^{\prime} \Delta^{-1} b+\Psi_{f}^{-1}\right)^{-1}\right)_{i, j}}{\left(\left(b^{\prime} \Delta^{-1} b+\Psi_{f}^{-1}\right)^{-1}\right)_{i, j+1}}=c^{-1}\left(1+\left(1-c^{2}\right) \sum b_{j+1}^{2}+c^{2}-\frac{c^{2} /\left(1-c^{2}\right)}{d_{j+1}}\right)$.

From this rate we find that the off-diagonal decay in the inverse covariance matrix always exceeds the decrease in the covariance matrix if $d_{j+1}>1 /\left(1-c^{2}\right)$. 
This clearly holds for $j=N-1$. With the rate of decrease in the covariance matrix of $c^{-1}$ we find,

$$
\text { rate }_{j+1}>c^{-1} \Longleftrightarrow d_{j+1}>\frac{1}{1-c^{2}}
$$

Starting from the end of the recursion we take $j=N-1$,

$$
d_{N-1}=\sum b_{N-1}^{2}+\frac{1+c^{2}}{1-c^{2}}-\frac{\left(c /\left(1-c^{2}\right)\right)^{2}}{d_{N}} .
$$

Applying the necessary inequality we find,

$$
\sum b_{N-1}^{2}+\frac{1+c^{2}}{1-c^{2}}-\frac{\left(c^{2} /\left(1-c^{2}\right)\right)^{2}}{d_{N}}>\frac{1}{1-c^{2}}
$$

which we can simplify the formulation to

$$
\left(1-c^{2}\right) \sum b_{N-1}^{2}+1+c^{2}-\frac{c^{2} /\left(1-c^{2}\right)}{d_{N}}>1
$$

This inequality holds if,

$$
c^{2}-\frac{c^{2} /\left(1-c^{2}\right)}{d_{N}}>1
$$

or put differently below,

$$
d_{N}>\frac{1}{\left(1-c^{2}\right)}
$$

Since $d_{N}>1 /\left(1-c^{2}\right)$ for all non-zero local exposures we have that $d_{N-1}>$ $1 /\left(1-c^{2}\right)$. Along the same lines for $j=N-2$,

$$
d_{N-2}=\sum b_{N-2}^{2}+\frac{1+c^{2}}{1-c^{2}}-\frac{\left(c /\left(1-c^{2}\right)\right)^{2}}{d_{N-1}}
$$


and,

$$
\left(1-c^{2}\right) \sum b_{N-2}^{2}+1+c^{2}-\frac{c^{2} /\left(1-c^{2}\right)}{d_{N-1}}>1
$$

we require,

$$
d_{N-1}>\frac{1}{\left(1-c^{2}\right)}
$$

Which we know holds from the previous case. Hence for all $j=1, \ldots, N$ the rate of decrease in the inverse covariance matrix exceed $c^{-1}$, the rate in the covariance matrix.

Furthermore, reviewing the off-diagonal decrease as a function of the parameter $c^{-1}$ we find that as $c$ tends to zero the rates in the two matrices is identical. However, as $c$ approaches one, the difference between the rates increase in favour of the inverse. This implies that while both the covariance and inverse covariance are full matrices, the elements of the inverse tend to zero faster as $N$ increase. Thus, the local factors of this form provides a circumstance where the sparsity assumption is more suitable in the inverse rather than the covariance matrix. Hence, a sparse estimate of the inverse covariance matrix is more suitable than a sparse representation of the covariance matrix. 



\section{Chapter 4}

\section{Pricing impacts of trade in a large cross-section of returns}

We estimate price-trade relations among a very large number of financial assets. Within an economic framework of informational asymmetry this representation provides insight into the liquidity of individual assets and the market. To accommodate the very large cross-section of assets we rely on linear regressions regularized using the elastic net. This allows the size of the asset neighborhoods to vary greatly between assets, which affords us greater flexibility in the estimation. The estimates can intuitively be thought of as a network where we summarize the asset level of illiquidity through its connectedness to the network. Forming portfolios based on individual asset connectedness, as well as sensitivity to the average connectedness, we find that this measure is priced and provides a return premium in excess of the Fama-French factors. ${ }^{1}$

\footnotetext{
${ }^{1}$ This chapter is based on the paper Basturk and Lönn (2018)
} 


\subsection{Introduction}

Empirical studies of financial liquidity present a number of prominent observations. Two important facts are that liquidity appears strongly time-varying and often exhibits commonalities across assets. In this chapter we propose a network representation to track liquidity developments. In particular we focus on pricing impacts within large cross-sections of assets. The network price-trade sensitivities we propose describes short-run relations across a vast number of assets. As the networks evolve over time, the estimated sensitivity describes the state of that asset, and through cross-sectional aggregation, the market. Sensitivity in our network parallels features of connectedness from the network literature. This interpretation provides an intuitive framework where assets that are highly connected assets to the network are more sensitive to order flow adjustments in neighboring assets. Such assets are less liquid and incur risk onto the investor. Empirically, we find that asset level connectedness is priced risk, significant in excess to the Fama-French factors. The associated return premium appears to have grown larger following the 1990s'. Further, estimates of the market level liquidity coincides with recent periods of financial distress.

We estimate the relative degree of price sensitivity through short-run tradepricing relations. Assets that display high sensitivity are as a consequence less financially liquid (Kyle, 1985). The empirical framework that we propose for the network estimates is a multivariate analogue of Pastor and Stambaugh (2003). ${ }^{2}$ Whereas they treat liquidity as the price sensitivity in response to recent trade in the given asset, we estimate the price sensitivity from the large cross-section of assets. What these price sensitivities represent, and what should be expected for the cross-section of returns, is well understood from the perspective of informational asymmetry. As informational asymmetry increase in the market both illiquidity, as measured by Kyles' lambda, and expected returns increase (Vayanos and Wang, 2011). ${ }^{3}$ Hence, our interest lies in the relative degree of price sensitivity in an asset, and what the aggregates indicate for the market. The measures we rely on for this coincide with the network concept of connectedness, and can be made to closely proxy the network diameter. The empirical challenge of these measures stems from the large number of assets traded on the market. Our solution is to

\footnotetext{
${ }^{2}$ Campbell, Grossman, and Wang (1993) shows that these types of dynamic relations are to be expected. But, limiting the focus to the univariate case restricts the estimates to capture price reversions.

${ }^{3}$ In addition to the financial literature on asymmetric information and market frictions, there is also a related strain of literature about the informativeness of trade (Hasbrouck, 1991a,b).
} 
express the network through a large number of regularized regression, akin to a type of graphical neighborhood selection (Meinshausen and Bühlmann, 2006).

The challenge that follows from the immense number of assets has in previous studies been handled through dimension reduction through statistical commonalities. Hasbrouck and Seppi (2001) study the co-variation of the commonalities in returns and order flows. With the common factors of returns and order flows they reduce the dimensions of the empirical study, thus making traditional tools available to measure sensitivities. Korajczyk and Sadka (2008) expands this approach to describe the commonality of liquidity using eight liquidity measures. Hallin, Mathias, Pirotte, and Veredas (2011) further extends this approach by exploiting dynamics in the commonalities. Both of these studies also finds that their measures of market liquidity indeed help explain cross-sectional variations in returns. We depart from this literature through the manner in which we handle the high-dimensionality of the markets. Instead of reducing the dimensions using commonalities we make use of regularized regression equations. This approach eliminates standard statistical issues existing in the factor model literature such as the calculation of the number of factors or imposing identification restrictions, see Bai and $\mathrm{Ng}$ (2002b, 2013). Empirically, our departure from the factor model literature enables us to analyze pricing effects with greater flexibility since it removes the asset-specific restriction that relevant sensitivities solely stem from market commonalities.

We regularize estimates of the price-trade relations across many assets jointly to form a network to describe the cross-asset relations. Network representation are popular for many applications where it is crucial to understand the rate of transmission or flow within a set of objects, including but not exclusive to: logistics, communications, medicine and contagion. In similar fashion to these applications we want to know to what rate shocks permeate the network. Here, what degree of price adjustments is an asset subject to when trade occur within the network. To measure this we decompose the network of $N$ assets into neighborhoods which may or may not be overlapping. Neighborhoods are unknown and thus brings two challenges; neighbor selection and sensitivity estimation. This type of statistical challenge is common and has been given much attention since Tibshirani (1996). Neighborhood selection for an asset implies that we seek to separate the noise trading from those trades that deliver news about the future cash flows for the given asset. The second part implies estimating the degree of price sensitivity in an asset in response to trade adjustments among its neighbors. To accommodate 
these two issues we make use of an elastic net estimator. This estimator is a convex combination of first and second norm regularization on the parameter vector of a linear regression framework. Thus, it nests the common least absolute shrinkage and selection operator (lasso) and ridge estimators.

We estimate trade networks over 52 years of daily data starting 1965. Returns and order flows are collected from CRSP. Each month we estimate the network using the assets that are fully available that month. Thus we can compute monthly estimates of the distribution of price sensitivities (asset connectedness) across assets. We find that asset-level connectedness is a priced source of risk. The return premium of classical no-cost long-short portfolios based on connectedness, in excess to the five Fama-French factors, are statistically significant at all conventional levels. Since the magnitude of the average daily order flows have greatly increased during the last 20 year we also split the sample into the periods before and after 1995. We find that while the magnitude of order flows have changed their economic function appears to have stayed the same. Both in the before and after periods we find that asset connectivity to the trade network is a priced risk. The periods only differs by the size of the return premium, before 1995 the average monthly return premium was 0.39 percent, in the later period this increased to 0.77 percent.

In addition, we find that the network diameter of the market is closely approximated through the cross-sectional average of the asset connectedness. The diameter describe the longest distance within a network and in our case conveniently characterize the market level of illiquidity. As the diameter changes over time we find that it tend to co-vary strongly with financial distress. The financial crisis of 2008, the end of the dot-com bubble and 'Black Monday' in 1987, are all events that coincide with very low market diameter. Treating news in the market diameter as a macro-factor we create sorts based on the assets absolute return sensitivity. These portfolio sorts are found to have modest, but significant, return premium in excess to the five Fama-French factors.

The rest of the chapter is outlined as follows. In Section 4.2 we define the networks along with the estimators and connectivity measures. The development of the network features over time and asset pricing results are reported in Sections 4.3 and 4.4. Section 4.5 concluded and summarizes our findings. 


\subsection{A network of trade-price relations}

In this section we present how the role of order flows in transmitting news translates into a network representation. Effectively disseminating large flows of information onto financial markets is an important factor to make the markets liquid. Trade and order flows in asset pricing and price discovery is an extensive field of research, for our framework we focus on pricing impacts going back to Kyle (1985). Caballe and Krishnan (1994) provides an extension to the general equilibrium under asymmetric information in a multi-asset framework. Importantly, in this framework investors learn from observing order flows, which may hold information about future payoffs. ${ }^{4}$ Asset illiquidity, measured through the price sensitivity in response to trade, increase with the degree of informational asymmetry (Vayanos and Wang, 2011). Our network connections represent these sensitivities, hence assets that are highly connected are less liquid. In aggregate, large pricing sensitivity of the market implies that informational asymmetry is greater and markets are less liquid. Empirically we observe a large number of trades not knowing which are informed. Uninformed trade does not result in permanent price changes, thus including the pricing sensitivities of these uninformed trades amounts to overfitting. We regularize the estimation to avoid overfitting, while simultaneously accounting for co-movements among returns. Using these networks we construct measures that intuitive relate to the degree of pricing sensitivity on both asset and market level. We update the estimates on a monthly basis, thus allowing the networks to evolve over time and enabling time varying asset and market liquidity.

Our interest is in the price adjustments in response to changes in order flows. Highly sensitive assets are less liquid, which allows investors to demand a premium. Empirically, there are many measures of liquidity that could form the basis of our network representation. One prominent measure was proposed by Pastor and Stambaugh (2003). They estimate price sensitivities and construct market level metrics on the basis of firm specific order flows. Denote the return of asset $(i)$ in $\operatorname{month}(t)$ and day $(d)$ as $r_{i, t, d}$, and let $\tilde{r}_{i, t, d}$ be the excess return relative to the market. We denote the re-scaled million dollar volume of trades $\nu_{i, t, d} \cdot{ }^{5}$ Order

\footnotetext{
${ }^{4}$ Additional, prominent papers that explore the multi-asset perspective and liquidity spillovers include Chowdhry and Nanda (1991), Subrahmanyam (1991) and Cespa and Foucault (2014).

${ }^{5}$ The re-scaling is simply through the monthly standard deviation of the order flows. This is a departure from the framework outlined in Pastor and Stambaugh (2003). They later control for the level of the estimate when forming their measure. We deviate from this to make the approach more compact.
} 
flows signed by the excess returns is denoted $\tilde{\nu}_{i, t, d-1}$,

$$
\tilde{r}_{i, t, d}=a_{i, t}+\phi_{i, t} r_{i, t, d-1}+\gamma_{i, t} \tilde{\nu}_{i, t, d-1}+u_{i, t, d}
$$

The $u_{i, t, d}$ and $a_{i, t}$ are idiosyncratic errors and monthly intercepts respectively. The lagged return is also included in the model as a general control for omitted factors. The parameter of interest is the $\gamma_{i, t}$ of the regression equation. This parameter is estimated on a monthly basis asset by asset. The cross-sectional average sensitivity, $N^{-1} \sum_{i=1}^{N} \gamma_{i, t}$, is the basis of the market liquidity measure Pastor and Stambaugh (2003) propose. This market liquidity is in turn shown to provide a risk premium (Pastor and Stambaugh, 2003; Acharya and Pedersen, 2005). This measure of liquidity targets the effect of price reversions. However, when information is asymmetric trade can also partially reveal private signals, thus introducing effects from the contemporaneous cross-section of order flows on prices. Since the cross-section of assets is very large, extending the framework to include these effects from the cross-section incur a fast increase of the dimensionality of the regression (4.1). Quantifying these price sensitivities is the main purpose of our network representation.

\subsubsection{Estimating asset neighborhoods}

The network representation we shall introduce describes financial markets through the perspective of cross asset price sensitivities. Our network representation is in line with Diebold and Yilmaz (2014) who fit a network based on how accurately returns of a specific asset are predicted by other asset returns. We deviate from Diebold and Yllmaz (2014) by defining neighborhoods of assets as the absolute value of the return sensitivity to order flows, instead of other asset returns, as indicated in Equation 4.1. This sensitivity measure, in return, defines the cross asset sensitivity to order flows. ${ }^{6}$

To clarify our framework, formulate the network as $G=\{V, E: \lambda\}$. The nodes, denoted $V$, represent the financial assets. The edges of the network, $E$, denotes cross asset relations, and the impact of order flows are $\lambda: E \rightarrow \mathbb{R}^{+}$. The nodes and edges of a network consisting of $N$ assets can be fully characterized by an $(N \times N)$-matrix, which we shall denote $\Gamma$. To form a network representation

\footnotetext{
${ }^{6}$ Full estimation of the dynamics that order flow induce on returns and future trade is beyond the scope of our estimation. We argue however that a summary of the initial impacts of trade is sufficient to quantify the level of informational asymmetry in the market.
} 
of short term order flow impacts we let the weights be the price sensitivities to changes in incident asset order flows. This model makes our network directional, which means that an edge from asset $i$ to $j$ does not imply a reciprocal edge from $j$ to $i$. All assets that hold edges to a common asset $i$ are considered part of the neighborhood of $i$. What assets that are contained in a neighborhood is unknown and there are many candidates. Hence, this provides a form of model selection problem. This high-dimensional neighborhood selection becomes explicit as we extend the model of Pastor and Stambaugh (2003) for a asset $i$ to include the full cross-section of order flows.

$$
\tilde{r}_{i, t, d}=a_{i, t}+\phi_{i, t} r_{i, t, d-1}+\gamma_{i, t}^{(i)} \tilde{\nu}_{i, t, d-1}+\sum_{j \neq i} \gamma_{j, t}^{(i)} \tilde{\nu}_{j, t, d-1}+u_{i, t, d} \quad d=1 \ldots D
$$

In equation (4.2), the number of cross asset sensitivities $\gamma_{j, t}^{(i)}$ can be far more numerous than the observations available if $T$ is small. In order to estimate these parameters we rely on methods of regularization, namely neighborhood selection introduced by Meinshausen and Bühlmann (2006). In this approach, the network estimation is performed by estimating each asset neighborhood independently. Thus, treating the network as a collection of $N$ neighborhoods, the full network estimated using $N$ linear models. The independent estimation of each one of the $N$ equations imply a directed network, where the direction is from the right-handside to the left-hand-side of (4.2) for $N$ equations. ${ }^{7}$ This structure lends itself in an intuitive manner to the idea of predictive regressions. The estimation of $N$ equations and neighborhoods implies that the independently estimated neighborhoods can overlap, and assets with a common neighbor are not necessarily themselves neighbors.

There is a growing literature of financial studies that makes use of regularized methods for model selection and return prediction, among some of the recent are Chinco, Clark-Joseph, and Ye (2017), Kozak, Nagel, and Santosh (2017) and Feng, Giglio, and Xiu (2017). We follow along a similar procedure of parameter regularization as in these studies, thus estimating the high-dimensional model (4.2) while penalizing the size and impact of the asset neighborhoods.

There are several methods that can be used for the above-mentioned neighborhood regularization problem. Among the typical types of regularization is the lasso estimator (Tibshirani, 1996), which penalizes the absolute values of the parameter

\footnotetext{
${ }^{7}$ Estimating the network relations in a simultaneous manner can increase efficiency, but would restrict the ways neighborhood sparsity could differ between assets.
} 
vector. This estimator performs simultaneous model selection and parameter estimation. This form of regularization is proposed by Meinshausen and Bühlmann (2006) for the neighborhood estimation. An attractive feature of the lasso estimator is its ability to impose sparsity through soft thresholding. Under standard regularity conditions on the error terms along with an assumption of sparsity, few relevant regressors in the linear form of the model, the estimator maintains model selection consistency (Zhao and Yu, 2006). Put differently, this regularization implies that only trade in a small number of assets should be regarded as informed for each given asset. This often attractive assumption is not strictly in line with our economic framework since informed traders do not necessarily maintain and trade small portfolios. Instead, it is possible that there are a large number of private signals that are active simultaneously. Hence, there may be many relevant predictive order flows that may also follow a group structure. And thus, a strict sparsity assumption may therefore not be suitable.

An alternative to the lasso type regularization is the penalization of parameter estimates under the second norm, which amounts to ridge estimation. This estimator does not promote sparsity in the manner of the lasso but still attenuates the parameter estimates. This form of regularization implies the different economic extreme where trade in all assets are economically relevant for all assets.

We conjecture that the optimal choice for our application lies between these two extremes of the lasso and ridge estimators. Thus, we propose to adopt a more general form of regularization, estimating the parameters of (4.2) as,

$$
\begin{gathered}
\left(\hat{\gamma}_{., t}^{(i)}\right)=\operatorname{argmin}\left\|\tilde{r}_{i, t, d}-a_{i, t}-\phi_{i, t} r_{i, t, d-1}-\gamma_{i, t}^{(i)} \tilde{\nu}_{i, t, d-1}-\sum_{j \neq i} \gamma_{j, t}^{(i)} \tilde{\nu}_{j, t, d-1}\right\|_{2} \\
+\lambda \sum_{j \neq i}\left(\alpha\left\|\gamma_{j, t}^{(i)}\right\|_{1}+(1-\alpha)\left\|\gamma_{j, t}^{(i)}\right\|_{2}\right)
\end{gathered}
$$

known as the elastic net proposed of Zou and Hastie (2005). In (4.3), the value of parameter $\alpha \in[0,1]$ leads to different degrees and forms of regularization. With $\alpha=1$ the penalization is on the absolute parameter vector, which corresponds to the lasso estimator. Alternatively, $\alpha=0$ moves the penalization onto the second norm of the parameter vector, which provides the ridge estimator. This flexibility allows the form of regularization to vary between assets, some of which may be well suited to the lasso type sparsity assumptions while others are better accommodated by the ridge estimator. 
Denoting estimates of the $i$ th neighborhood $\hat{\Gamma}^{(i)}=\left\{\hat{\gamma}_{j} ; j=1 \ldots N\right\}$ we construct the network as,

$$
\widehat{\boldsymbol{\Gamma}}=\left(\begin{array}{llll}
\hat{\Gamma}^{(1)} & \hat{\Gamma}^{(2)} & \ldots & \hat{\Gamma}^{(N)}
\end{array}\right)=\left(\begin{array}{cccc}
\hat{\gamma}_{1}^{(1)} & \hat{\gamma}_{1}^{(2)} & \ldots & \hat{\gamma}_{1}^{(N)} \\
\hat{\gamma}_{2}^{(1)} & \hat{\gamma}_{2}^{(2)} & \ldots & \hat{\gamma}_{2}^{(N)} \\
\vdots & \vdots & \ddots & \vdots \\
\hat{\gamma}_{N}^{(1)} & \hat{\gamma}_{N}^{(2)} & \ldots & \hat{\gamma}_{N}^{(N)}
\end{array}\right)
$$

where the non-zero entries of the $i$ th column are said to be in the neighborhood of the $i$ th asset. Notice that defining market liquidity as the statistic $N^{-1} \operatorname{tr}(\widehat{\boldsymbol{\Gamma}})$ provides a close analogue to the basis for the market liquidity measure of Pastor and Stambaugh (2003).

\subsubsection{Calibrating the regularization}

The estimator defined in (4.3) admits several standard methods of regularization. The properties of different types of regularization differ widely and the choice of which to use is largely determined by the sparsity assumption. If the parameter $\alpha$ is set to zero the estimator no longer imposes sparsity in the neighborhoods. The resulting ridge estimator shrinks the parameter vector in a manner that attenuates the point estimates, but does not set any parameters exactly equal to zero. Hence, for the financial interpretation this implies that all trade is in part informative.

Implementing this estimator we let $\alpha$ vary over a grid within the interval of zero to one. In this way we can account for group structures and a large number of relevant predictors, while still retaining the possibility of setting point estimates exactly equal to zero. There are alternative methods for how to choose the parameter $\alpha$ and the level of regularization $\lambda$. Typical approaches are either by using cross-validation or an information criterion. For our purpose, due to computational constraints, we will rely on an information criterion to calibrate the regularization.

We vary the parameter $\alpha$ and the level of regularization along two grids. The grid for $\alpha$ is formed by twenty values in the interval 0 and 1 . For the level of regularization is we use a grid of 100 values on a linear path in log-scale. For every 
given value we compute the degrees of freedom following Zou and Hastie (2005),

$$
\hat{d f}=\operatorname{tr}\left(X\left(X^{\prime} X+\lambda \boldsymbol{I}\right)^{-1} X^{\prime}\right)
$$

Here $X$ denotes the set of active predictors given a level of regularization and $\alpha$. With these estimates of the degrees of freedom we find the parameter combination that minimizes the Schwartz information criterion.

Both parameters $\alpha$ and $\lambda$ are calibrated on each asset independently. This asset level calibration allows for very different neighborhoods across assets on the financial markets. This flexibility makes it possible for some assets to be connected to a very large number of neighbors, while others only have small neighborhoods.

By the union of the neighborhoods we form a full network representation of the financial assets. In the following section we reconnect this network to our previous economic framework by introducing measures of network connectivity and centrality that intuitively link our representation to the economic issues of illiquidity.

\subsubsection{Asset and market connectedness}

Assets that are characterized by high levels of price sensitivity in response to order flows are, in the economic framework previously outlined, less liquid. In our approach these are the assets that are highly connected to our network. In order to summarize the connectedness of individual assets and the larger characteristics of the network we rely on measures that are common in the network literature. ${ }^{8}$ Many of these measures, such as the degree, are also found in previous financial research, for example Diebold and Ylmaz (2014). Our networks are formed on the price sensitivities in response to trade.

The order flow network that was introduced in the previous section describes the behavior of asset returns in response to trade. Our basis for the network connectivity is through the volume and size of these cross asset connections. To measure the asset level of connectivity we compute the asset degree. The degree, denoted $d_{i}$, is the number of edges associated with a given node, weighted by respective price sensitivities. ${ }^{9}$ Diebold and Yllmaz (2014) refers to this type of

\footnotetext{
${ }^{8}$ Some of these are handled in a general overview in Borgatti and Everett (2006)

${ }^{9}$ Since network edges are not reciprocal the degree of a node can be separated into the in-degree and the out-degree. However, for our purpose we restrict our attention to the in-degree, which we simply refer to as the degree. The out-degree shifts the parameter interpretation towards a form of trade informativeness.
} 
connectedness measure as directional connectedness.

$$
d_{i, t}=\sum_{\substack{j=1 \\ j \neq i}}^{N}\left|\gamma_{j, t}^{(i)}\right| .
$$

The degree distribution across assets is the first source of market level measures. Basic properties such as central tendencies and variance carry interesting implications for the market sensitivity to order flows. For example, an increasing average degree may imply that the markets overall are becoming more sensitive to order flows, which is indicative of higher levels of informational asymmetry.

Similar to Pastor and Stambaugh (2003) we aggregate the asset characteristics to gain a perspective of the market. It turns out the cross-sectional average of the asset degree is closely related to the network concept of diameter, which is the longest distance between any two nodes in the network. In very large networks this exact computation of the estimated diameter is computationally unfeasible. Erdös and Rényi (1959) propose an approximation to the network diameter, which is $D=\ln (N) / \ln \left(\mathrm{E}\left(d_{i}\right)\right)$. Since the number of stocks is very large and increases slowly over time, the main source of variation in the diameter stems from the denominator. Hence, for our purposes we will use the cross-sectional average of the asset degree as a proxy for the market connectedness and diameter.

As price sensitivities $\gamma_{j}$ vary over time we find varying levels of asset connectedness reflecting the individual stocks level of liquidity. Low asset degree implies that the asset is robust against changes in order flows in other assets on the market. In turn this indicates a high level of liquidity. Extending this reasoning to the market diameter a large market diameter characterize a liquid market where

the effects of order flows permeate the network slowly. We will use these two characteristics, asset and market connectedness, to approach the level of asset and market illiquidity. As the measures adjust to reflect the changing circumstances on the markets we expect to find that there is a return premium for investors that are exposed liquidity risk as measured by our characteristics.

\subsection{Network features and characteristics over time}

In this section we describe the basic features of the estimated trade network and assess its development since 1965. The main interest for our analysis are the series of network measures such as asset and market connectedness. We are especially 
interested in whether the development of the network features is in line with the expectation for measures of financial liquidity. For this purpose, we evaluate the network features over time, and compare these features to known periods of financial distress we find that extremes in these market and asset characteristics.

We make use of daily financial data from CRSP, we use all stocks recorded with share code 10 or 11 . For our purposes we exclude all stocks with prices below $\$ 5$ or over $\$ 1000$, as well as stocks with a market capitalization below the first quartile of the capitalization distribution of the NYSE. We consider the time period of 1965 until 2018, which in the end provides an unbalanced panel of more than 10000 assets. As we implement our estimation monthly we drop assets that are partially missing during the month in question. The number of daily returns available varies between around 1000 to around 3000 .

The neighborhood of each asset is projected using an elastic net, which selects the most relevant assets pertaining to pricing sensitivities to order flows. Repeating this estimation for all assets in every month we form a network independently each month. Every time the network is estimated we compute the network connectedness as well as the distribution of asset connectedness. The connectedness is measured by asset degree (4.6), along with its cross-sectional average.

Figures 4.1 and 4.2 display the development of the cross-sectional average of order flows over time. Obvious from the pictures is that the variance of order flows is greatly increasing. However, the price sensitivity estimates will remain comparable over time since we re-scale the variance of order flows in each monthly estimation.

Figure 4.1: Order flows from 1965 to 2018

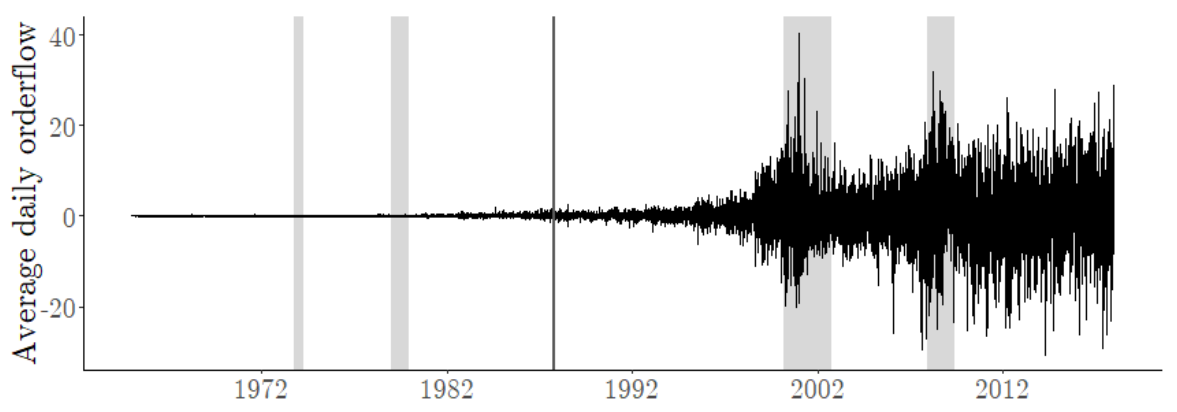

The average signed daily order flows in millions of dollars from 1965 to 2018 . Shaded areas correspond to the first and second oil crisis, 'Black Monday' of 1987, the Dot-com bubble and the Great recession. 
Furthermore, Figure 4.1 reveals that the largest shift in order flow magnitudes starts during the mid-90s. ${ }^{10}$ Figure 4.2 separates the order flow into 4 sub-periods: pre-1980, 1980 to 1995, 1995 to 2005, and post-2005. Within each of the sub period we find that the order flows to tend to react to some periods of financial distress, but this reaction is relatively mild with small increases in the variance of order flows. Figures 4.1 and 4.2 display the timing of the two oil crises, 'Black Monday' of 1987, the dot-com bubble and the 'Great recession'. In particular, we find that the oil crisis did not have a large affect the order flows. However, the remaining events of the 'Black Monday', the 'dot-com' bubble and the 'Great Recession' do seem to coincide with periods of increased order flow volatility.

Next we assess the asset level connectedness to the network, which is computed using the respective asset degree. This measure accounts for the number of assets in a neighborhood, weighted by the pricing sensitivities to the corresponding order flows. The three panels of Figure 4.3 show the mean number of neighbors (middle panel), along with the 5th and 95th percentiles (bottom and top panels, respectively). The mean neighborhood size displays a weak positive trend from 1980 until 2000, and is relatively stable during the years preceding and following this period. The lower panel of Figure 4.3 displays that the 5th percentile of the neighborhood size distribution is very stable around 20 assets over the whole period. Contrary to the findings on the mean and 5th percentile of the distribution, we find that the upper end of the distribution is much less stable. The number of assets included in the 95th largest neighborhoods vary from below 100 in 1965 to almost 175 in 1999. This reflects the same positive trend found in the average neighborhoods from 1980 until 2000, but the size of the change over time is more pronounced.

Assigning the price sensitivities to the order flows of the respective neighborhoods we form our degree measure of connectedness. In Figure 4.4 the degree of the assets reveals variation over time that coincides with the periods of financial turbulence.

We do not find the same trending behavior as the neighborhood size revealed. This implies that while assets became connected to an increasing number of neighbors, their average return sensitivity decreased, thus keeping the level of connectedness stable. In the same way that market diameter dropped during 1987, late 1990s' and following 2008, we find that the whole distribution of connectedness

\footnotetext{
${ }^{10}$ This introduces an additional question; Does the role of order flows change at the time of the great shift in its variance? In Appendix 4.B we split the sample to evaluate the our network before and after 1995 .
} 
Figure 4.2: Average order flows 1965 to 2018
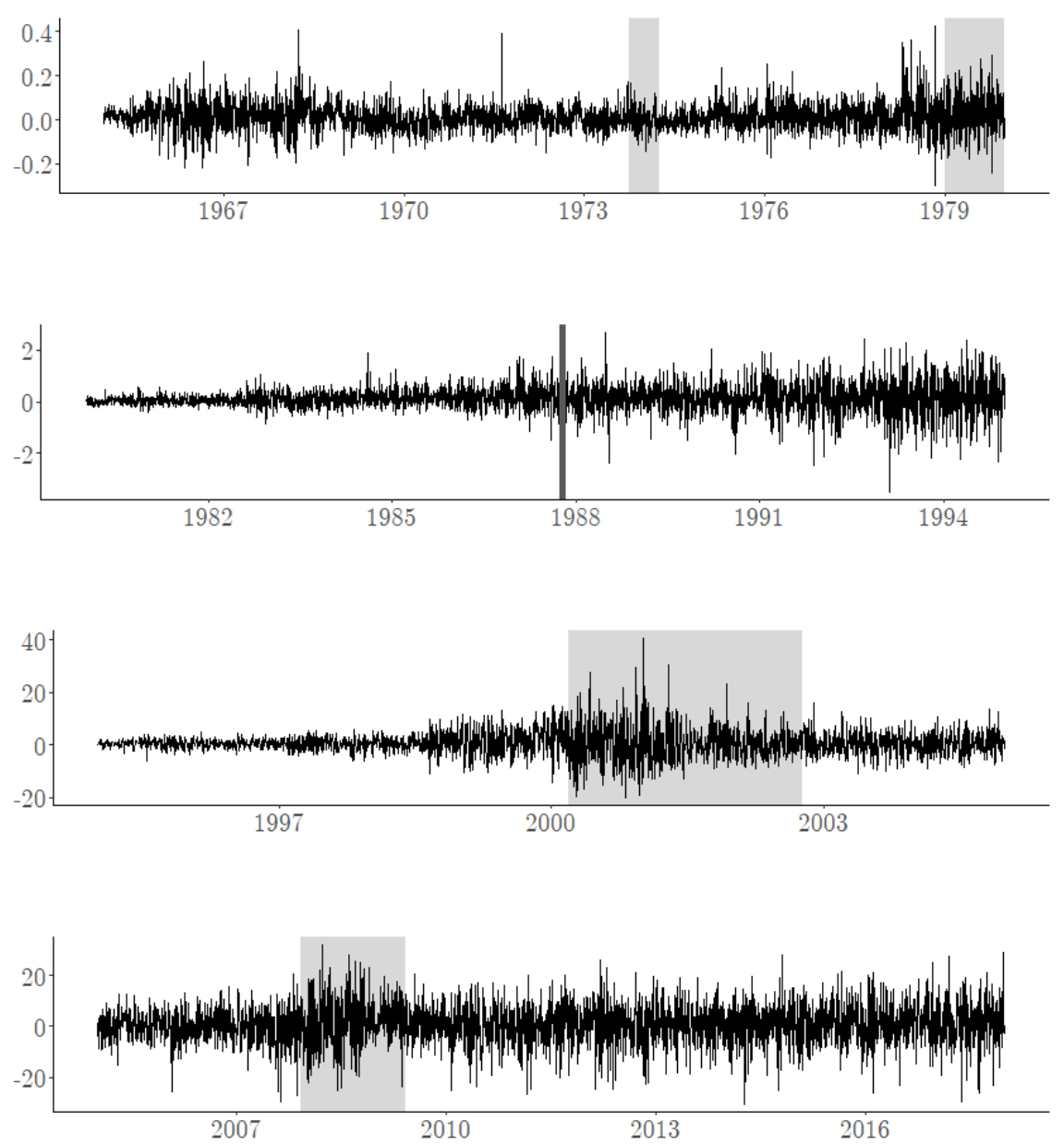

Average signed daily order flows in millions of dollars from 1965 to 2018 . Order flows are divided into four sub-periods, pre-1980, 1980 to 1995, 1995 to 2005, and post-2005. Shaded areas correspond to the first and second oil crisis, 'Black Monday' of 1987, the 'dot-com' bubble and the 'Great recession'. 
Figure 4.3: Realized neighborhood sparsity 1965 to 2018
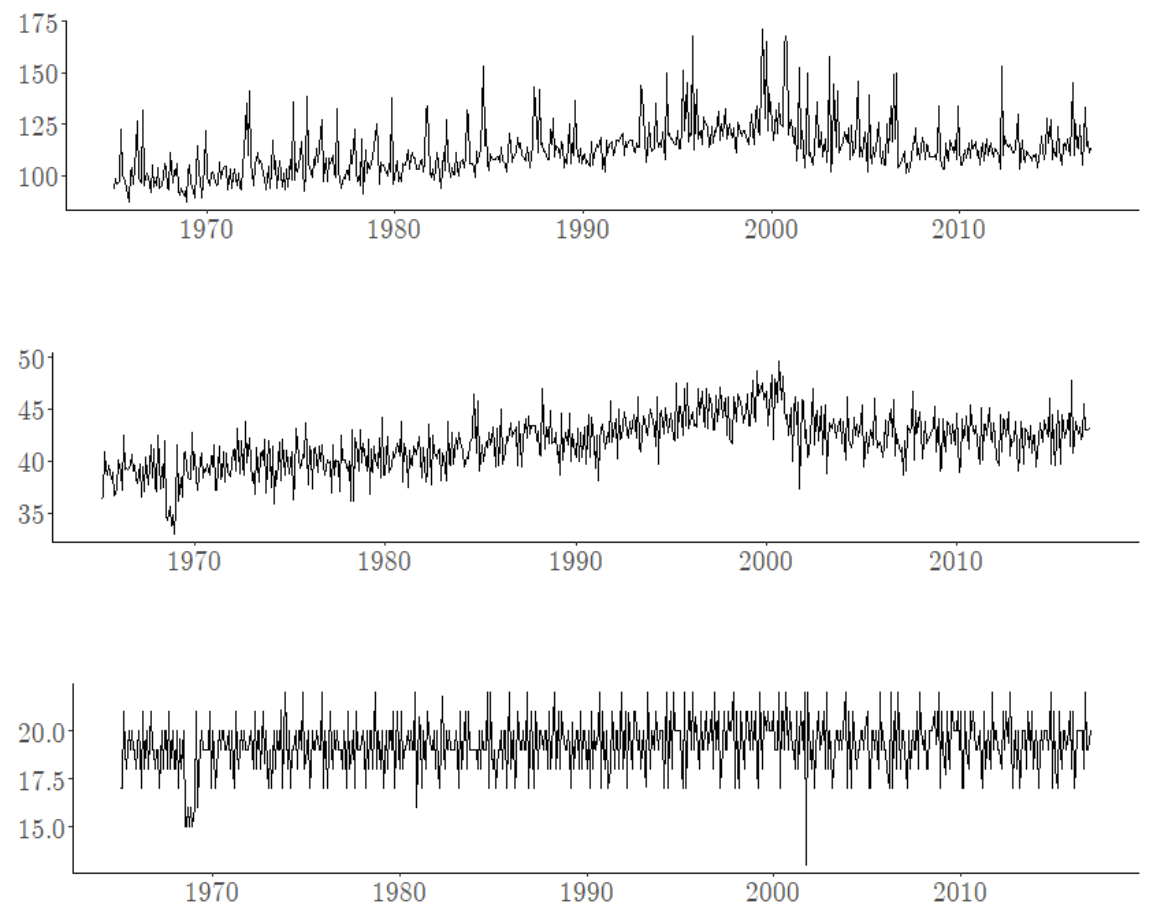

Number of assets in neighborhood, mean(middle panel), 5th(bottom panel) and $95 \mathrm{th}$ (top panel) percentile of asset in-degree.

shifts upwards during these periods. Hence, the drop in network degree is not solely driven by outliers among the assets but rather an increase in connectedness across the whole market.

Figure 4.5 shows the development of the variance in connectedness over time. This measure is more reflective of extreme events compared to previous descriptive statistics, and it indicates the variation in the connectedness measure. The figure reveals that the deviation from the average connectedness greatly increases just before the early 2000s and during the financial crisis starting 2008. The crisis of 1987 is also associated with larger deviations across assets, but not nearly resembling the levels of the following years. In other words, latter periods of financial turbulence were generally affiliated with higher levels of asset heterogeneity with respect to informational asymmetry compared to earlier periods. 
Figure 4.4: Network degree

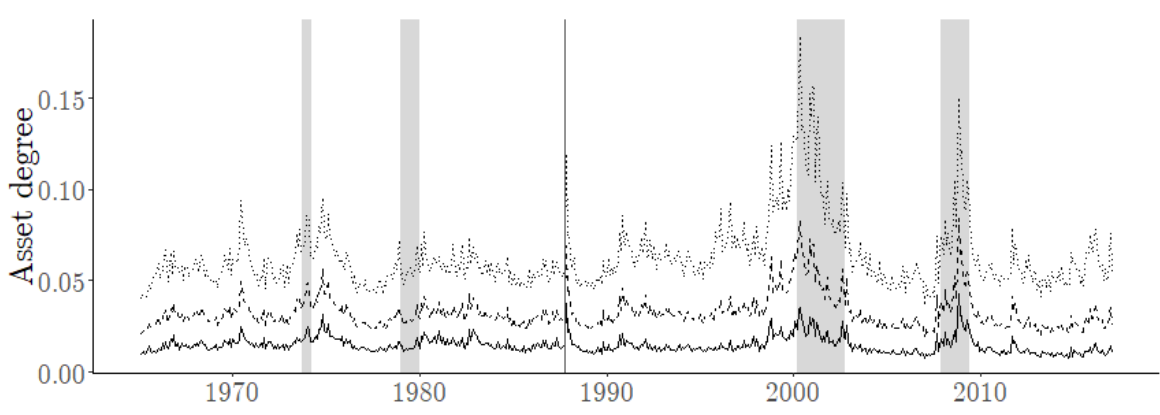

Monthly average(dashed line), 5th(solid line) and 95th(dotted line) percentile of asset in-degree. Shaded areas correspond to the first and second oil crisis, 'Black Monday' of 1987, the Dot-com bubble and the Great recession.

The reported market connectedness is used to approximate variations in the market diameter, which we evaluate as a source of risk on financial markets. We report the extent to which these innovations in the market connectedness correlate with the five Fama-French factors, which are often found to have relatively large explanatory power for the expected returns. The pairwise correlation between the respective factors and the innovations in the market diameter is reported in Table 4.1.

Table 4.1: Diameter innovations - Correlation matrix

\begin{tabular}{lccllll}
\hline Diameter & 1 & & & & \\
Mkt & -0.02 & 1 & & & \\
SMB & $0.10^{* *}$ & $0.28^{* * *}$ & 1 & & & \\
HML & 0.01 & $-0.26^{* * *}$ & $-0.09^{* *}$ & 1 & & \\
RMW & -0.04 & $-0.24^{* * *}$ & $-0.35^{* * *}$ & $0.08^{* *}$ & 1 & \\
CMA & -0.01 & $-0.39^{* *}$ & $-0.11^{* * *}$ & $0.69^{* * *}$ & -0.03 & 1 \\
\hline Pearson correlation coefficient. (***) $\mathrm{p}<0.01,\left({ }^{* *}\right) \mathrm{p}<0.05,\left({ }^{*}\right) \mathrm{p}<0.1$. \\
Based on 623 months of consecutive returns, 1965 until 2017.
\end{tabular}

We find a significant positive correlation between diameter innovations and the size factor (SMB), indicating that positive news in the diameter tend to be accompanied by higher returns in the portfolio of small stocks. The pairwise correlations between the diameter innovations and the market, HML and CMA, respectively are not significantly different from zero. Thus, the diameter innovations are distinct from the Fama-French factors except for the size factor. This finding is in line 
Figure 4.5: Asset degree standard deviation

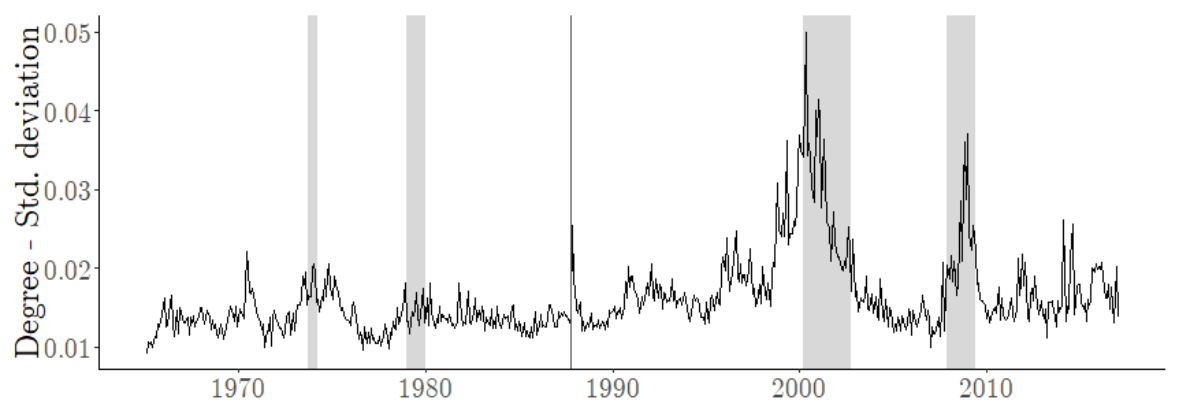

Standard deviation of asset in-degree from 1965 to 2018. Shaded areas correspond to the first and second oil crisis, 'Black Monday' of 1987, the Dot-com bubble and the Great recession.

with the expectation that illiquidity, and asymmetric information, is particularly linked to assets of low market capitalization.

In sum, the network connectedness feature we have emphasized is meant to quantify the degree of illiquidity of an asset. We find that the average connectedness of the assets appear to co-vary with periods of general illiquidity and financial distress. Based on these results, we next assess whether the previously proposed measures of asset connectedness and network diameter capture risk that is priced in the cross-section of assets.

\subsection{Is asset and market connectedness priced}

If the average connectedness of the market and the level of asset connectedness successfully measure a facet of illiquidity, we expect these connectedness measures to predict differences in expected returns. In this section we evaluate if there is a significant return premium to asset connectedness beyond what Fama-French factors can account for. For this purpose, we form decile portfolio sorts based on connectedness and market diameter. We assess the portfolios conditional on the model specification,

$$
r_{i}-r f=\alpha+\beta_{1} M k t+\beta_{2} S M B+\beta_{3} H M L+\beta_{4} R M W+\beta_{5} C M A+\varepsilon_{i},
$$

where $r_{i}$ denotes the return on the portfolio sort and $r f$ is the risk-free rate. A statistically significant return premium, $\alpha$, after conditioning on the Fama-French 
factors, implies that the risk captured by our measures is not explained by the Fama-French factors.

\subsubsection{Asset connectedness}

We first assess the asset connectedness by forming decile portfolio sorts. To avoid overemphasizing small stocks and contaminating the return premium we form all decile sorts such that the market capitalization is similar across the portfolios. Fir this purpose, we define market capitalization as the asset price times the number of outstanding shares. An asset $i$ is assigned to a decile sort in month $t$ based on the relative level of connectedness in month $t-1$. All assets with an estimated degree of connectedness is then assigned to a portfolio sort and all assets within each sort is given an equal weight. The portfolio sorts on asset connectedness is presented in Table 4.1.

Table 4.1: Decile portfolio sorts based on asset connectedness

\begin{tabular}{lcccccccccc}
\hline \hline & $(1)$ & $(2)$ & $(3)$ & $(4)$ & $(5)$ & $(6)$ & $(7)$ & $(8)$ & $(9)$ & $(10)$ \\
\hline $\mathrm{N}$ & 58 & 66 & 76 & 88 & 100 & 117 & 137 & 170 & 224 & 347 \\
$\mathrm{ME}$ & 609 & 656 & 648 & 649 & 647 & 644 & 649 & 652 & 649 & 654 \\
\hline $\bar{r}$ & 0.59 & 0.64 & 0.73 & 0.79 & 0.81 & 0.90 & 0.94 & 0.99 & 1.12 & 1.10 \\
$\alpha$ & -0.03 & -0.01 & 0.06 & 0.11 & 0.09 & 0.20 & 0.22 & 0.29 & 0.52 & 0.66 \\
& $(.07)$ & $(.06)$ & $(.06)$ & $(.06)$ & $(.05)$ & $(.05)$ & $(.05)$ & $(.04)$ & $(.06)$ & $(.08)$ \\
\hline \multirow{2}{*}{$M k t$} & 0.84 & 0.86 & 0.89 & 0.93 & 0.96 & 0.98 & 1.02 & 1.05 & 1.08 & 1.19 \\
& $(.03)$ & $(.02)$ & $(.02)$ & $(.02)$ & $(.02)$ & $(.02)$ & $(.02)$ & $(.01)$ & $(.02)$ & $(.02)$ \\
$S M B$ & -0.00 & 0.06 & 0.13 & 0.14 & 0.19 & 0.26 & 0.32 & 0.43 & 0.55 & 0.75 \\
& $(.03)$ & $(.03)$ & $(.02)$ & $(.02)$ & $(.02)$ & $(.02)$ & $(.02)$ & $(.02)$ & $(.03)$ & $(.03)$ \\
$H M L$ & 0.18 & 0.21 & 0.23 & 0.22 & 0.20 & 0.15 & 0.12 & 0.05 & -0.09 & -0.26 \\
& $(.04)$ & $(.04)$ & $(.03)$ & $(.04)$ & $(.04)$ & $(.03)$ & $(.03)$ & $(.03)$ & $(.04)$ & $(.05)$ \\
$R M W$ & 0.28 & 0.28 & 0.28 & 0.26 & 0.25 & 0.22 & 0.17 & 0.06 & -0.17 & -0.61 \\
& $(.04)$ & $(.03)$ & $(.03)$ & $(.03)$ & $(.03)$ & $(.03)$ & $(.03)$ & $(.03)$ & $(.07)$ & $(.05)$ \\
$C M A$ & 0.25 & 0.18 & 0.16 & 0.13 & 0.14 & 0.13 & 0.14 & 0.07 & -0.01 & -0.34 \\
& $(.06)$ & $(.05)$ & $(.04)$ & $(.04)$ & $(.05)$ & $(.05)$ & $(.04)$ & $(.04)$ & $(.06)$ & $(.06)$ \\
\hline$R^{2}$ & 0.78 & 0.86 & 0.89 & 0.91 & 0.92 & 0.93 & 0.94 & 0.96 & 0.96 & 0.95 \\
\hline
\end{tabular}

Intercepts $(\alpha)$ are multiplied by 100, standard errors are reported in parentheses. The raw returns are denoted $\bar{r}$ and are multiplied by 100. Market capitalization (ME) is the number of outstanding shared multiplied by the price, reported in millions of dollars. Estimates are based on 623 consecutive months of data. Standard errors are robust to heteroskedasticity.

The sorts contain a varying number of assets. The lower decile sorts contain fewer assets that the higher decile sorts based on asset degree. Hence, the assets 
with very high market capitalization tend to be much less connected in our pricingtrade network. Inversely we find that the assets that are highly connected in our network on average have a much lower level of capitalization. Intuitively this fit the perception that issues of illiquidity is a predominately a small asset characteristic.

The parameters reported in Table 4.1 are estimated under model specification 4.7. Of particular interest is the estimated return intercept $\hat{\alpha}$. We find that the portfolio sorts of assets with low level of asset connectedness generally provide little or no significant return premium. The assets with high connectedness, however, provide a statistically significant premium in excess to what the Fama-French factors can account for.

Next we form a no-cost portfolio that takes a long position in the highly connected assets and a short position in the assets that have the lowest degree in the network. In Table 4.2, we present the estimation results of the regression of these portfolio returns on the five factors. The return premium of connectedness is statistically significant at all conventional significance levels. Hence, we find that the asset connectedness characteristic measures risk that is priced. The intercept estimates are scaled by 100 . Thus, the monthly return premium on the long-short portfolio is approximately $0.69 \%$.

Table 4.2: Long-Short portfolio sorts based on asset connectedness

\begin{tabular}{rcrrrrrrr} 
& $\alpha$ & $M k t$ & $S M B$ & $H M L$ & $R M W$ & $C M A$ & $R^{2}$ & $S R$ \\
\hline$(10)-(1)$ & 0.69 & 0.36 & 0.75 & -0.44 & -0.89 & -0.59 & 0.79 & 0.30 \\
& $(.12)$ & $(.04)$ & $(.05)$ & $(.06)$ & $(.06)$ & $(.09)$ & &
\end{tabular}

Intercepts $(\alpha)$ are multiplied by 100, standard errors are reported in parentheses. Market capitalization (ME) is the number of outstanding shared multiplied by the price, reported in millions of dollars. The Sharpe ratio (SR) is annualized using a $\sqrt{12}$ scaling. Estimates are based on 623 consecutive months of data. Standard errors are robust to heteroskedasticity.

Table 4.2 presents a substantial premium to the proposed connectedness measure. Despite this promising result, the calculated premium can be conflating two separate asset characteristics, size and liquidity. To separate these two phenomena and evaluate whether our liquidity premium is robust to the size effect we next analyze new portfolio sorts along both dimensions. 


\subsubsection{Dissecting connectedness using Size and Degree}

The inverse relation between market capitalization and returns is well known in the financial literature. ${ }^{11}$ In the previous results we found assets with high network connectivity are also on average smaller in terms of market capitalization. In this section we disentangle the size premium from the return premium we found among highly connected assets. In order to do this we form two-way conditional sorts. We form quintile sorts of assets based on assets, which have valid estimates of their network degrees. This accounts for the biggest reduction of the available assets since it imposes that the assets must have been traded every day of the last month. In order to ensure a homogeneous level of diversification across portfolios we do not fix the amount of market capitalization in the sorts. Within each size sorted quintile portfolio we rank assets based on their respective connectedness and form additional quintile sorts. Conditional on the five Fama-French factors, model (4.7), we estimate intercepts and exposures for each of the respective 25 portfolios. The results of these estimates are reported in Table 4.3.

The two-way portfolio sorts all hold on average 55 or 56 assets. Market capitalization, however, varies across and within the portfolios. The main differences are within the large portfolio quintile, where the low degree portfolio has a market capitalization of 1322 million dollars while the high degree sort is only of 681 million. Thus, across these degree sorts there may still be lingering effects from assigning large weights to small stocks.

The estimates presented in Table 4.3 display monotonically increasing alpha estimates as the connectedness increase within the quintile of small stocks. The portfolio alpha of small stocks with low connectedness indicate an estimated 0.88 percent monthly return. In the same size sort among highly connected stocks that same return is 1.97 percent. Both of these estimates are statistically significant. This is a key result since it indicates that the return premium we found with respect to asset connectedness is robust to controls for common size effects among small stocks. The same monotonic pattern can be found among the stocks in the forth and fifth size quintiles, but in much smaller magnitudes. The difference between the low and high degree sorts within the fifth size quintile is 0.24 percent. This further the indication that asymmetric information and illiquidity, through pricing impacts, are primarily small stock phenomena.

\footnotetext{
${ }^{11}$ Berk (1995) argues that this relation does not constitute an anomaly.
} 
Table 4.3: Quintile portfolio sorts based on capitalization and asset connectedness

\begin{tabular}{|c|c|c|c|c|c|c|c|c|c|c|}
\hline & Small & $(2)$ & $(3)$ & (4) & Large & Small & $(2)$ & (3) & (4) & Large \\
\hline & \multicolumn{5}{|c|}{ Number of assets } & \multicolumn{5}{|c|}{ Market capitalization } \\
\hline Low & 55 & 55 & 56 & 56 & 56 & 25 & 41 & 72 & 153 & 1322 \\
\hline$(2)$ & 55 & 56 & 56 & 56 & 56 & 25 & 41 & 71 & 152 & 1122 \\
\hline (3) & 55 & 56 & 56 & 56 & 56 & 25 & 41 & 71 & 151 & 1014 \\
\hline (4) & 54 & 56 & 56 & 56 & 56 & 25 & 41 & 71 & 151 & 882 \\
\hline \multirow[t]{2}{*}{ High } & 53 & 55 & 55 & 55 & 55 & 25 & 40 & 69 & 147 & 681 \\
\hline & \multicolumn{5}{|c|}{$\hat{\alpha}$} & \multicolumn{5}{|c|}{$t(\hat{\alpha})$} \\
\hline Low & 0.88 & 0.16 & 0.00 & -0.05 & -0.09 & 13.39 & 2.47 & 0.00 & -0.70 & -1.52 \\
\hline$(2)$ & 1.15 & 0.13 & 0.08 & -0.01 & -0.08 & 16.10 & 1.93 & 1.07 & -0.11 & -1.69 \\
\hline (3) & 1.32 & 0.13 & -0.01 & 0.01 & -0.07 & 16.36 & 1.87 & -0.16 & 0.18 & -1.26 \\
\hline (4) & 1.72 & 0.24 & 0.18 & 0.17 & 0.09 & 17.21 & 3.07 & 2.35 & 2.00 & 1.68 \\
\hline High & \multicolumn{5}{|c|}{$\hat{\beta}_{1}$} & \multicolumn{5}{|c|}{$t\left(\hat{\beta}_{1}\right)$} \\
\hline Low & 0.87 & 0.87 & 0.90 & 0.90 & 0.89 & 48.26 & 48.27 & 46.06 & 42.19 & 52.89 \\
\hline$(2)$ & 0.98 & 1.00 & 0.99 & 0.99 & 0.96 & 44.57 & 48.95 & 47.29 & 43.52 & 69.36 \\
\hline (3) & 1.05 & 1.08 & 1.09 & 1.06 & 1.03 & 40.57 & 50.38 & 54.88 & 58.15 & 62.03 \\
\hline (4) & 1.13 & 1.15 & 1.12 & 1.10 & 1.04 & 36.19 & 50.88 & 48.69 & 49.56 & 65.94 \\
\hline High & \multicolumn{5}{|c|}{$\hat{\beta}_{2}$} & \multicolumn{5}{|c|}{$t\left(\hat{\beta}_{2}\right)$} \\
\hline Low & 0.60 & 0.45 & 0.25 & 0.09 & -0.16 & 24.36 & 16.51 & 8.58 & 3.30 & -7.21 \\
\hline (2) & 0.72 & 0.60 & 0.37 & 0.14 & -0.12 & 23.14 & 20.18 & 11.56 & 4.94 & -6.49 \\
\hline (3) & 0.81 & 0.72 & 0.47 & 0.20 & -0.10 & 23.45 & 23.74 & 15.64 & 7.91 & -4.20 \\
\hline (4) & 0.87 & 0.85 & 0.60 & 0.34 & -0.02 & 19.16 & 23.26 & 18.18 & 9.21 & -0.73 \\
\hline High & 1.02 & 0.98 & 0.65 & 0.45 & 0.11 & 15.36 & 20.45 & 13.91 & 10.23 & 2.35 \\
\hline
\end{tabular}

The quintile sorts along the column are based on market capitalization while sorts along the rows are based on asset degree. Parameters estimated under the model, $r_{i}-r f=\alpha+\beta_{1} M k t+\beta_{2} S M B+\beta_{3} H M L+\beta_{4} R M W+\beta_{5} C M A+\varepsilon_{i}$. Intercepts $(\alpha)$ are multiplied by 100 , standard errors are reported in parenthesis. Market capitalization (ME) is the number of outstanding shared multiplied by the price, reported in millions of dollars. Estimates are based on 623 consecutive months of data. Standard errors are robust to heteroskedasticity. 
Table 4.3: Continued - Quintile portfolio sorts based on capitalization and asset connectedness

\begin{tabular}{|c|c|c|c|c|c|c|c|c|c|c|}
\hline & Small & (2) & (3) & (4) & Large & Small & $(2)$ & (3) & (4) & Large \\
\hline & \multicolumn{5}{|c|}{$\hat{\beta}_{3}$} & \multicolumn{5}{|c|}{$t\left(\hat{\beta}_{3}\right)$} \\
\hline Low & 0.28 & 0.35 & 0.32 & 0.28 & 0.15 & 6.69 & 8.66 & 7.22 & 6.07 & 4.19 \\
\hline$(2)$ & 0.24 & 0.25 & 0.27 & 0.22 & 0.13 & 5.32 & 5.84 & 4.97 & 4.55 & 4.53 \\
\hline (3) & 0.05 & 0.17 & 0.15 & 0.11 & 0.05 & 1.15 & 4.69 & 3.56 & 2.50 & 1.56 \\
\hline (4) & -0.07 & -0.09 & -0.16 & -0.17 & -0.06 & -0.92 & -1.77 & -3.55 & -3.10 & -1.93 \\
\hline High & \multicolumn{5}{|c|}{$\hat{\beta}_{4}$} & \multicolumn{5}{|c|}{$t\left(\hat{\beta}_{4}\right)$} \\
\hline Low & 0.36 & 0.33 & 0.32 & 0.31 & 0.26 & 9.45 & 8.06 & 7.70 & 7.87 & 9.06 \\
\hline$(2)$ & 0.38 & 0.35 & 0.30 & 0.29 & 0.25 & 8.33 & 8.28 & 6.40 & 7.12 & 8.73 \\
\hline (3) & 0.28 & 0.22 & 0.24 & 0.15 & 0.21 & 5.50 & 4.97 & 5.59 & 4.34 & 6.18 \\
\hline (4) & 0.14 & -0.20 & -0.13 & -0.21 & -0.10 & 2.05 & -3.95 & -2.44 & -2.71 & -2.29 \\
\hline High & -0.30 & -0.63 & $\begin{array}{c}-0.74 \\
\hat{\beta}_{5} \\
\end{array}$ & -0.82 & -0.75 & -2.87 & -10.05 & $\begin{array}{c}-11.05 \\
t\left(\hat{\beta}_{5}\right) \\
\end{array}$ & -12.82 & -10.03 \\
\hline Low & 0.18 & 0.09 & 0.18 & 0.22 & 0.21 & 3.33 & 1.82 & 3.14 & 3.64 & 4.62 \\
\hline (2) & 0.08 & 0.07 & 0.06 & 0.13 & 0.18 & 1.33 & 1.34 & 1.03 & 2.30 & 4.79 \\
\hline (3) & 0.13 & 0.00 & -0.00 & 0.15 & 0.16 & 1.93 & 0.07 & -0.08 & 2.63 & 3.42 \\
\hline (4) & -0.02 & -0.11 & 0.03 & 0.13 & 0.01 & -0.24 & -1.66 & 0.40 & 1.68 & 0.21 \\
\hline High & -0.15 & -0.49 & -0.40 & -0.40 & -0.40 & -1.17 & -5.37 & -4.39 & -4.63 & -4.98 \\
\hline
\end{tabular}

The quintile sorts along the column are based on market capitalization while sorts along the rows are based on asset degree. Parameters estimated under the model, $r_{i}-r f=$ $\alpha+\beta_{1} M k t+\beta_{2} S M B+\beta_{3} H M L+\beta_{4} R M W+\beta_{5} C M A+\varepsilon_{i}$. Intercepts $(\alpha)$ are multiplied by 100 , standard errors are reported in parenthesis. Market capitalization (ME) is the number of outstanding shared multiplied by the price, reported in millions of dollars. Estimates are based on 623 consecutive months of data. Standard errors are robust to heteroskedasticity. 
Table 4.5: Long-Short portfolios formed within ME quintiles

\begin{tabular}{lccccc}
\hline \hline $\mathrm{ME}$ & Small & $(2)$ & $(3)$ & $(4)$ & Large \\
\hline$\alpha$ & 1.10 & 0.02 & -0.07 & 0.19 & 0.24 \\
& $(.16)$ & $(.14)$ & $(.15)$ & $(.14)$ & $(.13)$ \\
\hline Mkt & 0.32 & 0.36 & 0.31 & 0.29 & 0.23 \\
& $(.05)$ & $(.04)$ & $(.04)$ & $(.04)$ & $(.03)$ \\
$S M B$ & 0.42 & 0.53 & 0.40 & 0.35 & 0.27 \\
& $(.07)$ & $(.06)$ & $(.06)$ & $(.06)$ & $(.06)$ \\
$H M L$ & -0.55 & -0.63 & -0.64 & -0.52 & -0.39 \\
& $(.10)$ & $(.09)$ & $(.08)$ & $(.08)$ & $(.09)$ \\
$R M W$ & -0.66 & -0.96 & -1.06 & -1.13 & -1.01 \\
& $(.11)$ & $(.08)$ & $(.08)$ & $(.08)$ & $(.09)$ \\
$C M A$ & -0.33 & -0.58 & -0.58 & -0.62 & -0.61 \\
& $(.13)$ & $(.11)$ & $(.11)$ & $(.11)$ & $(.10)$ \\
\hline$R^{2}$ & 0.56 & 0.72 & 0.70 & 0.71 & 0.66 \\
$S R$ & 0.58 & 0.17 & 0.28 & 0.14 & 0.11 \\
\hline
\end{tabular}

Intercepts $(\alpha)$ are multiplied by 100, standard errors are reported in parenthesis. Market capitalization (ME) is the number of outstanding shared multiplied by the price, reported in millions of dollars. Estimates are based on 553 consecutive months of data. Standard errors are robust to heteroskedasticity.

Further we find that the sensitivity to the five Fama-French factors also display variation across connectedness sorts. Within all size quintiles we find that the market sensitivity increase with the level of connectedness. As such the less liquid stocks tend to react stronger to movements in the market. The same monotonic sensitivity increase, from low to high degree sorts, are found with respect to the size factor within all quintiles. Value and Profitability factor sensitivities decrease from the low degree sorts to the high degree sorts. The remaining Investment factor loadings do not follow any consistent pattern across the size portfolio sorts. However, in the fifth quintile these exposures decrease monotonically. In all we want to particularly stress three results. First, our liquidity measure is priced. Second, it measures a phenomenon that is especially prominent among small stocks. Thirdly, these assets are on average more sensitive to market fluctuations.

Table 4.5 presents the evaluation of no-cost portfolio combination. Within each size quintile we form portfolios that hold equal positive weights in the highly connected assets to negative weights among assets with low connectedness. 
Regressing these portfolios onto the Fama-French factors we find that the alpha return premium within the small stock quintile is significant at all conventional significance levels. It amounts to an average monthly premium of 1.10 percent. Additionally we find that this portfolio combination provides an annualized Sharpe ratio of 0.58 . The alpha of the fifth quintile portfolio is not significant at the one percent level.

The above findings confirm our initial result stating that the connectedness premium is not solely conflating effects of low market capitalization. Particularly for portfolios of only small stocks, the gains from the connectedness measure remain economically clear and statistically significant. Additionally, we find that while factor exposures vary a lot within each size quintiles, they are relatively stable across portfolios of similar levels of connectedness. Thus, much of the heterogeneity we observe is due to differences in our constructed measure of asset connectedness and not due to market capitalization.

\subsubsection{Market connectedness}

Asset connectedness is a characteristic that can be used to form portfolios directly. Market connectedness, however, is an aggregate characteristic of the network and does not directly provide portfolio sorts. To evaluate the market connectedness as a factor we form the portfolio sorts on the absolute sensitivity to innovations in the market connectedness. Treating market connectedness as a proxy for the network diameter, we measure the aggregate impact of informational dissemination through order flows. Asset returns that are highly sensitive to these adjustments are thus less liquid than assets that are less sensitive. We define the diameter innovations as the residuals of an $\mathrm{AR}(1)$ filter.

Portfolio sorts are formed using a two-step procedure. First, we estimate residual returns, in excess to the risk-free rate, conditional on a rolling window of the five Fama-French factors. Estimates start from a minimum window length of three years of monthly data. The window grows to at most include the previous five years. Using the residual returns, $\tilde{r}$, we estimate the asset sensitivities to the market connectedness denoted by $\bar{d}_{t}$. The second stage estimates the asset sensitivities $\beta^{*}$ under the same rolling window framework applied in the first stage.

$$
\tilde{r}_{i, t}=\beta_{0, t}+\beta_{i, t}^{*} \bar{d}_{t}+u_{i, t}
$$


Table 4.6: Decile portfolio sorts based on market diameter

\begin{tabular}{lcccccccccc}
\hline \hline & $(1)$ & $(2)$ & $(3)$ & $(4)$ & $(5)$ & $(6)$ & $(7)$ & $(8)$ & $(9)$ & $(10)$ \\
\hline $\mathrm{N}$ & 93 & 88 & 96 & 97 & 105 & 108 & 116 & 126 & 149 & 217 \\
$\mathrm{ME}$ & 673 & 690 & 709 & 701 & 702 & 698 & 706 & 702 & 702 & 715 \\
\hline $\bar{r}$ & 1.01 & 0.96 & 0.91 & 0.88 & 0.99 & 1.04 & 1.04 & 1.00 & 1.06 & 1.23 \\
$\alpha$ & 0.14 & 0.11 & 0.08 & 0.05 & 0.14 & 0.17 & 0.18 & 0.10 & 0.20 & 0.36 \\
& $(.06)$ & $(.07)$ & $(.06)$ & $(.06)$ & $(.06)$ & $(.06)$ & $(.06)$ & $(.06)$ & $(.06)$ & $(.07)$ \\
\hline \multirow{2}{*}{$M k t$} & 1.01 & 0.99 & 0.98 & 0.99 & 1.01 & 1.04 & 1.04 & 1.07 & 1.09 & 1.17 \\
& $(.02)$ & $(.02)$ & $(.02)$ & $(.02)$ & $(.02)$ & $(.02)$ & $(.02)$ & $(.02)$ & $(.02)$ & $(.02)$ \\
$S M B$ & 0.33 & 0.29 & 0.33 & 0.34 & 0.34 & 0.34 & 0.39 & 0.40 & 0.43 & 0.56 \\
& $(.03)$ & $(.03)$ & $(.02)$ & $(.03)$ & $(.03)$ & $(.03)$ & $(.02)$ & $(.03)$ & $(.03)$ & $(.04)$ \\
$H M L$ & 0.32 & 0.32 & 0.29 & 0.35 & 0.33 & 0.28 & 0.31 & 0.25 & 0.24 & 0.17 \\
& $(.05)$ & $(.04)$ & $(.04)$ & $(.05)$ & $(.04)$ & $(.05)$ & $(.04)$ & $(.05)$ & $(.04)$ & $(.05)$ \\
$R M W$ & 0.24 & 0.29 & 0.25 & 0.23 & 0.26 & 0.27 & 0.21 & 0.24 & 0.19 & 0.04 \\
& $(.04)$ & $(.04)$ & $(.04)$ & $(.04)$ & $(.04)$ & $(.04)$ & $(.03)$ & $(.05)$ & $(.05)$ & $(.06)$ \\
$C M A$ & 0.12 & 0.08 & 0.10 & 0.03 & 0.05 & 0.10 & 0.05 & 0.17 & 0.06 & 0.04 \\
& $(.05)$ & $(.06)$ & $(.05)$ & $(.06)$ & $(.06)$ & $(.05)$ & $(.05)$ & $(.06)$ & $(.07)$ & $(.07)$ \\
\hline$R^{2}$ & 0.92 & 0.92 & 0.93 & 0.92 & 0.92 & 0.93 & 0.94 & 0.93 & 0.94 & 0.94 \\
\hline
\end{tabular}

Intercepts $(\alpha)$ are multiplied by 100, standard errors reported in parenthesis. The raw returns are denoted $\bar{r}$ and are multiplied by 100 . Market capitalization (ME) is the number of outstanding shared multiplied by the price, reported in millions of dollars. Estimates are based on 553 consecutive months of data. Standard errors are robust to heteroskedasticity.

Again, an asset $i$ is assigned into a portfolio sort in time $t$ based on the ranking of its absolute $\beta^{*}$ in $t-1$. Just as with the portfolio sorts on connectedness we strive to maintain similar levels of market capitalization across the decile sorts. Due to the rolling window aspect of this two-stage estimation procedure we are unable to form portfolio sorts in the first five years of the time frame.

Table 4.6 reports the number of assets, market capitalization, conditional returns and parameter estimates of the Fama-French five factor model. Similar to the sorts formed on asset connectedness, we find that the assets that exhibit high absolute sensitivity to the market diameter on average have lower market capitalization. In contrast to the connectedness sorts, however, the diameter portfolios are generally larger and with less idiosyncratic noise.

Along the same lines as before we form a no-cost portfolio of a long position in the highly exposed assets and a short position in the assets with low exposures. Table 4.6 displays this portfolios exposures to the five factors along with its conditional return. We find that this portfolio contains more idiosyncratic noise than 
Table 4.7: Long-Short portfolio sorts based on market connectedness

\begin{tabular}{ccccccccr} 
& $\alpha$ & $M k t$ & $S M B$ & $H M L$ & $R M W$ & $C M A$ & $R^{2}$ & $S R$ \\
\hline$(10)-(1)$ & 0.22 & 0.16 & 0.23 & -0.15 & -0.21 & -0.07 & 0.49 & 0.34 \\
& $(.07)$ & $(.02)$ & $(.04)$ & $(.05)$ & $(.05)$ & $(.08)$ & & \\
& &
\end{tabular}

Intercepts $(\alpha)$ are multiplied by 100, t-statistics are reported in parenthesis. Market capitalization (ME) is the number of outstanding shared multiplied by the price, reported in millions of dollars. The Sharpe ratio (SR) is annualized using a $\sqrt{12}$ scaling. Estimates are based on 553 consecutive months of data. Standard errors are robust to heteroskedasticity.

any other portfolio sort so far. Yet, despite this, the monthly excess return to the five factors is statistically significant at the 1 percent level. The point estimate indicate an average monthly excess return of 0.21 percent.

In sum, the estimated networks based on trade-pricing relations provides both asset and market characteristics both of which provide portfolio sorts with positive returns in excess to the five Fama-French factors. We find that under standard errors that are robust to heteroskedasticity, both returns are statistically significant at conventional levels of significance. Hence, both of these two network features, connectedness and network diameter, are priced factors.

\subsection{Conclusion}

In this chapter we estimate a vast network of assets where cross asset relations are quantified by the pricing sensitivities to order flows. Aggregating these relations conveniently summarizes the degree of informational asymmetry and asset connectivity. Going beyond the individual asset we attempt to form a measure of market liquidity through changes in the average connectedness, which proxy for adjustments in the network diameter.

Our main challenge is in the estimation of the asset neighborhoods that form the network. Updating the network monthly only affords the estimator a small number of observations whereas the number of potential neighbors is vast. To overcome this challenge we rely on statistical regularization. Making use of an elastic net we promote neighborhoods including only a subset of the market, yet accounting for highly correlated order flows.

Using data from 1965 until 2017, collected from CRSP, we establish monthly networks. With these networks we compute the distribution of asset connectedness and the market connectedness. The market connectedness varies over time in accordance with periods of financial distress. Times that are seen as periods of highly illiquid markets, such as 1987 'Black Monday' and the financial crisis of 
2008, are associated with very high levels of market connectedness. This implies that markets at these times are highly sensitive to adjustments in order flows. On the basis of individual assets we find that connectedness to our trade networks is a priced source of risk. Highly connected assets are less liquid, the risk that follows is assigned a return premium. This return premium is found in excess to the well known Fama-French factors and is statistically significant. Disentangling the implied size effects from the liquidity premium we find that our connectedness measure still provides robust return premiums. These premium are present among small stocks in particular, which display alphas that are monotonically increasing in the level of illiquidity. In all, our results paint a picture of our measure as capturing a phenomena that is prominent for very small stocks that are also very sensitive to market fluctuations.

Further, we find that while the magnitude and characteristics of order flows have greatly changed since the mid 90s' the economic function in transmitting news and information appears robust. The asset connectedness remains a priced source of risk both before and after this regime shift. It appears, however, that the size of the excess return is larger in the period that followed since 1995. These results are presented in Appendix 4.B

There are a number of open avenues for future research. Prominent among these is to evaluate whether informational asymmetry can explain some anomalies that remain within the Fama-French five factor model. Fama and French (2015) comment that "Microcap extreme growth stocks ... are a huge problem" for the three factor model, which is only partially alleviated by the additional two factors. Furthermore, there are potential extensions into high-frequency data sets. Increasing the data frequency will potentially allow us to disentangle the permanent from the transitory effect of order flows. This would make it possible to more precisely assess connectivity as well as trade informativeness. 



\section{Appendix}

\section{A Network price reversions}

As movements in network measures of degree and diameter appear to coincide with periods of financial distress we turn to the characteristics of aggregate price reversions. We consider two estimates of the price reversions, the original PastorStambaugh gamma estimate and the gamma conditional on the cross-asset price sensitivities to order flows.

Figure 4.A.1 reveals that the price reversions are strikingly similar. Thus, the Pastor-Stambaugh parameters appears robust to the inclusion of the crosssectional pricing impact. In fact, allowing for cross-asset pricing impacts only induce small adjustments in its sensitivity to its own order flow, the correlation between the two series is 0.95 . However, while the average price reversion appears very noisy, we find that large drops in this average also tend to coincide with financial distress. However, the drops are much less pronounced compared to the general level of variability.

Innovations in the price reversion series, constructed as the residuals of an $\operatorname{AR}(1)$ filter, display great variability around the 1987, early 2000 and 2008 crisis. These are innovations associated with other consequences of informational asymmetry, separate from price sensitivities. We should therefore find that these also coincide with periods of low liquidity despite conditioning on pricing sensitivities. Hence, in addition to the co-movement between the degree and diameter and financial distress we find that the separate but related phenomena of price reversions appears robust against the inclusion of our price sensitivity measures. 
Figure 4.A.1: Price reversions

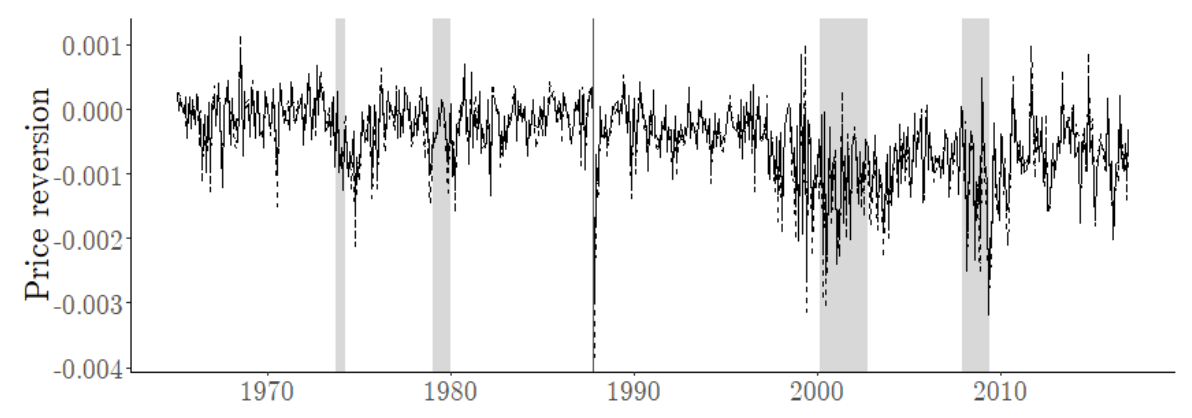

Conditional and unconditional price reversions measured by the average PastorStambaugh gamma. The conditional price reversions (solid line) are estimated while including the cross-asset price sensitivities, as specified in model 4.2. Shaded areas correspond to the first and second oil crisis, 'Black Monday' of 1987, the Dot-com bubble and the Great recession.

\section{B Connectedness before and after 1995}

Following order flows since 1965, presented in Figure 4.1, we find that the 1990s' marks a dramatic shift in the magnitude of order flows on the NYSE. During the years between 1990 and 1995 the average daily order flows largely range between minus two and two million dollars. The same daily averages following the year 2000 often vary in the range of twenty up to forty million dollars. These changes are intuitive since the financial markets have grown in size and technology has enabled faster trade at higher frequency. Whether the new circumstances strengthened or undermined the economic function of order flows is not obvious.

Here we assess the role of connectedness in the trade network before and after 1995. The choice of 1995 as the cutoff between the two regimes is arbitrary. We follow the same portfolio formation strategy as in the previous section. Assets are sorted into decile portfolios, with largely equal market capitalization, based on their network degree. Forming these portfolios and estimating their exposures to the five Fama-French factors we assess the monthly return premium before and after 1995 .

We find, reported in Table 4.B.1, that the return premium appears large in the post-1995 period. Before 1995 we still find that the mean expected return conditional on the Fama-French factors is higher in portfolios of assets with high levels of connectedness. The excess return across these portfolios increase from 
Figure 4.A.2: Price reversion innovation

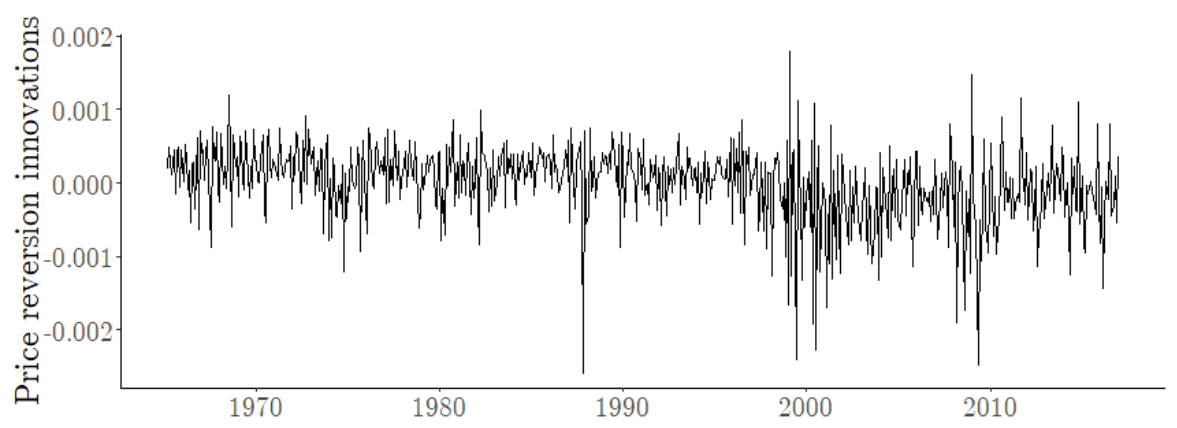

Innovations in price reversions measured by the average Pastor-Stambaugh gamma. The innovations are the residuals of an AR(1) model. Shaded areas correspond to the first and second oil crisis, 'Black Monday' of 1987, the Dot-com bubble and the Great recession.

-0.07 , in the portfolio formed on the least connected assets, to 0.32 percent in the portfolio of the most connected. The same pattern emerges in the period after 1995. The distinction between the periods is in the magnitudes the of expected excess return. The first five decile portfolio sorts produce values of $\alpha$ in the range 0.08 to 0.13 , none significantly different from zero. The highest decile sort, however, gives an significant expected monthly return, in excess of Fama-French factors, of 0.89 percent.

We form no-cost portfolios containing a long position in the highly connected asset and equally sized short position in the assets with low connectedness. Similar to the full sample we find that both the pre and post 1995 periods deliver statistically significant positive returns in excess the what may be accounted for by the other factors. Results are reported in Table 4.B.2.

The average expected return in excess to other factors in the no-cost portfolio following 1995 is 0.77 percent. This is in contrast to before 1995 where the same expected average monthly return was 0.39 percent. Apart from $\alpha$, the exposures to the Fama-French factors appear to have changed with respect to some factors. Both long-short portfolios display similar low sensitivities to the market factor. They are also similar with respect to the value factor and size factors, only displaying minor adjustments. All exposures to these three factors are statistically significant. The profitability and investment factors, however, differs much between the two periods. Both factors load negatively onto our portfolios, yet much more so in the post-95 period. 
Table 4.B.1: Decile portfolio sorts based on asset connectedness

Before 1995

\begin{tabular}{|c|c|c|c|c|c|c|c|c|c|c|}
\hline & (1) & $(2)$ & (3) & $(4)$ & $(5)$ & (6) & (7) & (8) & (9) & (10) \\
\hline $\mathrm{N}$ & 35 & 46 & 59 & 71 & 85 & 99 & 117 & 147 & 193 & 310 \\
\hline ME & 137 & 151 & 151 & 149 & 149 & 150 & 149 & 149 & 149 & 150 \\
\hline$\alpha$ & $\begin{array}{r}-0.07 \\
(.12)\end{array}$ & $\begin{array}{l}0.02 \\
(.08)\end{array}$ & $\begin{array}{l}0.15 \\
(.07)\end{array}$ & $\begin{array}{l}0.19 \\
(.07)\end{array}$ & $\begin{array}{l}0.15 \\
(.07)\end{array}$ & $\begin{array}{l}0.28 \\
(.07)\end{array}$ & $\begin{array}{l}0.27 \\
(.06)\end{array}$ & $\begin{array}{l}0.30 \\
(.06)\end{array}$ & $\begin{array}{l}0.45 \\
(.06)\end{array}$ & $\begin{array}{l}0.32 \\
(.09)\end{array}$ \\
\hline$R^{2}$ & 0.77 & 0.86 & 0.91 & 0.93 & 0.93 & 0.95 & 0.96 & 0.97 & 0.97 & 0.96 \\
\hline \multicolumn{11}{|c|}{ After 1995} \\
\hline $\mathrm{N}$ & 88 & 93 & 100 & 111 & 121 & 141 & 163 & 201 & 266 & 398 \\
\hline ME & 1250 & 1343 & 1323 & 1330 & 1326 & 1317 & 1328 & 1336 & 1330 & 1340 \\
\hline$\alpha$ & $\begin{array}{l}0.13 \\
(.11)\end{array}$ & $\begin{array}{l}0.08 \\
(.08)\end{array}$ & $\begin{array}{l}0.11 \\
(.09)\end{array}$ & $\begin{array}{l}0.13 \\
(.10)\end{array}$ & $\begin{array}{l}0.13 \\
(.08)\end{array}$ & $\begin{array}{l}0.27 \\
(.08)\end{array}$ & $\begin{array}{l}0.28 \\
(.08)\end{array}$ & $\begin{array}{l}0.36 \\
(.07)\end{array}$ & $\begin{array}{l}0.58 \\
(.10)\end{array}$ & $\begin{array}{l}0.89 \\
(.13)\end{array}$ \\
\hline$R^{2}$ & 0.84 & 0.88 & 0.90 & 0.90 & 0.91 & 0.91 & 0.93 & 0.95 & 0.94 & 0.94 \\
\hline
\end{tabular}

Intercepts $(\alpha)$ are multiplied by 100, t-statistics are reported in parenthesis. The estimates of $\alpha$ are conditional on the Fama-French five factor model. Market capitalization (ME) is the number of outstanding shared multiplied by the price, reported in millions of dollars. Estimates in the first panel are based on 359 consecutive months of data. Estimates in the second panel are based on 264 months. Standard errors are robust to heteroskedasticity.

In all it seems like the role of connectedness as measured by the asset network degree is maintained both before and after 1995. The difference between the periods appears to be that the recent period has seen an increased return premium associated with asset connectivity. Hence, even though the magnitude of order flows has greatly increased, the feature measured by our network remains relevant in both periods.

\section{C Erdös and Renyi diameter}

In this section we present the diameter approximation proposed by Erdös and Rényi (1959). This approximation is closely related to our cross-sectional average. Figure 4.C.1 displays the network diameter progressing over time. The diameter of the network vary much over time but appears to align well with recent periods of financial distress. The two major oil crises of the 1970's do not seem to strongly impact the market diameter beyond what is typical variation over this decade. However, whereas the following decade is generally more stable, in line with this period as the great moderation period, October 1987 coincides with a huge drop in 
Table 4.B.2: Long-Short portfolio sorts based on connectedness

\begin{tabular}{|c|c|c|c|c|c|c|c|c|}
\hline \multicolumn{9}{|c|}{ Before 1995} \\
\hline & $\alpha$ & $M k t$ & $S M B$ & $H M L$ & $R M W$ & $C M A$ & $R^{2}$ & $S R$ \\
\hline$(10)-(1)$ & $\begin{array}{l}0.39 \\
(.16)\end{array}$ & $\begin{array}{l}0.36 \\
(.04)\end{array}$ & $\begin{array}{l}0.87 \\
(.06)\end{array}$ & $\begin{array}{r}-0.37 \\
(.09)\end{array}$ & $\begin{array}{r}-0.34 \\
(.14)\end{array}$ & $\begin{array}{r}-0.23 \\
(.15)\end{array}$ & 0.69 & 0.36 \\
\hline \multicolumn{9}{|c|}{ After 1995} \\
\hline$(10)-(1)$ & $\begin{array}{l}0.77 \\
(.19)\end{array}$ & $\begin{array}{l}0.38 \\
(.06)\end{array}$ & $\begin{array}{l}0.68 \\
(.07)\end{array}$ & $\begin{array}{r}-0.44 \\
(.09)\end{array}$ & $\begin{array}{r}-0.97 \\
(.08)\end{array}$ & $\begin{array}{r}-0.71 \\
(.10)\end{array}$ & 0.86 & 0.25 \\
\hline
\end{tabular}

Intercepts $(\alpha)$ are multiplied by 100 , t-statistics are reported in parenthesis. Market capitalization (ME) is the number of outstanding shared multiplied by the price, reported in millions of dollars. The Sharpe ratio (SR) is annualized using a $\sqrt{12}$ scaling. Estimates in the first panel are based on 359 consecutive months of data. Estimates in the second panel are based on 264 months. Standard errors are robust to heteroskedasticity.

market diameter. Similarly we find that the last years of the twentieth century and the years following 2008 are both marked by low network diameter. In connection to the economic reasoning presented previously, these findings imply that these are periods in which informational asymmetry is comparably large.

Figure 4.C.1: Network diameter

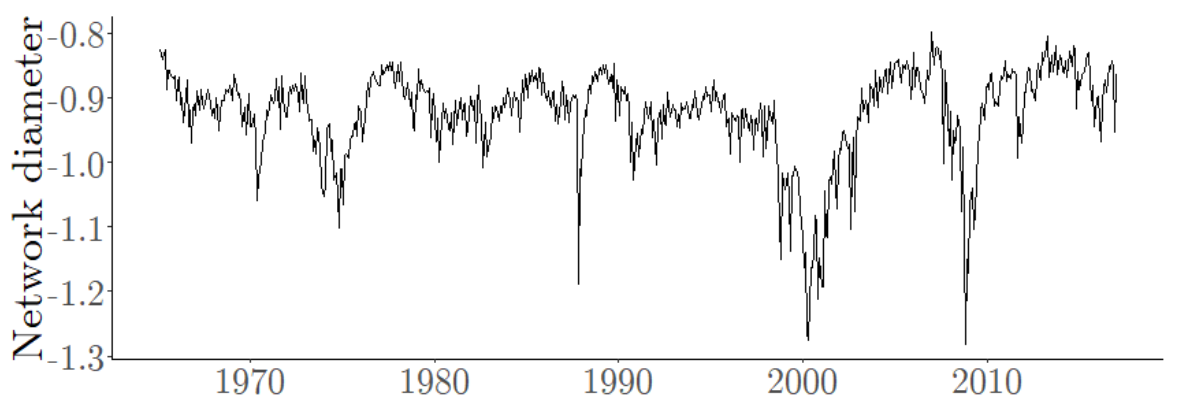

Monthly estimates of trade network diameter over time. Shaded areas correspond to the first and second oil crisis, 'Black Monday' of 1987, the Dot-com bubble and the Great recession.

Innovations, news, in the market diameter are computed under an $\mathrm{AR}(1)$ filter. The innovations, Figure 4.C.2, reveal that the three periods of greatest turbulence are (1) 'Black Monday' of 1987, (2) the years preceding and following 2000, and (3) the years associated with the 'Great recession'. These are periods of great financial distress and financial liquidity was considered low, thus it appears that 
Figure 4.C.2: Erdös Renyi diameter innovations

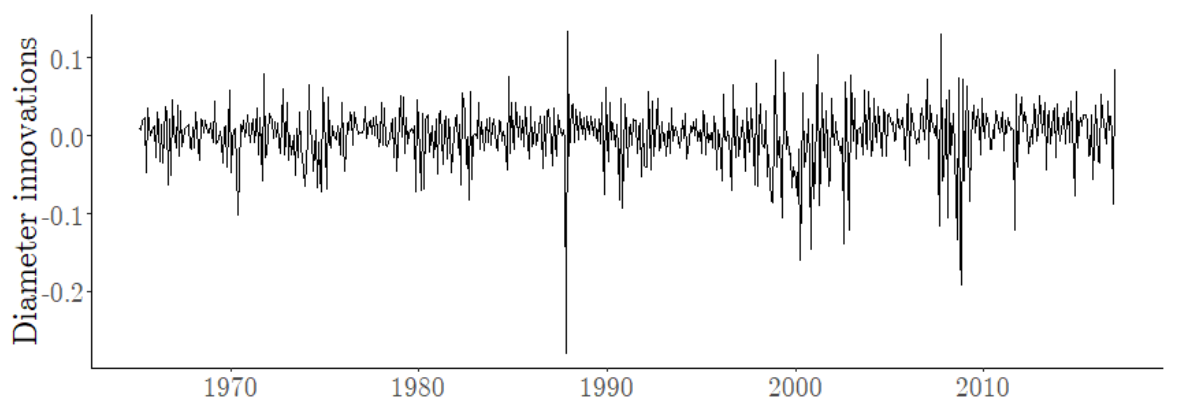

Innovations in estimated Erdös Renyi diameter. The innovations are the residuals of an $\operatorname{AR}(1)$ model.

market diameter does capture relevant information connected to aggregate liquidity. Beyond these periods the variance of the news is comparably stable. Since the variation as expected follows along the same lines of the cross-sectional average it follows that we should not find any discernible difference between this measure and the average for asset pricing purposes. Table (4.C.1) presents the results of forming the decile sorts based on absolute price sensitivity to the Erdös-Renyi diameter, along the same lines as the results presented in section 4.4.

Table 4.C.1: Long-Short portfolio sorts based on Erdös-Renyi diameter

\begin{tabular}{rccccccrr} 
& $\alpha$ & $M k t$ & $S M B$ & $H M L$ & $R M W$ & $C M A$ & $R^{2}$ & $S R$ \\
\hline$(10)-(1)$ & 0.22 & 0.15 & 0.25 & -0.12 & -0.27 & -0.06 & 0.54 & 0.33 \\
& $(.07)$ & $(.02)$ & $(.03)$ & $(.04)$ & $(.04)$ & $(.06)$ & &
\end{tabular}

Intercepts $(\alpha)$ are multiplied by 100, t-statistics are reported in parenthesis. Market capitalization (ME) is the number of outstanding shared multiplied by the price, reported in millions of dollars. The Sharpe ratio (SR) is annualized using a $\sqrt{12}$ scaling. Estimates are based on 553 consecutive months of data. Standard errors are robust to heteroskedasticity.

Table 4.C.2 reports the number of assets, market capitalization, conditional returns and parameter estimates of the Fama-French five factor model. Similar to the sorts formed on asset connectedness, we find that the assets that exhibit high absolute sensitivity to the market diameter on average have lower market capitalization. In contrast to the connectedness sorts, however, the diameter portfolios are generally larger and with less idiosyncratic noise. 
Table 4.C.2: Decile portfolio sorts based on market Erdös-Renyi diameter

\begin{tabular}{lcccccccccc}
\hline \hline & $(1)$ & $(2)$ & $(3)$ & $(4)$ & $(5)$ & $(6)$ & $(7)$ & $(8)$ & $(9)$ & $(10)$ \\
\hline $\mathrm{N}$ & 95 & 95 & 100 & 103 & 101 & 103 & 109 & 122 & 151 & 213 \\
$\mathrm{ME}$ & 668 & 703 & 706 & 697 & 701 & 698 & 701 & 705 & 704 & 715 \\
\hline$\alpha$ & 0.14 & 0.07 & 0.16 & 0.11 & 0.17 & 0.10 & 0.12 & 0.22 & 0.20 & 0.36 \\
& $(.06)$ & $(.06)$ & $(.06)$ & $(.06)$ & $(.06)$ & $(.06)$ & $(.06)$ & $(.06)$ & $(.06)$ & $(.07)$ \\
\hline \multirow{2}{*}{$M k t$} & 1.02 & 1.01 & 1.00 & 1.00 & 1.02 & 1.02 & 1.04 & 1.04 & 1.08 & 1.16 \\
& $(.02)$ & $(.02)$ & $(.02)$ & $(.02)$ & $(.02)$ & $(.02)$ & $(.02)$ & $(.02)$ & $(.02)$ & $(.02)$ \\
$S M B$ & 0.34 & 0.34 & 0.31 & 0.34 & 0.33 & 0.31 & 0.33 & 0.41 & 0.46 & 0.58 \\
& $(.03)$ & $(.03)$ & $(.03)$ & $(.03)$ & $(.03)$ & $(.03)$ & $(.03)$ & $(.03)$ & $(.03)$ & $(.04)$ \\
$H M L$ & 0.30 & 0.30 & 0.30 & 0.30 & 0.33 & 0.25 & 0.31 & 0.29 & 0.29 & 0.18 \\
& $(.04)$ & $(.03)$ & $(.04)$ & $(.04)$ & $(.04)$ & $(.05)$ & $(.04)$ & $(.04)$ & $(.04)$ & $(.06)$ \\
$R M W$ & 0.28 & 0.28 & 0.26 & 0.26 & 0.25 & 0.26 & 0.22 & 0.17 & 0.18 & 0.01 \\
& $(.03)$ & $(.04)$ & $(.04)$ & $(.04)$ & $(.05)$ & $(.04)$ & $(.05)$ & $(.04)$ & $(.04)$ & $(.05)$ \\
$C M A$ & 0.12 & 0.08 & 0.09 & 0.09 & 0.06 & 0.14 & 0.03 & 0.06 & 0.02 & 0.06 \\
& $(.05)$ & $(.05)$ & $(.06)$ & $(.05)$ & $(.06)$ & $(.05)$ & $(.06)$ & $(.06)$ & $(.06)$ & $(.07)$ \\
\hline$R^{2}$ & 0.93 & 0.93 & 0.92 & 0.92 & 0.93 & 0.93 & 0.93 & 0.93 & 0.94 & 0.94
\end{tabular}

Intercepts $(\alpha)$ are multiplied by 100, t-statistics are reported in parenthesis. Market capitalization $(\mathrm{ME})$ is the number of outstanding shared multiplied by the price, reported in millions of dollars. Estimates are based on 553 consecutive months of data. Standard errors are robust to heteroskedasticity. 
Comparing these results to the outcomes under the decile sorts based on the sensitivity to the cross-sectional average, reported in Section 4.4, there is hardly any discernible difference. 
Chapter 5

\section{Conclusion}

"Out, out, brief candle!"

- Macbeth, 5.5.24, (William Shakespeare 1564 - 1616) 
Statistical methods designed to handle excessive model complexity now provides a big toolbox for economists. While we still do not understand the full set of statistical properties of all of these estimators, we do now understand much about how methods like the lasso, ridge, elastic net, boosting perform asymptotically. This provides great opportunities for economics. My thesis have broadly explored our high-dimensional toolbox in service of two purposes. In chapters 2 and 3 we have made use of these methods to translate fundamental financial exercises into large data environments. In chapter 4 we make use of the toolbox to extract information from a large data environment, to improve the understanding of a fundamental financial exercise.

My second chapter provide some important insights for empirical asset pricing. We propose a novel usage of the Hansen-Jagannathan distance, which is to decompose the estimation into a time-series instrumental variable design. This enables us to study asset pricing models in an environment where the number of returns exceed the amount of historical data. Economically this offers opportunities to estimate and compare economic factor models while also maintaining that assets can display a large variety of characteristics. Our two-stage framing is also beneficial for our understanding of the econometric properties of our method, we are able to provide consistent risk price estimates and standard errors. The empirical application of our method confirms the common conception that macro economic factor models display low predictive power for the cross-section of returns. However, we also find that there are very large relative gains to be made from including piece-wise linear terms in classic macroeconomic factors. It is conceivable that macroeconomic factors actually hold considerable predictive power but that asset exposures to macroeconomic circumstances might be highly non-linear. This is beyond the scope of my dissertation but an excellent avenue for future research.

In the third chapter of my thesis I propose an approach to minimum-variance optimization that draws upon tools from high-dimensional statistics combined with prominent financial factors. I estimate the inverse covariance matrix directly by using the Fama-French factors to predict common variation among returns and the graphical lasso to form sparse hedging portfolios that account for partial correlation between residuals to the factor structure. The empirical performance is striking, the method lowers portfolio volatility more than the competing methods. A bootstrapping exercise confirms that this volatility reduction is statistically significant. I find that much of the gains follow from the usage of the graphical lasso, which removes the need to perform a large-scale matrix inversion. This approach 
offers much in terms of future research. Among other things, future research should work to incorporate our understanding of the reliability of volatilites and covariances in the regularization.

The fourth chapter of my thesis targets the financial exposure to illiquidity from asymmetric information. We make use of the elastic net to extract estimates of the pricing impacts from the large cross-section of order flows on the market. We view the cross-asset relations in terms of a financial network of price-trade relations. From this intuition we form measures of illiquidity that correspond to the degree of connectedness an asset displays to the network. We find that this connectedness is priced, and so is the exposure to adjustments in the average connectedness. In particular we find that the characteristics we construct are predictive of the expected returns among assets with low market capitalization. This finding conforms with the common perception that illiquidity is a small stock phenomena. The pressing questions that remain is what can we gain from incorporating higher frequencies of informational flows and how much more can we learn by parameterizing the potential dynamics of these variables.

Concluding, regularized estimators makes it possible for us to extract information, which is predictive of expected returns, from a vast numbers of order flows. I have also found that the Hansen-Jagannathan distance provides an estimation framework in which workhorse methods from machine learning can provide effective and flexible risk price estimation. Furthermore, I found that graphical model selection can perform portfolio allocation that is especially well suited to approach segmented markets. Lastly, in the broadest sense the conclusion to my Ph.D. is that the large cross-section of returns both contain some missing pieces to our economic puzzles, but also some new puzzles. What stands out, however, is that the combination of fundamental economic structures together with statistical regularization is mutually beneficial. Data-driven learning can be applied to compensate for the weaknesses in the economic theory, while economic theory can provide a structure to guide the learning. 



\section{Valorization}

"The improvement of understanding is for two ends: first, our own increase of knowledge; secondly, to enable us to deliver that knowledge to others."

- John Locke (1632-1704) 
The research presented herein is about empirical methodology for asset pricing and portfolio management. Within this broad economic context I have pursued a particular focus on issues of portfolio variance reduction, macroeconomic risk factor assessment and financial liquidity risk. These topics are fundamental within the context of academic financial research, but they are also relevant for professionals in the financial sector as well as regulators and supervisory agencies. In this addendum I will attempt to frame and provide examples of the relevance from both of these perspectives. I present this through both the methodological implications of my work and what my empirical findings indicate.

The economic content that unifies the chapters of this thesis is different notions of financial risk. In particular I have studied three facets of risk; (1) macroeconomic risk, (2) liquidity risk and (3) co-movement and financial connectivity. The econometric content that ties together the applications is the challenge of estimating relations between many co-varying variables within very large systems. Thus the methodology proposed is tailored to handle circumstances where either (1) the risk relations are very complicated or (2) the number of financial variables is very large. To achieve this I explore in what manner methods from machine learning and high-dimensional statistics can augment traditional econometric techniques. As such, in the most general sense, these approaches offer opportunities to explore economic issues utilizing more data than was previously possible.

\section{Macroeconomic risk and Portfolio volatility}

At the core of portfolio optimization is the trade-off between risk and expected returns. With respect to the financial sector the economic relevance of this thesis can be recognized through two contributions. First is through the methodology developed for portfolio volatility reduction. Standard methods of minimum variance allocation quickly deteriorates when the number of assets in a portfolio grows large. The method I propose in chapter 3 is well suited to handle portfolios with a potentially very large number of assets. This means that even very large financial portfolios can be made more stable than was previously possible with standard econometric tools. The second contribution I would highlight is the approach proposed to assess and compare models of economic risk. There is a wealth of theory to describe sources of macroeconomic risk. The method presented in chapter 2 is well suited to assess the relative fit of the respective theoretical models even for a large number of assets where traditional econometrics methods would be ill-suited. 
Apart from the relevance of these methodological contribution several empirically important results stand out. For example, that liquidity risk associated with price sensitivity is very prevalent among assets with low market capitalization. From an investment perspective this finding takes the form of a return premium, which can be the basis for a multitude of trading strategies.

With regards to the econometrics presented herein, both finance professionals and economic consultancies have been exploring methods of machine learning and deep learning to optimize consumer products, marketing and pricing. In these sectors the utilization of large amounts of data is a topic that has been a leading frontier for prediction and forecasting. By nesting these methods in an economic and econometric framework this thesis can help move these applications one step closer to our economic questions beyond prediction. The virtue of this is that statistical regularization and high-dimensional statistics do not have to be substitutes to economic models. Rather, it appears that these methods can be mutually beneficial.

\section{Financial stability and co-movement}

Beyond the financial sector, both regulatory authorities and supervisory agencies continuously monitor the financial systems risk exposure to maintain stability. While writing this section, the international financial regulatory framework Basel III is under implementation. Among the pillars of the new framework is the regulation of the ratio of liquid assets to the total flow of liquidity within a financial institution. A critical element for the prospects of this regulation is the precision of the empirical measures of financial liquidity. Chapter 4 of this thesis approaches this issue of financial liquidity and expands upon some of the prominent measures.

Regulators that monitor the stability of the financial system also require a thorough picture of what economic factors are critical for the financial markets and its major institutions. The same methodology proposed herein for portfolio allocation can be applied to understand financial connectivity and spillovers. Whereas is it practical and common to rely on ad hoc identification of the paramount institutions or financial groupings, the methods presented in chapter 3 and 4 introduces a data-driven manner to complement the economic models regulators rely on. An example of how this might take shape is through the perspective of financial liquidity. Many of the prominent measures of financial liquidity omit effects of contemporaneous incidents on financial markets. This means that liquidity such 
that it is regulated may not be properly reflective of ease of trade when markets are in crisis. Using tools we have explored it is possible to augment these measures to make them more adaptable to times of financial distress.

Furthermore, chapter 2 introduces a methodology to compare what sources of macroeconomic risk are most predictive of price adjustments. Thus, this offers an opportunity to predict which factors financial markets are most sensitive to. In turn, this can be used to classify and compare different sources of risk when monitoring the financial system.

\section{In sum}

The research presented in this thesis is relevant for both professionals in the financial sector and regulators. The relevance is related to the monitoring and management of different facets of financial risk. The prospects involve lower portfolio volatility and more precise measures of financial risk. It achieves this through the application of methods that draw upon advancements in other academic disciplines. Approaching traditional economic topics with a novel empirical approach can reveal new aspects to traditional economic phenomena. Whether the methods I have explored will contribute to such a new perspective remains to be determined.

This framing of research in terms of its immediate applicability and relevance outside of academia yet this might not render a complete reflection of the impact of the work. Academia is an important institution to critically assess what we believe to be true and to educate. We do this both through our research and through teaching. My education as a Ph.D. has also contributed to the education of many undergraduate students. Economics is to a great extent an empirical social science and the role of tutors and lecturers teaching empirical methodology is particularly important. The relevance of my research therefore partially extends to the degree with which my understanding helped form more critical and empirically well-versed economists. 


\section{Bibliography}

Acharya, V. V. and L. H. Pedersen (2005): "Asset pricing with liquidity risk," Journal of financial Economics, 77, 375-410.

Ang, A., R. Hodrick, Y. Xing, And X. Zhang (2006): "The Cross-Section of Volatility and Expected Returns," Journal of Finance, 61, 259-299.

Aretz, K., S. M. Bartram, And P. F. Pope (2010): "Macroeconomic risks and characteristic-based factor models," Journal of Banking 83 Finance, 34, 1383-1399.

BAI, J. AND S. NG (2002a): "Determining the Number of Factors in Approximate Factor Models," Econometrica, 70, 191-221.

- (2002b): "Determining the Number of Factors in Approximate Factor Models," Econometrica, 70, 191-221.

- (2013): "Principal Components Estimation and Identification of Static Factors," Journal of Econometrics, 176, 18-29.

Baker, M., B. Bradley, and J. Wurgler (2011): "Benchmarks as limits to arbitrage: Understanding the low-volatility anomaly," Financial Analysts Journal, 67, 40-54.

BAlduzzi, P. And C. RoвotTi (2008): "Mimicking portfolios, economic risk premia, and tests of multi-beta models," Journal of Business 83 Economic Statistics, $26,354-368$.

BAsturk, N. And S. O. R. LÖNn (2018): "Order flow, commonalities and liquidity in vast trade networks," Maastricht University Working Papers.

Belloni, A., D. Chen, V. Chernozhukov, and C. Hansen (2012): "Sparse models and methods for optimal instruments with an application to eminent domain," Econometrica, 80, 2369-2429. 
Berk, J. B. (1995): "A critique of size-related anomalies," The Review of Financial Studies, 8, 275-286.

Borgatti, S. P. And M. G. Everett (2006): "A graph-theoretic perspective on centrality," Social networks, 28, 466-484.

Breiman, L. (1998): "Arcing Classifier (with discussion)," Annals of Statistics, 26, 801-849.

Bryzgalova, S. (2016): "Spurious factors in linear asset pricing models," LSE manuscript.

Bühlmann, P. (2006): "Boosting for high-dimensional linear models," The Annals of Statistics, 34, 559-583.

Bühlmann, P. And S. Van De Geer (2011): Statistics for High-Dimensional Data, Springer.

Caballe, J. and M. Krishnan (1994): "Imperfect competition in a multisecurity market with risk neutrality," Econometrica (1986-1998), 62, 695.

CAI, T., W. LIU, AND X. Luo (2011): "A constrained âĎŞ 1 minimization approach to sparse precision matrix estimation," Journal of the American Statistical Association, 106, 594-607.

Campbell, J. Y., S. J. Grossman, and J. Wang (1993): "Trading volume and serial correlation in stock returns," The Quarterly Journal of Economics, 108, 905-939.

Caner, M., E. Ulasan, L. Callot, and A. Ö. Önder (2017): "A Relaxed Approach to Estimating Large Portfolios and Gross Exposure," Working Paper.

Cespa, G. and T. Foucault (2014): "Illiquidity contagion and liquidity crashes," The Review of Financial Studies, 27, 1615-1660.

Chamberlain, G. and M. Rothschild (1983): "Arbitrage, Factor Structure, and Mean-Variance Analysis on Large Asset Markets," Econometrica, 51, 1281304.

Chen, N., R. Roll, and S. Ross (1986): "Economic Forces and the Stock Market," Journal of Business, 59, 383-403.

Chinco, A. M., A. D. Clark-Joseph, and M. Ye (2017): "Sparse signals in the cross-section of returns," Tech. rep., National Bureau of Economic Research.

Chowdhry, B. And V. NAnda (1991): "Multimarket trading and market liquidity," The Review of Financial Studies, 4, 483-511.

Clarke, R., H. de Silva, and S. Thorley (2011): "Minimum Variance Porfolio Composition," Journal of Portfolio Management, 37, 31-45.

Cochrane, J. H. (2005): Asset Pricing, Princeton University Press. 
(2011): "Presidential address: Discount rates," The Journal of finance, 66, $1047-1108$.

(2017): "Macro-finance," Review of Finance, 21, 945-985.

DeMiguel, V., L. Garlappi, and R. Uppal (2007): "Optimal versus Naive Diversification: How Inefficient Is the 1/N Portfolio Strategy?" Review of Financial Studies, 22, 1915-1953.

DeMiguel, V., A. Martin-Utrera, F. J. Nogales, and R. Uppal (2018): "A portfolio perspective on the multitude of firm characteristics," Working Paper.

Diebold, F. X. And K. Yilmaz (2014): "On the network topology of variance decompositions: Measuring the connectedness of financial firms," Journal of Econometrics, 182, 119-134.

ERdös, P. And A. RÉnyi (1959): "On random graphs I," Publ. Math. Debrecen, 6, 290-297.

Fama, E. And J. MacBeth (1973): "Risk, Return and Equilibrium: Empirical Tests," Journal of Political Economy, 71, 607-636.

FAma, E. F. AND K. R. FRENCH (1993): "Common risk factors in the return of stocks and bonds," Journal of Financial Economics, 33, 3-56.

- (2015): “A Five-Factor Asset-Pricing Model," Journal of Financial Economics, 116, 1-22.

FAN, J., Y. FAN, AND J. LV (2008): "High dimensional covariance matrix estimation using a factor model," Journal of Econometrics, 147, 186-197.

FAn, J., Y. LiaO, AND M. Mincheva (2013): "Large covariance estimation by thresholding principal orthogonal complements," Journal of the Royal Statistical Society: Series B (Statistical Methodology), 75, 603-680.

FAn, J., Y. LiaO, AND X. SHi (2015): "Risks of large portfolios," Journal of econometrics, 186, 367-387.

FAN, J., H. LIU, AND W. WANG (2018): "Large covariance estimation through elliptical factor models," Annals of statistics, 46, 1383.

Feng, G., S. Giglio, And D. XiU (2017): "Taming the Factor Zoo," Working Paper, Booth School of Business, University of Chicago.

- (2019): "Taming the Factor Zoo: A Test of New Factors," Fama-Miller Working Paper, 17-04.

Fleming, J., C. Kirby, And B. Ostdiek (2001): "The economic value of volatility timing," The Journal of Finance, 56, 329-352. 
(2003): "The economic value of volatility timing using âĂIJrealizedâĂ volatility," Journal of Financial Economics, 67, 473-509.

Foygel, R. And M. Drton (2010): "Extended Bayesian Information Criteria for Gaussian Graphical Models," in Advances in Neural Information Processing Systems 23, ed. by J. D. Lafferty, C. K. I. Williams, J. Shawe-Taylor, R. S. Zemel, and A. Culotta, Curran Associates, Inc., 604-612.

Freyberger, J., A. Neuhierl, And M. Weber (2017): "Dissecting characteristics nonparametrically," Tech. rep., National Bureau of Economic Research.

Friedman, J. (2001): "Greedy Function Approximation: A Gradient Boosting Algorithm," Annals of Statistics, 29, 1189-1232.

Friedman, J., T. Hastie, and R. Tibshirani (2008): "Sparse inverse covariance estimation with the graphical lasso," Biostatistics, 9, 432-441.

Garlappi, L., R. Uppal, and T. Wang (2007): "Portfolio Selection with Parameter and Model Uncertainty: A Multi-Prior Approach," Review of Financial Studies, 20, 41-81.

Goto, S. AND Y. XU (2015): "Improving mean variance optimization through sparse hedging restrictions," Journal of Financial and Quantitative Analysis, 50, 1415-1441.

Gu, S., B. T. Kelly, And D. Xiu (2018): "Empirical Asset Pricing Via Machine Learning," .

Hallin, M., C. Mathias, H. Pirotte, and D. Veredas (2011): "Market liquidity as dynamic factors," Journal of Econometrics, 163, 42-50.

Hansen, L. and R. Jagannathan (1997): "Assessing Specification Errors in Stochastic Discount Factor Models," Journal of Finance, 52, 557-590.

Harvey, C. R., Y. Liu, AND H. Zhu (2016): "âĂę and the cross-section of expected returns," The Review of Financial Studies, 29, 5-68.

Hasbrouck, J. (1991a): "Measuring the information content of stock trades," The Journal of Finance, 46, 179-207.

_ (1991b): "The Summary Informativeness of Stock Trades: An Econometric Analysis," Review of Financial Studies, 4, 571-595.

Hasbrouck, J. AND D. J. SEPPi (2001): "Common factors in prices, order flows, and liquidity," Journal of financial Economics, 59, 383-411.

Hastie, T., R. Tibshirani, and J. Friedman (2009): The Elements of Statistical Learning: Data Mining, Inference, and Prediction, Springer series in statistics, Springer. 
Hautsch, N., L. M. Kyj, And R. C. Oomen (2012): "A blocking and regularization approach to high-dimensional realized covariance estimation," Journal of Applied Econometrics, 27, 625-645.

Huberman, G. and S. Kandel (1987): "Mean-Variance Spanning," Journal of Finance, 42, 873-888.

Jagannathan, R. And T. Ma (2003): "Risk reduction in large portfolios: Why imposing the wrong constraints helps," The Journal of Finance, 58, 1651-1683.

Jobson, D. And R. Korkie (1982): "Potential Performance and Tests of Portfolio Efficiency," Journal of Financial Economics, 10, 433-466.

Jobson, J. And B. Korkie (1983): "Sta tistical Inference in Two-Parameter Portfolio Theory with Multiple Regression Software," Journal of Financial and Quantitative Analysis, 18, 189-197.

Kan, R. and C. Zhang (1999): "Two-Pass Tests of Asset Pricing Models with Useless Factors," Journal of Finance, 54, 203-235.

KAN, R. AND G. ZHOU (2007): "Optimal portfolio choice with parameter uncertainty," Journal of Financial and Quantitative Analysis, 42, 621-656.

Kelly, B., S. Pruitt, And Y. Su (2018a): "Characteristics are covariances: A unified model of risk and return," Tech. rep., National Bureau of Economic Research.

(2018b): "Characteristics are covariances: A unified model of risk and return," Tech. rep., National Bureau of Economic Research.

Kleibergen, F. (2009): "Tests of Risk Premia in Linear Factor Models," Journal of Econometrics, 149, 149-173.

Kleibergen, F. And Z. Zhan (2018): "Identification-robust inference on risk premia of mimicking portfolios of non-traded factors," Journal of Financial Econometrics, 16, 155-190.

Korajczyk, R. A. And R. SAdka (2008): "Pricing the commonality across alternative measures of liquidity," Journal of Financial Economics, 87, 45-72.

KozaK, S., S. Nagel, and S. Santosh (2017): "Shrinking the cross section," Tech. rep., National Bureau of Economic Research.

Kyle, A. S. (1985): "Continuous auctions and insider trading," Econometrica: Journal of the Econometric Society, 1315-1335.

Lamont, O. (2001): "Economic Tracking Portfolios," Journal of Econometrics, 105, 161-184. 
Ledoit, O. And M. Wolf (2003): "Improved Estimation of the Covariane Matrix of Stock Returns with an Application to Portfolio Selection," Journal of Empirical Finance, 10, 603-621.

- (2004): "A well-conditioned estimator for large-dimensional covariance matrices," Journal of multivariate analysis, 88, 365-411.

_ (2012): "Nonlinear shrinkage estimation of large-dimensional covariance matrices," The Annals of Statistics, 40, 1024-1060.

Lewellen, J., S. Nagel, And J. Shanken (2010): "A Skeptical Appraisal of Asset-Pricing Tests," Journal of Financial Economics, 96, 175-194.

LÖNN, S. O. R. (2018): "Optimizing large portfolios using risk factors and sparse hedging," Maastricht University Working Papers.

LÖnn, S. O. R. And P. C. Sсhотman (2018): "Empirical asset pricing with many assets and short time series," Maastricht University Working Papers.

Mazumder, R. And T. Hastie (2012): "The graphical lasso: New insights and alternatives," Electronic journal of statistics, 6, 2125.

Meinshausen, N. And P. Bühlmann (2006): "High-dimensional graphs and variable selection with the lasso," The annals of statistics, 34, 1436-1462.

Meurant, G. (1992): "A review on the inverse of symmetric tridiagonal and block tridiagonal matrices," SIAM Journal on Matrix Analysis and Applications, 13, 707-728.

Michaud, R. (1989): "The Markowitz optimization enigma: Is optimized optimal?" Financial Analysts Journal, 45, 31-âĂŞ42.

Pastor, L. and R. Stambaugh (2003): "Liquidity Risk and Expected Stock Returns," Journal of Political Economy, 111, 642-685.

Ravikumar, P., M. J. Wainwright, G. Raskutti, and B. Yu (2011): "High-dimensional covariance estimation by minimizing âĎş1-penalized logdeterminant divergence," Electronic Journal of Statistics, 5, 935-980.

Ross, S. (1976): "The Arbitrage Theory of Capital Asset Pricing," Journal of Economic Theory, 13, 341-360.

Rothman, A. J., P. J. Bickel, E. Levina, And J. Zhu (2008): "Sparse permutation invariant covariance estimation," Electronic Journal of Statistics, $2,494-515$.

SCHERER, B. (2011): "A note on the returns from minimum variance investing," Journal of Empirical Finance, 18, 652-660.

Sharpe, W. (1964): "Capital Asset Prices: A Theory for Market Equilibrium under Conditions of Risk," Journal of Finance, 19, 425-442. 
Shiller, R. J. (1981): "Do stock prices move too much to be justified by subsequent chages in dividends?" American Economic Review, 71, 421-436.

Stevens, G. V. (1998): "On the inverse of the covariance matrix in portfolio analysis," The Journal of Finance, 53, 1821-1827.

Subrahmanyam, A. (1991): "A theory of trading in stock index futures," The Review of Financial Studies, 4, 17-51.

Tibshirani, R. (1996): "Regression shrinkage and selection via the lasso," Journal of the Royal Statistical Society: Series B (Methodological), 58, 267-288.

Vassalou, M. (2003): "News Related to Future GDP Growth as a Risk Factor in Equity Returns," Journal of Financial Economics, 68, 43-74.

VAYANOS, D. AND J. WANG (2011): "Liquidity and asset returns under asymmetric information and imperfect competition," The Review of Financial Studies, $25,1339-1365$.

Wainwright, M. J. (2009): "Sharp thresholds for High-Dimensional and noisy sparsity recovery using $\ell_{1}$-Constrained Quadratic Programming (Lasso)," IEEE transactions on information theory, 55, 2183-2202.

YUAN, M. AND Y. LiN (2007): "Model selection and estimation in the Gaussian graphical model," Biometrika, 94, 19-35.

ZhaO, P. AND B. YU (2006): "On model selection consistency of Lasso," Journal of Machine learning research, 7, 2541-2563.

Zou, H. AND T. HASTIE (2005): "Regularization and variable selection via the elastic net," Journal of the Royal Statistical Society: Series B (Statistical Methodology), 67, 301-320. 



\section{Nederlandse Samenvatting}

"We judge economics by what it can produce. As such, economics is rather more like engineering than physics: more practical than spiritual."

- Robert J. Shiller (1946-) 
Dit proefschrift onderzoekt het gebruik van empirische econometrie in de disciplines asset pricing en portefeuillemanagement. De voornaamste uitdaging in deze scriptie omvat de econometrische behandeling van data met zeer grote crosssectionele dimensies. Met andere woorden, hoe men op empirische wijze financiële vragen beantwoordt wanneer bijvoorbeeld het aantal aandelen of voorspellers erg hoog is. Deze uitdaging is relevant in menig macro-economische applicaties, maar kent voornamelijk een grote hoeveelheid toepassingen in het gebied van financiën. De reden hiervoor is de immense cross-sectionele omvang van financiële markten. Vanuit een economisch oogpunt is de schatting en behandeling van financieel risico de rode draad die alle hoofdstukken met elkaar verbindt. Dit gemeenschappelijke onderwerp neemt verschillende vormen aan in de hoofdstukken; van vragen omtrent de beste manier om rendementsverschillen te modelleren tot het combineren van aandelen in een portefeuille met minimale volatiliteit. Traditionele econometrische methoden schieten vaak tekort of zijn zelfs ontoepasbaar wanneer deze vragen worden gesteld in de context van een grote hoeveelheid aandelen. Een uitbreiding van de bestaande toolkit is noodzakelijk in de meeste gevallen. Om deze uitdagingen het hoofd te bieden, maak ik gebruik van gerelateerde onderwerpen in de disciplines machinaal leren en hoog-dimensionele statistiek. Het blind toepassen van deze technieken is echter onvoldoende om voortgang te bewerkstelligen op economisch vlak. Voor een empirische benadering van asset pricing en portefeuillemanagement is in de meeste gevallen een doordachte combinatie van logische economische restricties en efficiënte regularisatie noodzakelijk.

In hoofdstuk 2 en 4 staan empirische asset pricing en risico verbonden aan macro-economische en financiële liquiditeit centraal. Hoofdstuk 2 ontwikkelt een methodiek om macro-economische risico modellen te beoordelen. De bijdrage van deze vernieuwde methodologie is dat deze geldig blijft zelfs wanneer het aantal aandelen veel harder groeit in relatie tot de lengte van de tijdreeksen. Tevens presteren de methoden beter dan alternatieven in de literatuur. Onze aanpak laat zien dat in een vergelijking van macro-economische risico factoren, de relatieve bijdrage van de meest klassieke factor, bijvoorbeeld consumptie groei, zeer groot is. Voorts vinden wij dat de relatieve toename van het voorspellende onderscheidingsvermogen (power) van deze factoren in grote mate toeneemt als de modellen niet-lineaire risico blootstellingen in acht nemen. Dit suggereert dat de functionele vorm van macro-economische modellen een grotere restrictieve component is vergeleken met (verwachte uitkomsten voor) financiële risico modellen. Dit kan 
gedeeltelijk de discrepantie in statistische fit verklaren die doorgaans aanwezig is wanneer deze twee klassen modellen met elkaar vergeleken worden.

Hoofdstuk 2 bestaat uit een algemene methodiek voor het beoordelen en vergelijken van modellen. Hoofdstuk 4 verlegt de focus naar een specifieke bron van risico die veroorzaakt wordt door financiële illiquiditeit. In de financiële zin refereert het woord illiquiditeit aan de eenvoud waarmee een aandeel verhandeld kan worden. Dit concept is empirisch niet eenduidig en bestaat uit een veelvoud aan facetten. Wij nemen het perspectief van prijs sensitiviteit en weerstand tegen handelsstromen. Empirische financiële toepassingen worden vaak benaderd als een probleem waarin de dimensies van markten worden gereduceerd tot een kleinere set van eigenschappen. De vorm van financiële liquiditeit die bestudeerd wordt in dit hoofdstuk staat een zodanige dimensie reductie niet toe. Dit leidt tot een set van overlappende orderstromen, zijnde onafhankelijke variabelen, die veel groter is dan de hoeveelheid beschikbare data. Wij stellen een oplossing voor waarbij prijs sensitiviteit geschat wordt door statistische regularisatie in de vorm van een elastisch net. Onze resultaten geven aan dat deze aanpak informatie extraheert die helpt rendementsafwijkingen te verklaren, in het bijzonder voor aandelen met zeer lage marktkapitalisatie.

Mijn derde hoofdstuk verlegt het perspectief van asset pricing naar portefeuille optimalisatie. In dit hoofdstuk introduceer ik een aanpak voor minimum-variantie optimalisatie die statistische regularisatie en het klassieke lineaire modelleren combineert. Meer gedetailleerd: de voorgestelde methode bestaat uit een combinatie van standaard financiële risico modellen en een hoog-dimensionele grafische selectie van modellen. Vanuit een financieel oogpunt combineert deze aanpak de lineaire schatting van gemeenschappelijke blootstellingen en voorwaardelijke schatting van sparse hedging portefeuilles. Intuitief is de regularisatie noodzakelijk om de tekortkomingen van de prominente financiële factoren te compenseren. Een bijkomend positief bijproduct is dat deze methode enkele van de empirisch meest problematische aspecten van portefeuillemanagement omzeilt. De bevindingen in deze scriptie wijzen uit dat de voorgestelde aanpak empirisch beter presteert dan de meest vooraanstaande alternatieven in het gebied van portefeuille risico reductie.

In de samenvatting van mijn proefschrift wil ik benadrukken dat het belangrijk is om economische en statistische restricties samen toe te passen in de context van hoog-dimensionele economische en financiële problemen. Het is niet relevant of de bron van de hoog-dimensionele uitdaging een complexe functionele vorm of een grote set beschikbare voorspellers is, want de combinatie van statistische en 
economische restricties heeft wederzijdse voordelen. De bevindingen in deze scriptie geven aan dat er een groot potentieel is in macro-economische risico modellen wanneer deze ook in staat zijn om niet-lineaire relaties in acht te nemen. Tevens kan er grote vooruitgang worden geboekt in het gebied van portefeuillemanagement door op-maat-gemaakte statistische regularisatie. Deze dient dan in staat te zijn om bekende financiële verschijnselen, zoals bijvoorbeeld de aanwezigheid vaan gemeenschappelijkheden, te accommoderen. 


\section{Curriculum Vitae}

"Deyr fé, deyja frcendur, deyr sjálfur ið sama; ek veit einn at aldri deyr dómr um dauðan hvern.”

- Hávamál 
Rasmus Lönn was born on December 6, 1989, in Uddevalla, Sweden. He graduated high school in 2008 at Uddevalla Gymnasieskola having completed the engineering track. That same year he enrolled at Gotheburg University were he earned a BSc in Economics and a BSc in Statistics. Rasmus continued his university education at Maastricht University where he also worked as a research assistant before completing his MSc degree in Economic and Financial research in 2015.

In 2015, upon graduation from the research master he started the working as a Ph.D. candidate at the Department of Quantitative Economics, under the supervised of Prof.dr. Peter Schotman and Prof.dr. Jean-Pierre Urbain. Later, supervision came to include Dr. Nalan Basturk and Dr. Lenard Lieb. This thesis presents the findings of this research. In 2017 Rasmus went to work as a Ph.D. intern at the Bank of England in the Statistics \& Regulatory data division. Rasmus has presented his research at many international conferences including 2nd International Russia Longitudinal Monitoring Survey of HSE User Conference in Moskow 2015, Conference on The Econometrics of Financial Markets in Stockholm 2017, Tenth Annual Society of Financial Econometrics in New York 2017, Workshop on Financial Econometrics 2018 in Örebro and Twelfth Annual Society of Financial Econometrics in Shanghai 2019. In September 2019 he joined the Econometric Institute of Erasmus University Rotterdam on a tenure track position. 